\title{
Analytical and Computational Study of Flame Acceleration in Tubes: Effect of Wall Friction
}

\author{
Berk Demirgok \\ West Virginia University
}

Follow this and additional works at: https://researchrepository.wvu.edu/etd

\section{Recommended Citation}

Demirgok, Berk, "Analytical and Computational Study of Flame Acceleration in Tubes: Effect of Wall Friction" (2013). Graduate Theses, Dissertations, and Problem Reports. 301.

https://researchrepository.wvu.edu/etd/301

This Thesis is protected by copyright and/or related rights. It has been brought to you by the The Research Repository @ WVU with permission from the rights-holder(s). You are free to use this Thesis in any way that is permitted by the copyright and related rights legislation that applies to your use. For other uses you must obtain permission from the rights-holder(s) directly, unless additional rights are indicated by a Creative Commons license in the record and/ or on the work itself. This Thesis has been accepted for inclusion in WVU Graduate Theses, Dissertations, and Problem Reports collection by an authorized administrator of The Research Repository @ WVU. For more information, please contact researchrepository@mail.wvu.edu. 


\section{Analytical and Computational Study of Flame Acceleration in Tubes: \\ Effect of Wall Friction \\ Berk Demirgok}

Thesis submitted to the

Benjamin M. Statler College of Engineering and Mineral Resources

at

West Virginia University

in Partial Fulfillment of the Requirements for the Degree of

Master of Science

in

Mechanical Engineering

\section{V'yacheslav Akkerman, Ph.D., Chair \\ Ismail B. Celik, Ph.D.}

Halin Li, Ph.D.

Scott W. Wayne, Ph.D.

Department of Mechanical and Aerospace Engineering

Morgantown, West Virginia

(2013)

Keywords: combustion, theory, modeling, flame acceleration, tubes, channels

Copyright 2013 Berk Demirgok 


\section{ABSTRACT \\ Analytical and Computational Study of Flame Acceleration in Tubes: \\ Effect of Wall Friction \\ Berk Demirgok}

Premixed flame acceleration is especially strong in the case of flame propagation in tubes or channels. Being a reasonably simple configuration to investigate fundamental flame properties, combustion tubes have numerous practical applications such as safety issues in mines, subways and power plants. This work is devoted to the analytical formulation and computational simulations of premixed flame acceleration induced by wall friction in tubes/channels. Specifically, the evolution of the flame dynamics and morphology is determined, and the main characteristics of the flame acceleration such as the flame shape and propagation speed, the acceleration rate as well as the combustion-generated velocity profile in the fresh premixture are quantified. It is shown that the flame acceleration is promoted with the increase in the thermal expansion in the burning process, whereas it weakens with the increase in the Reynolds number. The intrinsic accuracy and the limitations of the analytical theory are determined and validated by means of direct numerical simulations. Computational and analytical results are compared with recent experiments, and the numerical simulations bridge a certain gap between the experimental measurements and analytical formulations. 


\section{Acknowledgements}

I would like to express my gratitude to several key persons who have contributed towards the completion of this thesis and have influenced my professional development as a researcher.

First and foremost, I would like to thank Dr. V'yacheslav Akkerman, who initially gave me the opportunity to pursue my educational career with WVU, and continuously provided me with his huge support and guidance throughout my time here. I sincerely thank my committee members, Dr. Ismail B. Celik, Dr. Hailin Li and Dr. Scott W. Wayne for their valuable time and suggestions. I am also very grateful to my friends and lab partners for useful discussions.

Also, I would like to acknowledge Dr. Damir Valiev of Sandia National Laboratories, Livermore, CA. The computational component of this thesis would not be possible without his comprehensive support and consulting.

Besides, I am thankful to Dr. Ming-Hsun Wu of Taiwan National Cheng Kung University, who provided us with his experimental data.

Finally, I would like to mention my family that never questioned my ability of obtaining an advanced degree and that has stuck by my side throughout. 
I dedicate this thesis to my parents Bulent Demirgok and Zahide Demirgok. 


\section{Table of Contents}

$\begin{array}{lllllllllllll}\text { Abstract... } & \ldots & \ldots & \ldots & \ldots & \ldots & \ldots & \ldots & \ldots & \ldots & \ldots & \ldots & \text { ii }\end{array}$

$\begin{array}{lllllllllllll}\text { Acknowledgments... } & \ldots & \ldots & \ldots & \ldots & \ldots & \ldots & \ldots & \ldots & \ldots & \ldots & \text { iii }\end{array}$

$\begin{array}{llllllllllllll}\text { Dedication... } & \ldots & \ldots & \ldots & \ldots & \ldots & \ldots & \ldots & \ldots & \ldots & \ldots & \ldots & \text { iv }\end{array}$

$\begin{array}{llllllllllllll}\text { Table of Contents... } & \ldots & \ldots & \ldots & \ldots & \ldots & \ldots & \ldots & \ldots & \ldots & \ldots & \mathrm{V}\end{array}$

$\begin{array}{lllllllllllll}\text { Nomenclature... } & \ldots & \ldots & \ldots & \ldots & \ldots & \ldots & \ldots & \ldots & \ldots & \ldots & \text { vii }\end{array}$

$\begin{array}{lllllllllllll}\text { List } \text { of Figures... } & \ldots & \ldots & \ldots & \ldots & \ldots & \ldots & \ldots & \ldots & \ldots & \ldots & \text { xi }\end{array}$

$\begin{array}{lllllllllllll}\text { Chapter 1: Introduction... } & \ldots & \ldots & \ldots & \ldots & \ldots & \ldots & \ldots & \ldots & 1\end{array}$

1.1. Fundamentals of Combustion...

$\begin{array}{lllllllllll}\text { 1.2. Overview and Objectives... } & \ldots & \ldots & \ldots & \ldots & \ldots & \ldots & \ldots & 5\end{array}$

$\begin{array}{llllllllllll}\text { 1.2.1. Overview... } & \ldots & \ldots & \ldots & \ldots & \ldots & \ldots & \ldots & \ldots & \ldots & 5\end{array}$

$\begin{array}{llllllllllll}\text { 1.2.2. Objectives... } & \ldots & \ldots & \ldots & \ldots & \ldots & \ldots & \ldots & \ldots & \ldots & 9\end{array}$

\section{Chapter 2: Theory of Flame Acceleration in Tubes due to Wall Friction:}

$\begin{array}{llllllll}\text { Intrinsic Limitations and Accuracy } \ldots & \ldots & \ldots & \ldots & \ldots & \ldots & \ldots & 10\end{array}$

2.1. Basics of the Bychkov-Akkerman Formulation... $\ldots \begin{array}{llllllll} & \ldots & \ldots & \ldots & \ldots & 10\end{array}$

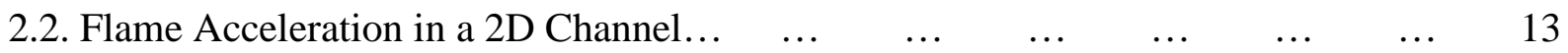

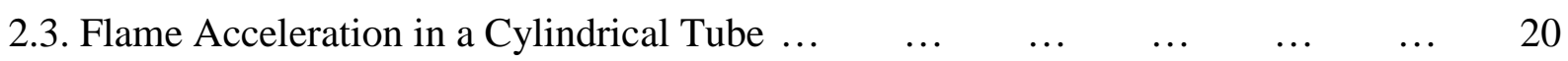

$\begin{array}{llllllllllll}\text { 2.4. Conclusions... } & \ldots & \ldots & \ldots & \ldots & \ldots & \ldots & \ldots & \ldots & \ldots & 28\end{array}$

$\begin{array}{lllllllll}\text { Chapter 3: Computational Platform... } & \ldots & \ldots & \ldots & \ldots & \ldots & \ldots & 29\end{array}$

$\begin{array}{llllllllllll}\text { 3.1. Governing Equations... } & \ldots & \ldots & \ldots & \ldots & \ldots & \ldots & \ldots & \ldots & 29\end{array}$

$\begin{array}{lllllllllll}\text { 3.2. Numerical Scheme... } & \ldots & \ldots & \ldots & \ldots & \ldots & \ldots & \ldots & \ldots & 34\end{array}$

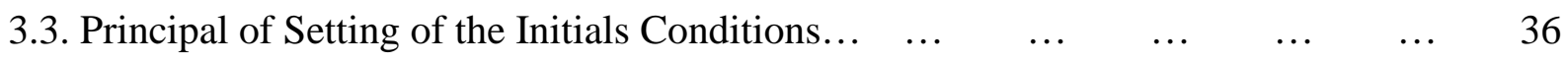


Chapter 4: Effect of Thermal Expansion on Flame Propagation in Channels $\begin{array}{lllll}\text { with Nonslip Walls: Numerical and Analytical Consideration... } & \ldots & \ldots & 38\end{array}$

$\begin{array}{lllllllllll}\text { 4.1. Motivation... } & \ldots & \ldots & \ldots & \ldots & \ldots & \ldots & \ldots & \ldots & \ldots & 38\end{array}$

$\begin{array}{llllllll}\text { 4.2. Computational Results and Discussion... } & \ldots & \ldots & \ldots & \ldots & \ldots & \ldots & 39\end{array}$

$\begin{array}{lllllllllll}\text { 4.3. Conclusions... } & \ldots & \ldots & \ldots & \ldots & \ldots & \ldots & \ldots & \ldots & \ldots & 52\end{array}$

Chapter 5: Analysis of Ethylene-Oxygen Combustion in Micro-Pipes... $\quad \ldots \quad 53$

$\begin{array}{lllllllllll}\text { 5.1. Formulation... } & \ldots & \ldots & \ldots & \ldots & \ldots & \ldots & \ldots & \ldots & \ldots & 53\end{array}$

$\begin{array}{lllllllllll}\text { 5.2. Experiments... } & \ldots & \ldots & \ldots & \ldots & \ldots & \ldots & \ldots & \ldots & \ldots & 55\end{array}$

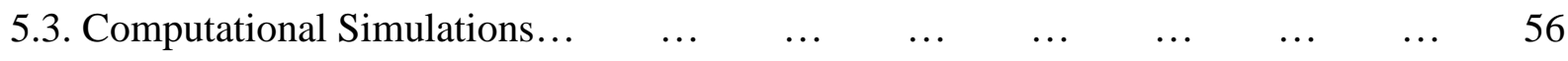

$\begin{array}{llllllllll}\text { 5.4. Results and Discussion... } & \ldots & \ldots & \ldots & \ldots & \ldots & \ldots & \ldots & \ldots & 57\end{array}$

$\begin{array}{lllllllllll}\text { 5.5. Conclusions... } & \ldots & \ldots & \ldots & \ldots & \ldots & \ldots & \ldots & \ldots & \ldots & 61\end{array}$

$\begin{array}{lllllllllll}\text { Chapter 6: Summary } & \ldots & \ldots & \ldots & \ldots & \ldots & \ldots & \ldots & \ldots & \ldots & 62\end{array}$

$\begin{array}{lllllllllllll}\text { References... } & \ldots & \ldots & \ldots & \ldots & \ldots & \ldots & \ldots & \ldots & \ldots & \ldots & \ldots & 63\end{array}$

$\begin{array}{llllllllllll}\text { APPENDIX A.. } & \ldots & \ldots & \ldots & \ldots & \ldots & \ldots & \ldots & \ldots & \ldots & \ldots & 65\end{array}$

$\begin{array}{llllllllllll}\text { APPENDIX В... } & \ldots & \ldots & \ldots & \ldots & \ldots & \ldots & \ldots & \ldots & \ldots & \ldots & 75\end{array}$

$\begin{array}{llllllllllll}\text { APPENDIX C... } & \ldots & \ldots & \ldots & \ldots & \ldots & \ldots & \ldots & \ldots & \ldots & \ldots & 76\end{array}$ 


\section{Nomenclature}

\section{Letters}

A

$\mathrm{C}_{\mathrm{p}}$

$[\mathrm{J} / \mathrm{Kg}]$

CPU

DL

DNS

$\mathrm{D}_{\text {th }}$

$E_{a}$

$\mathrm{L}_{\mathrm{f}}$

$\mathrm{Ma}$

$\mathrm{m}_{\mathrm{b}}$

$\mathrm{m}_{\mathrm{f}}$

$\mathrm{n}$

$\mathrm{P}$

Pr

Q

$\mathrm{q}_{\mathrm{i}}$

$\mathrm{R}$

RAM

$\operatorname{Re}$

$\mathrm{R}_{\mathrm{p}}$

$\mathrm{S}_{2 \mathrm{D}}$
[Pa]

$[\mathrm{J}]$

[mm]

kj/mol]

[m]

[g/mol]

[g/mol]

$\mathrm{mm}]$

Reaction rate

Heat capacity

Central processing unit

Darieus- Landau

Direct numerical simulations

Thermal diffusivity

Activation energy

Flame front thickness

Mach number

molecular weight of the burnt matter

molecular weight of the fuel mixture

normal vector

Pressure

Prandtl number

Energy release from the reaction

energy diffusion vector component

Radius of a tube

Random memory access

Reynolds number

Universal gas constant

Total length of the flame front in a 2D 
geometry

$\begin{array}{lll}\mathrm{S}_{\mathrm{cyl}} & {[\mathrm{m}]} & \begin{array}{l}\text { Total length of the flame front in an } \\ \text { axisymmetric geometry }\end{array} \\ \mathrm{Sc} & {\left[\mathrm{m}^{2}\right]} & \begin{array}{l}\text { Schmidt number } \\ \mathrm{S}_{\mathrm{f}}\end{array} \\ \mathrm{S}_{\mathrm{w}} & {\left[\mathrm{m}^{2}\right]} & \text { The area of the plane front } \\ \mathrm{T} & {[\mathrm{K}]} & \text { Temperature } \\ \mathrm{T}_{\mathrm{b}} & {[\mathrm{K}]} & \text { Temperature of the burnt matter } \\ \mathrm{U}_{\mathrm{f}} & {[\mathrm{cm} / \mathrm{s}]} & \text { Unstretched laminar (planar) flame } \\ & & \text { speed } \\ \mathrm{U}_{\mathrm{tip}} & {[\mathrm{cm} / \mathrm{s}]} & \text { Flame tip velocity } \\ \mathrm{U}_{\mathrm{w}} & {[\mathrm{cm} / \mathrm{s}]} & \text { Curved flame velocity } \\ \mathrm{u}_{\mathrm{z}} & & \text { Flow velocity related to burning rate } \\ \mathrm{WVU} & & \text { West Virginia University }\end{array}$

\section{Greek Symbols}

$\Omega_{\mathrm{W}}$

$\sigma$

$\sigma_{\text {numerical }}$

$\sigma_{\text {analytical }}$

$\sigma_{0}$

$\sigma_{1}$
Scaled flame velocity

Dimensionless acceleration rate

Dimensionless acceleration rate of the numerical approach

Dimensionless acceleration rate of the analytical approach

Dimensionless acceleration rate in the $0^{\text {th }}$ order approximation

Dimensionless acceleration rate in 
the $1^{\text {st }}$ order approximation

$\zeta_{\mathrm{ij}}$

$\tau$

$\tau_{\mathrm{R}}$

$v$

$\mu$

$\xi_{\text {tip }}$

$\rho_{\mathrm{b}}$

$\rho_{\mathrm{f}}$

$\pi$

$\Omega_{\mathrm{ss}}$

$\Theta$

$\kappa$
Stress tensor

Scaled time

Characteristic time

[kg/s.m]

[kg/s.m]

Dynamic viscosity

Scaled flame tip position

Density of the burnt matter

Density of the fuel

Pi number

Parameter characterizing the total burning rate in the isobaric limit

Thermal expansion coefficient

Thermal conduction coefficient 


\section{List of Figures}

FIG. 1.1. Typical internal structure of a planar flame front (a), with the characteristic temperature and density distribution inside it (b), as well as with the profiles of the scaled temperature $T / T_{b}$, the local mass fraction of the fresh gas $Y$ and the reaction rate $A$ inside the burning zone (c) $\quad \ldots \quad 2$ FIG. 1.2. Evolution of a finger flame front..... FIG. 1.3 Wall friction (Shelkin) scenario of flame acceleration in smooth tubes... $\ldots \quad \ldots \quad \ldots \quad 7$

FIG. 1.4. Schematic of the physical mechanism of flame acceleration in obstructed tube $\quad \ldots \quad 8$

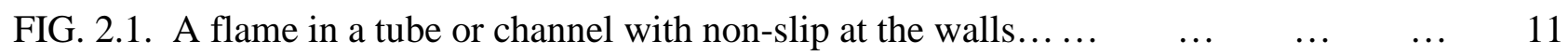
FIG. 2.2. The flame acceleration rate $\sigma$ versus the flame propagation Reynolds number Re at the fixed thermal expansion coefficients $\Theta=4 ; 6 ; 8 ; 10$. Equations (2.8) and (2.10) are shown by $\begin{array}{lllllllll}\text { dashed and solid lines, respectively } \ldots & \ldots & \ldots & \ldots & \ldots & \ldots & \ldots & \ldots & 16\end{array}$ FIG. 2.3. The flame acceleration rate $\sigma$ versus the thermal expansion coefficient $\Theta$ at the fixed flame propagation Reynolds numbers $\operatorname{Re}=5 ; 10 ; 20$. Equations (2.8) and (2.10) are shown by $\begin{array}{lllllllll}\text { dashed and solid lines, respectively... } & \ldots & \ldots & \ldots & \ldots & \ldots & \ldots & \ldots & 17\end{array}$

FIG. 2.4. Relative error (in \%) versus thermal expansion coefficient $\Theta$ at fixed propagation Reynolds numbers such as $\mathrm{Re}=20,10$ and 5 shown by dotted, dashed and solid lines, respectively (a); and the relative error percentage versus propagation Reynolds number at fixed thermal expansion such as $\Theta=10,8,6$ and 4 shown by solid \&dotted, dashed, dotted and solid lines, $\begin{array}{lllllllllllll}\text { respectively }(\mathrm{b}) \ldots & \ldots & \ldots & \ldots & \ldots & \ldots & \ldots & \ldots & \ldots & \ldots & \ldots & 19\end{array}$ FIG. 2.5. Contour scheme (the error isolines (in \%)) demonstration of Propagation Reynolds $\begin{array}{lllllllll}\text { number versus thermal expansion } \Theta \ldots & \ldots & \ldots & \ldots & \ldots & \ldots & \ldots & \ldots & 20\end{array}$ FIG. 2.6. $\sigma$ versus $\operatorname{Re}$ at $\Theta=4 ; 6$; 8 (a), and versus $\Theta$ at $\operatorname{Re}=5$; 10; 20 (b). Equations (2.15) and $\begin{array}{llllllll}(2.16) & \text { are shown by dashed and solid lines, respectively... } & \ldots & \ldots & \ldots & \ldots & \ldots & 22\end{array}$ FIG. 2.7. The relative error (in \%), Eq. (2.11), versus $\Theta$ at $R e=5,10$, 20, shown by solid, dashed and dotted lines, respectively (a); and versus Re at $\Theta=3,4,6$ shown by solid, dashed and dotted $\begin{array}{lllllllllll}\text { lines, respectively }(\mathrm{b}) \ldots & \ldots & \ldots & \ldots & \ldots & \ldots & \ldots & \ldots & \ldots & \ldots & 23\end{array}$ FIG. 2.8. Contour scheme (the error isolines (in \%)) demonstration in the Re- $\Theta$ diagram $\quad \ldots \quad 24$ FIG. 2.9. $\sigma$ versus Re at $\Theta=4$; 6; 8 (a); and versus $\Theta$ at $\operatorname{Re}=5$; 10; 20 (b). Equations (2.15) and (2.17) are $\begin{array}{lllllllll}\text { shown by dashed and solid lines, respectively } & \ldots & \ldots & \ldots & \ldots & \ldots & \ldots & \ldots & 25\end{array}$ 
FIG. 2.10. The relative error (in \%), Eq. (2.11), versus $\Theta$ at $\operatorname{Re}=5,10,20$, shown by solid, dashed and dotted lines, respectively (a); and versus Re at $\Theta=3,4,6$ shown by solid, dashed and dotted

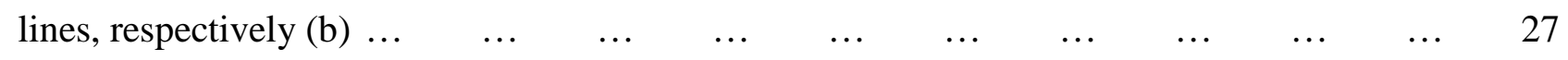
FIG. 2.11. Contour scheme (the error isolines (in \%)) demonstration in the $\operatorname{Re}-\Theta$ diagram ... 28 FIG. 3.1. The sketch of the grid with variable resolution used in numerical simulations $\quad \ldots \quad \ldots \quad 32$ FIG. 3.2. Profiles of the scaled temperature $T / T \mathrm{~b}$, the mass fraction $Y$, and the reaction rate... $\quad \ldots$

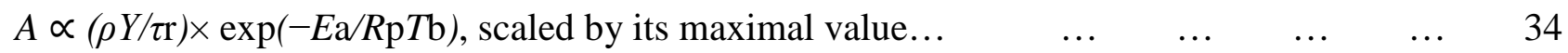
FIG. 4.1. The scaled flame tip position $\xi_{\text {tip }}$ versus the scaled time $\tau$ at fixed thermal expansion coefficients, namely $\Theta=12(\mathrm{a}), \Theta=10$ (b), $\Theta=9$ (c), and $\Theta=8$ (d), with various flame propagation Reynolds numbers in each plot and the scaled flame tip position $\xi_{\text {tip }}$ versus the scaled time $\tau$ at thermal expansion coefficients $\Theta$ $=12,10,8$ and 6 and fixed propagation Reynolds number $\operatorname{Re}=20$ (e) $\operatorname{Re}=10$ (f) $\ldots \quad \ldots \quad \ldots \quad 43$

FIG. 4.2. The flame acceleration rate $\sigma$ versus propagation Reynolds number Re at thermal expansion $\Theta=12$

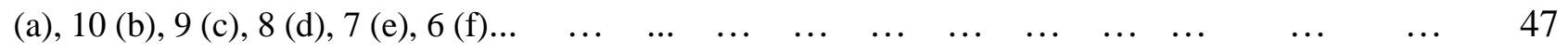
FIG. 4.3. The scaled flame tip position $\xi_{\text {tip }}$ versus the scaled time $\tau$ at fixed thermal expansion coefficient $\Theta=$ 5 and flame propagation Reynolds numbers $R e=30$ (a). The scaled flame tip velocity $U_{\text {tip }} / U_{\mathrm{f}}$ versus the scaled time $\tau$ at fixed thermal expansion coefficient $\Theta=5$ and flame propagation Reynolds numbers $\operatorname{Re}=30$ (b). The scaled flame tip position $\xi_{\text {tip }}$ versus the scaled time $\tau$ at fixed thermal expansion coefficient $\Theta=4$ and flame propagation Reynolds numbers $R e=40$ (c). The scaled flame tip velocity $U_{\text {tip }} / U_{f}$ versus the scaled time $\tau$ at fixed thermal expansion coefficient $\Theta=4$ and flame propagation Reynolds numbers $\operatorname{Re}=40$ (d)

FIG. 4.4. Evolution of the flame shape for a small thermal expansion, $\Theta=4$ Fig. 4.3 (a-c) as well as for the

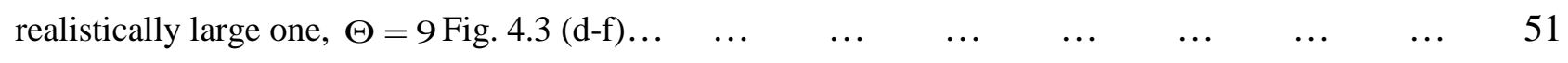
FIG. 5.1. Evolution of the flame tip in a tube of radii $\mathrm{R}=0.25 \mathrm{~mm}(\mathrm{a})$ and $0.5 \mathrm{~mm}(\mathrm{~b}) \ldots \quad \ldots \quad \ldots \quad 58$ FIG. 5.2. Evolution of the flame tip in a tube of $\mathrm{R}=0.25 \mathrm{~mm}$ for $\Omega_{\mathrm{ss}}=4.2$. The cases of the acceleration rate given by Eq. (2.17), $\sigma_{1}$ and Eq. (2.16), $\sigma_{0}$, are shown by the dashed and solid lines, respectively. Filled rectangles show the experimental results while dotted line represents the $\begin{array}{lllllllllllllll}\text { DNS ones... } & \ldots & \ldots & \ldots & \ldots & \ldots & \ldots & \ldots & \ldots & \ldots & \ldots & \ldots & \ldots & 59\end{array}$ FIG. 5.3. Evolution of the flame tip in a tube of $\mathrm{R}=0.5 \mathrm{~mm}$ for $\Omega_{\mathrm{ss}}=4.2$. The cases of the acceleration rate given by Eq. (2.17), $\sigma_{1}$, and Eq. (2.16), $\sigma_{0}$, are shown by the dashed and solid 
lines, respectively. Filled rectangles show the experimental results while dotted line represents the $\begin{array}{lllllllllllllll}\text { DNS ones... } & \ldots & \ldots & \ldots & \ldots & \ldots & \ldots & \ldots & \ldots & \ldots & \ldots & \ldots & \ldots & 59\end{array}$

FIG. 5.4. Evolution of the flame tip in a tube of $\mathrm{R}=0.5 \mathrm{~mm}$ for $\Omega_{\mathrm{ss}}=7.1$. The cases of the acceleration rate given by Eq. (2.16), $\sigma_{1}$, and Eq. (5.16), $\sigma_{0}$, are shown by the dashed and solid lines, respectively. Filled rectangles show the experimental results while dotted line represents the DNS ones ... $\quad \ldots \quad \ldots \quad \ldots 0$ FIG. 5.5: Evolution of the flame shape in a tube of radius $R=56 \mathrm{~L}_{\mathrm{f}}=0.25 \mathrm{~mm}$. The colors represent the temperature from $300 \mathrm{~K}$ in the fuel mixture (blue) to the corresponding burnt matter temperature (red)... 61 


\section{Chapter 1: Introduction}

\subsection{Fundamentals of Combustion}

What is Combustion? While it can generally be defined as an exothermal chemical reaction, such a definition incorporates a great variety of different processes, starting with standard oxidation of coal, oil, natural gas, alcohol and hydro-carbon fuels, and ending with astrophysical applications like thermonuclear reactions in Supernovae. This thesis is limited to burning of premixed air-fuel mixture, when all chemical components, necessary for the reaction, are present in a air-fuel mixture from the very beginning. In that case, if the heat release in the burning process exceeds the thermal losses, then the reaction is self-supporting, and once ignited, the reaction typically spreads through the gas as a rather thin, well-localized front until all the fuel is burned. Such a scenario is typically observed in car engines, subways, mines or various laboratory setups.

Basically, two main self-supporting regimes of combustion are distinguished: a flame (also known as deflagration) and a detonation (Bychkov and Liberman, 2000). In the case of a flame, the reaction propagates due to thermal conduction, transporting energy from the hot burnt matter to the cold fuel-air premixture (hereafter: fuel mixture). In a detonation, the process occurs due to shock waves, which compress the air-fuel mixture to higher temperature. Consequently, the flame is a subsonic burning regime, which propagates 2-4 orders of magnitude slower than the fast (supersonic) detonation. The present study is focused mostly on the flame propagation.

So, what is a flame in fluid environment? It can be described as a typical reacting flow consisting of the regions of the unburned fuel mixture (where the reaction has not begun yet), the burnt matter (where the reaction is completed), and a thin zone called a "flame front" separating them. The inner structure of a planar flame front (which is the simplest to study) is shown 
schematically in Fig. 1.1a, while the characteristic density/temperature distributions in such a system are illustrated in Fig. 1.1b.

(a)
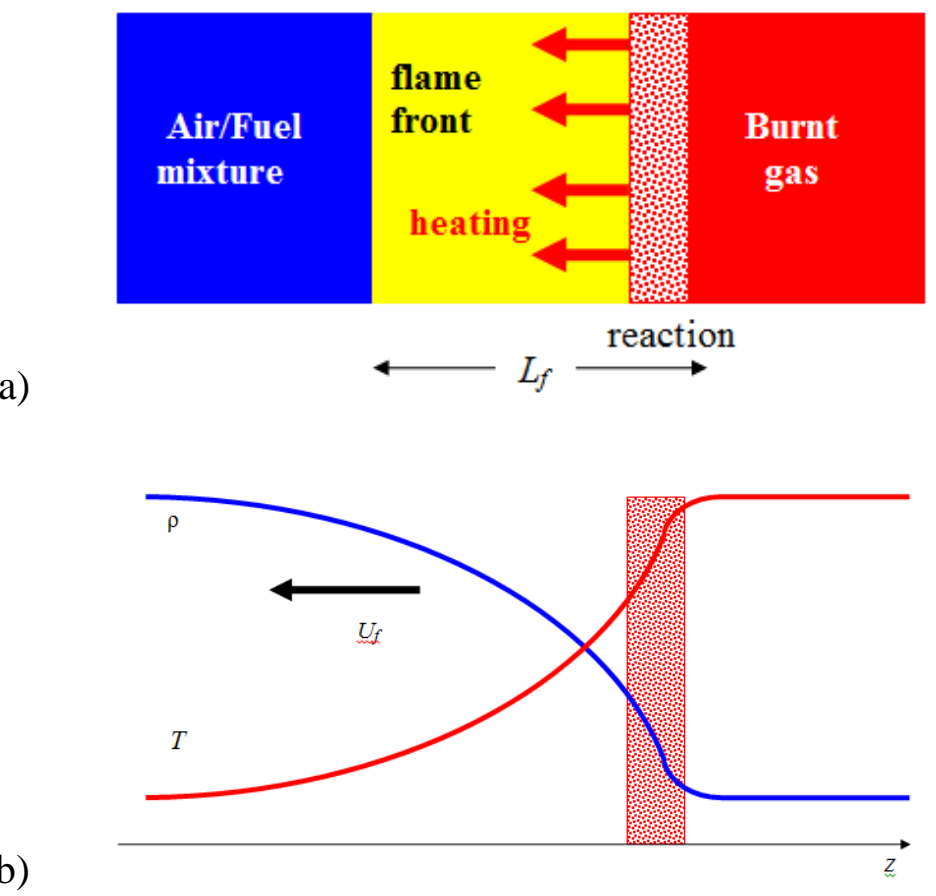

(b)

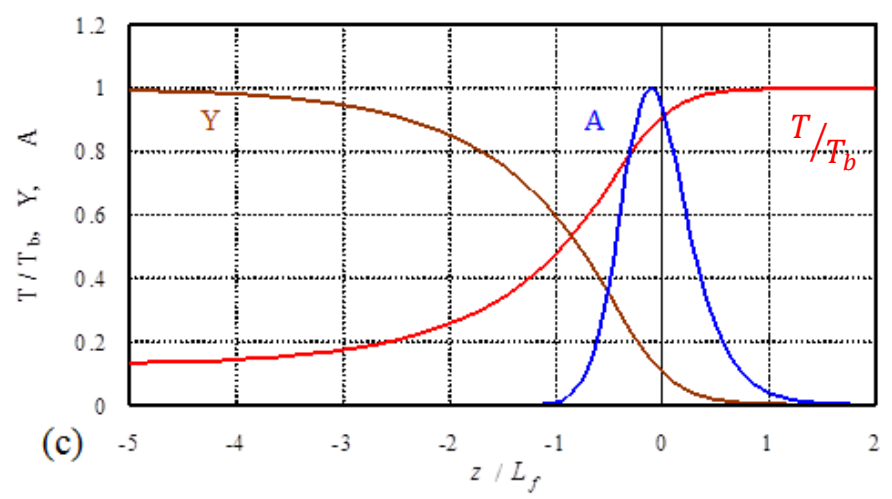

FIG. 1.1. Typical internal structure of a planar flame front (a), with the characteristic temperature and density distribution inside it (b), as well as with the profiles of the scaled temperature $T / T_{b}$, the local mass fraction of the fresh gas $Y$ and the reaction rate $A$ inside the burning zone (c) 
It is well known that burning does not occur at room temperature without forced ignition, while at high temperatures the reaction goes very fast. This is because of strong temperaturedependence of the reaction rate of any burning process. Indeed, a twice increase in the fuel temperature sometimes amplifies the reaction rate $10^{8} \sim 10^{9}$ times. (Bychkov and Liberman, 2000) It is noted that the reaction occurs inside a thin active reaction zone, where the temperature is close to that of the burnt matter $T_{b}$. The mechanism of flame propagation may be explained as follows. Thermal conduction transports the thermal energy from the hot active reaction zone to the cooler layers of the fuel mixture, thereby heating the latter and therefore increasing the reaction rate inside it. On the other hand, the reaction rate goes down with exhaustion of the unburnt fuel. As a result, the flame front moves continuously from the burnt gas to the fresh premixture. In the present study, both fresh and burnt matters are assumed to be ideal gases.

The main flame parameters are the expansion factor $\Theta$ defined as a fresh to burnt gas density ratio $\Theta=\rho_{f} / \rho_{b}$, the unstretched laminar (planar) flame speed $U_{f}$, illustrated in Fig. 1.1, and the flame front thickness $L_{f}$ defined conventionally as

$$
L_{f}=\kappa / C_{p} \rho_{f} U_{f}
$$

where $\kappa$ is the thermal conduction coefficient and $C_{p}$ is the specific heat at constant pressure. The characteristic value of the flame front thickness is $L_{f}=\left(10^{-4} \sim 10^{-3}\right) \mathrm{cm}$, which is much smaller than the typical size of a combustion chamber $R=(10 \sim 100) \mathrm{cm}$. As a result, a flame is usually treated as a discontinuity surface separating the fresh and the burnt gases. Planar flame speed $U_{f}=\left(10 \sim 10^{3}\right) \mathrm{cm} / \mathrm{s}$, is usually much smaller than the speed of sound, such that slow combustion happens almost isobarically, $P \approx$ const . 
If one studies the macro-scale (hydrodynamic) aspects of flame propagation, then the microscale details of the chemical reaction are of minor importance, and it is often convenient to replace the detailed kinetics of all chemical processes with a single irreversible (Arrhenius) reaction. The simplest solution to the combustion equations (the Navier-Stokes and the heat conduction equations) corresponding to the planar flame front has been presented in the classical work by Zeldovich and Frank-Kamenetsky (Bychkov and Liberman, 2000). This solution determined the planar flame speed $U_{f}$ as a function of the thermal and chemical properties of the fuel mixture. Figure 1.1c shows the temperature distribution, the local mass fraction of the fresh gas $Y$, and the reaction rate $A$ scaled by its maximal value inside the burning zone. It can be seen from Fig. 1.1c that the value $L_{f}$ defined by Eq. (1.1) is just a parameter of length dimension in the problem, while the characteristic flame width may be an order of magnitude larger.

However, a planar flame illustrated by Fig. 1.1 happens very seldom in reality. Almost all industrial flames have a corrugated front shape. A corrugated front has a larger surface area; it consumes more fuel mixture per unit of time and propagates faster than a planar front would spread in the same mixture and the same thermodynamic conditions. Calculation of the curved flame velocity $U_{w}$ (called also the total burning rate) is probably the most important problem in combustion science; often, $U_{w}$ exceeds the planar flame speed $U_{f}$ by orders of magnitude. A flame front usually gets corrugated due to the intrinsic flame instabilities, external turbulent flow, combustion-acoustic coupling, flame interaction with combustor walls and many other factors. In the standard approach of an infinitely thin flame front (Landau limit), the turbulent flame velocity is proportional to the flame surface area 


$$
U_{w} / U_{f}=S_{w} / S_{f},
$$

where $S_{w}$ is the surface area of a curved flame and $S_{f}$, for instance, is the cross-section of the tube (i.e. the area a planar front would have).

\subsection{Overview \& Objectives}

\subsubsection{Overview}

During the process of deflagration-to-detonation transition (DDT), a slow subsonic flame accelerates spontaneously, with the velocity increase by 3-4 orders of magnitude, which eventually triggers explosion ahead of the flame front, and goes over into a self-sustaining detonation. This phenomenon is crucial in terrestrial conditions, in particular, in safety issues in mines, subways and power plants as well as in design of pulse-detonation engines (Roy et al., 2004). Moreover, it is also relevant to extraterrestrial, unbounded systems such as explosion fronts in supernovae (Akkerman et al., 2011; Bychkov and Liberman, 2000). Still, in spite of its extreme fundamental and technological importance, until recently DDT remained one of the most intriguing and least understood processes in combustion.

The first qualitative explanation of the flame acceleration and DDT has been suggested by Shelkin for the geometry of combustion in smooth tubes (Shelkin 1940; Shepherd and Lee, 1992). According to Shelkin, the key elements of the process are wall friction and turbulence. Specifically, the combustible gas expands with burning, which induces a flow in the fuel mixture. Being highly non-uniform due to wall friction, the induced flow bends the flame, hence increasing the fuel consumption rate and driving the flame acceleration. Additional flame distortion is provided by turbulence, which also compensates for the thermal loss to the wall. Since turbulence (including turbulent burning) belongs to the most difficult problems of modern 
science, there was almost no progress in the quantitative theoretical understanding of the flame acceleration for more than 70 years.

During the last decade it was shown that at certain conditions extremely strong flame acceleration with DDT is possible even within the regime of laminar flows, while turbulence plays a supplementary role. Various stages of flame acceleration in tubes, starting with a fingerlike flame front, and ending with fast Chapman-Jouguet deflagration and detonation triggering have been investigated (Bychkov et al., 2007; Bychkov et al., 2005; Akkerman et al., 2006; Akkerman et al., 2010; Valiev et al., 2008; Valiev et al., 2009; Bychkov et al., 2010; Bychkov et al., 2008; Valiev et al., 2010). Both 2D channels and cylindrical tubes, smooth and obstructed, were considered. The detailed study has demonstrated three different mechanisms of flame acceleration such:

1) At the early stages of flame burning at the closed tube end, the flame front acquires a finger-shape and demonstrates strong acceleration during a short time interval (Bychkov et al., 2007; Valiev et al., 2013). As illustrated in Fig. 1.2, the acceleration is terminated as soon as the flame front touches the side wall of the tube. While, for relatively slow hydrocarbon flames, this preliminary finger-flame acceleration ends with formation of the well-known "tulip flame" structure with little relation to DDT; for fast (e.g, hydrogenoxygen) flames even short-term finger-flame acceleration increase the flame propagation speed up to sonic values with important influence on the subsequent DDT process. 

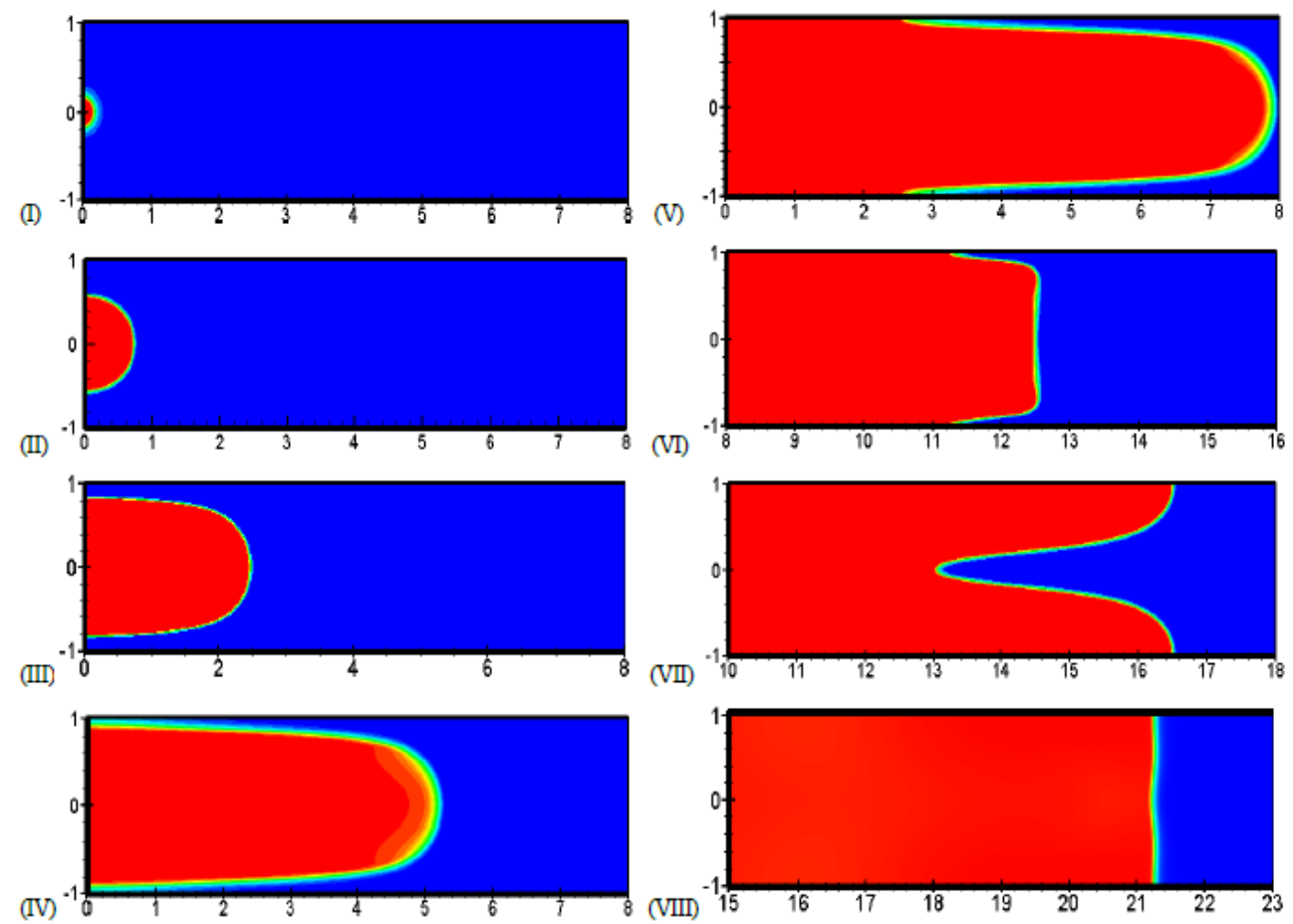

FIG. 1.2. Evolution of a finger flame front (Bychkov et al., 2007)
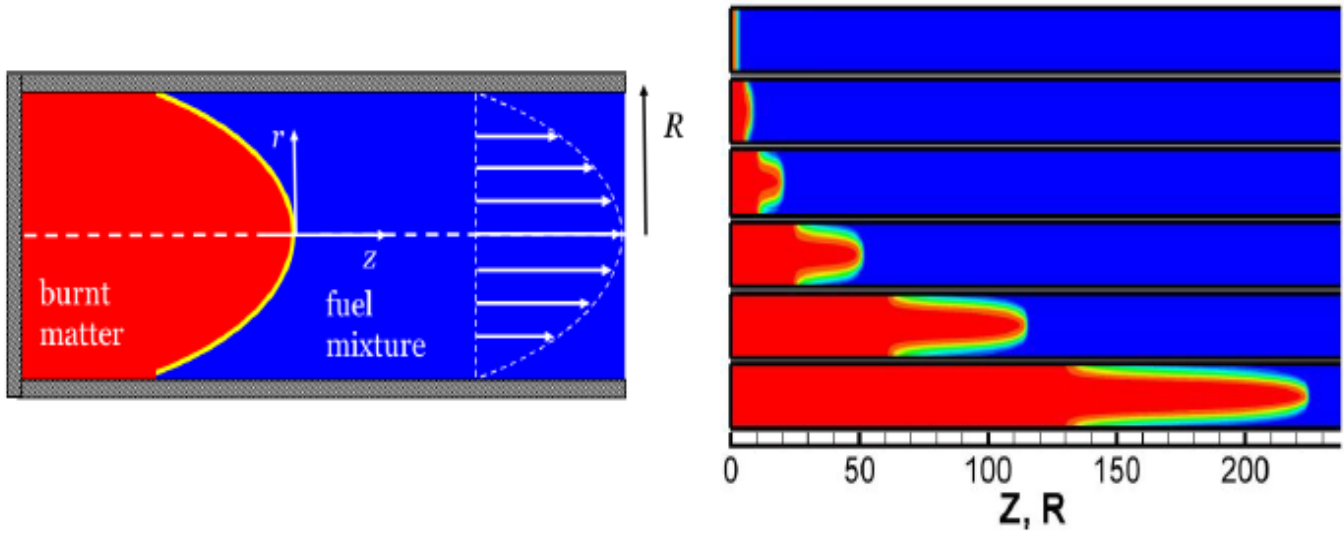

FIG. 1.3. Wall friction (Shelkin) scenario of flame acceleration in smooth tubes (Bychkov et al., 2005)

2) The classical Shelkin mechanism due to wall friction in smooth tubes (described qualitatively above and illustrated in Figs. 1.3) depends noticeably on the tube width, with the associated acceleration rate decreasing strongly with the Reynolds number. The quantitative analytical theory of the process has been developed under the set of model 
assumptions such as (i) near-isobaric, infinitely thin flame front; (ii) plane-parallel flame generated flow, (iii) exponential state of the flame acceleration. The theory was validated by extensive numerical simulations (Bychkov et al., 2005; Akkerman et al., 2006; Akkerman et al., 2010). These theoretical achievements have also been supported by refined experiments of the new type on DDT in microtubes (Wu et al., 2007; Wu and Wang 2011). The success of the theory, being in agreement with the modeling and experiments, has opened new technological possibilities of DDT in micro-combustion.

3) It is also mentioned in the past that a qualitatively new physical mechanism of extremely fast flame acceleration in channels and tubes with obstacles was recently revealed by Bychkov et al. (2008). The new mechanism is based on delayed burning between the obstacles, creating a powerful jet-flow and thereby driving the acceleration as illustrated in Figs. 1.4. This acceleration is extremely strong and independent of the Reynolds number, so the effect can be effectively utilized at industrial scales. Understanding of this mechanism provides the guide for optimization of the obstacle shape.
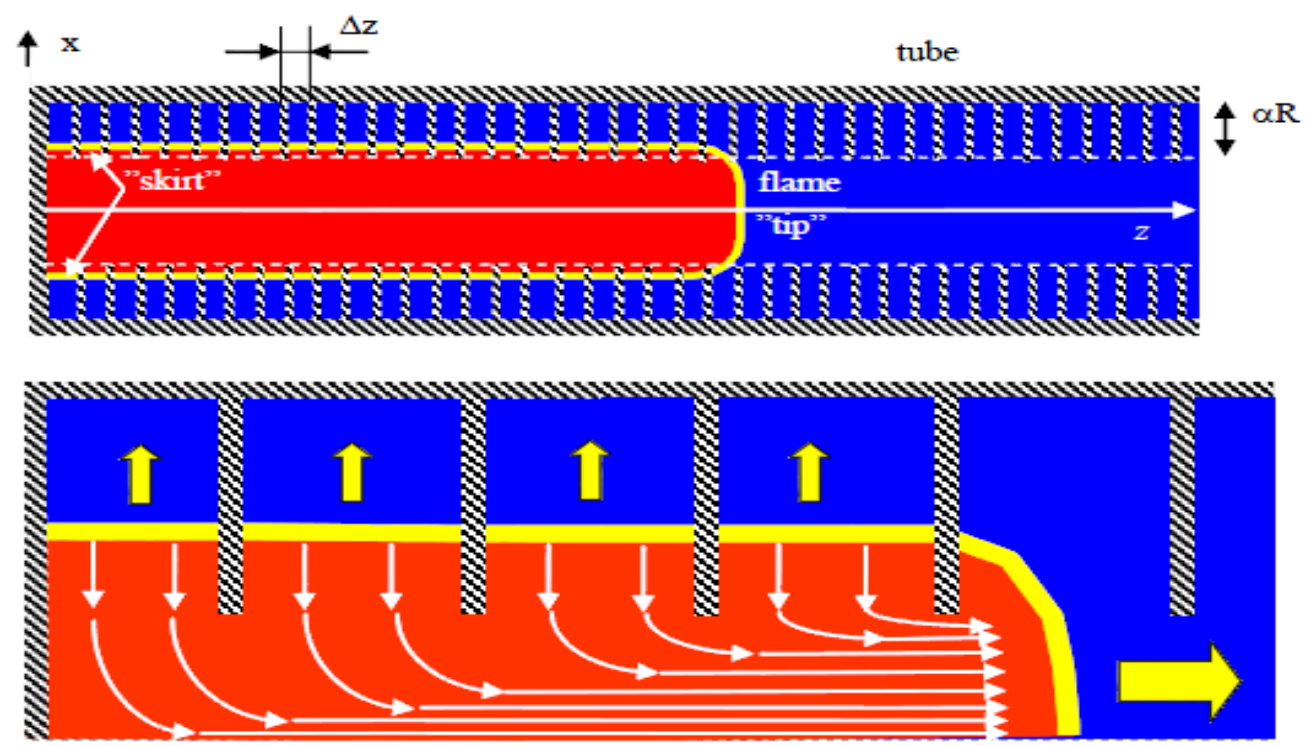

FIG. 1.4. Scheme of the physical mechanism of flame acceleration in obstructed tubes (Bychkov et al., 2008) 


\subsubsection{Objectives}

The general goal of this thesis is to shed the light on a set of phenomena within the deflagration to detonation transition (DDT) scenario. Analytical formulations (Bychkov et al., 2005; Akkerman et al., 2006) describe the acceleration of a premixed flame propagating in a horizontal micro channel/tube from its closed end to open one. Whenever, a new theory is proposed, it is important to know any intrinsic limitations given by associated approximations, and it is critical to scrutinize the accuracy of the developed theory. In this thesis, these limitations will be identified and the original formulations will be revised. On the way to this goal, the following objectives will be met:

- Determine the intrinsic limitations and accuracy of the developed theories of Bychkov and Akkerman

- Investigate the effect of thermal expansion on flame shape and acceleration utilizing direct numerical simulations (DNS) and compare the simulation results with analytical formulations

- By means of DNS, bridge the gap between the theoretical and experimental studies on ethylene/oxygen combustion (Wu and Wang, 2011) 


\section{Chapter 2: Theory of Flame Acceleration in Tubes due to Wall}

\section{Friction: Intrinsic Limitations and Accuracy}

This Chapter is devoted to the model investigation of the near-isobaric flame acceleration in tubes and channels. Specifically, in Section 2.1, the Bychkov-Akkerman formulation (Bychkov et al., 2005; Akkerman et al., 2006) is briefly summarized; the intrinsic limitations of this theory in a 2D geometry are determined in Sec. 2.2,; the findings are extended to the configuration of a cylindrical pipe in Sec. 2.3, with a quantitative analyses presented in Sec. 2.4. It is emphasized that the approach of an incompressible flow is adopted in Chapter 2; the effect of gas compression on the near-sonic flame propagation will be discussed later in Chapter 5.

\subsection{Basics of the Bychkov-Akkerman formulation}

First of all, the basics of the incompressible analytical theories (Bychkov et al., 2005; Akkerman et al., 2006) devoted to the 2D and cylindrically-axisymmetric configurations, respectively. The original formulation is based on the following major approximations:

(i) Near-isobaric combustion process;

(ii) Zero flame thickness;

(iii) Plane-parallel flame-generated flow in the unburned gas, $\mathbf{u}=\mathbf{e}_{z} u_{z}(r, t)$, with the burnt gas being at rest; and

(iv) Exponential state of the flame acceleration.

Specifically, an infinitely thin premixed laminar flame front that spreads locally with the normal velocity $U_{f}$ with respect to the fuel mixture is considered. Such a flame propagates in a 2D channel of half-width $R$ or a cylindrical tube of radius $R$, with adiabatic $(\mathbf{n} \cdot \nabla T=0)$ non-slip 
( $\mathbf{u}=0$ ) walls, where $\mathbf{n}$ is a normal vector at the wall. One end of the tube/channel is closed, and the flame propagates from the closed end to the open one, as illustrated in Fig. 2.1.

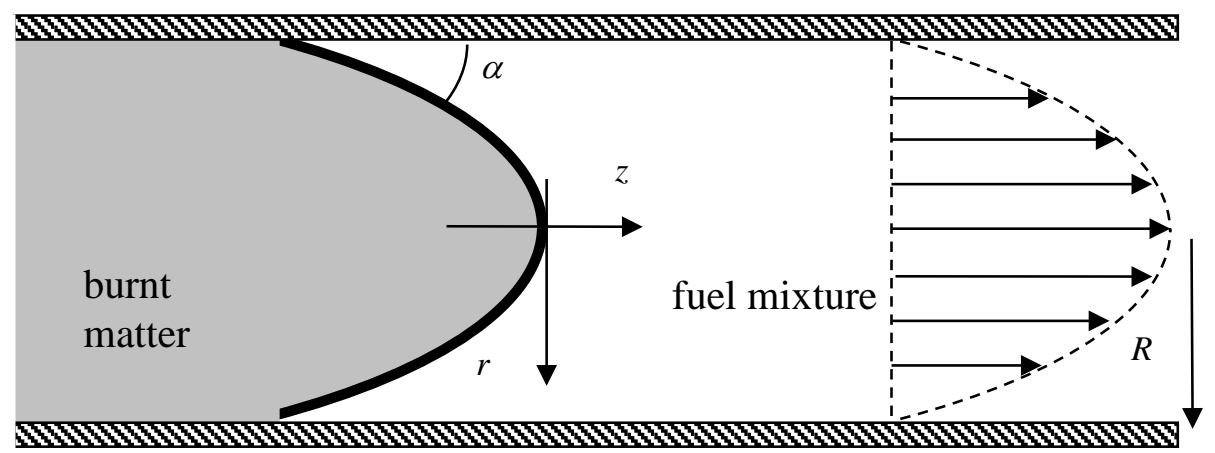

FIG. 2.1. A flame in a tube or channel with non-slip at the walls

To simplify the calculations, the standard dimensionless variables $(\eta ; \xi)=(x ; z) / R, \tau=t U_{f} / R$, $\mathbf{w}=\mathbf{u} / U_{f}$ are introduced. The density and pressure are scaled by the fuel quantities $\rho_{f}$ and $\rho_{f} U_{f}^{2}$, respectively. The temperature is scaled by that associated with the fuel, $\vartheta=T / T_{f}$, $1 \leq \vartheta \leq \Theta$, with $\vartheta=1$ and $\vartheta=\Theta$ in the fresh and burnt gases, respectively. The average molar weights of the unburned and burnt gases are assumed to be equal such that the expansion factor $\Theta$ is also associated with the fresh to burnt gas density ratio $\Theta=\rho_{f} / \rho_{b}$. Viscosity effects are characterized by the Reynolds number related to the flame propagation, $\operatorname{Re}=U_{f} R / v$, where $v$ is the kinematical viscosity.

A traditional evaluation that the total burning rate is simply proportional to the total surface area of the flame front is employed. In a $2 \mathrm{D}$ geometry it reads $U_{w} / U_{f}=S_{2 D} / 2 R$, and a $2 \mathrm{D}$ "volume” of the burning gas increases by $(\Theta-1) 2 R U_{w}$ per unit time. In a cylindrical geometry 
it is calculated as $U_{w} / U_{f}=S_{c y l} / 2 \pi R^{2}$, with a volumetric increment of the burnt gas per unit time being $(\Theta-1) 2 \pi R^{2} U_{w}$. In both configurations, the average combustion-generated flow velocity is related to the total burning rate as

$$
\left\langle u_{z}\right\rangle=(\Theta-1) U_{w}, \quad \text { or } \quad\left\langle w_{z}\right\rangle=(\Theta-1) \Omega_{w},
$$

where the scaled total burning rate $\Omega_{w}=U_{w} / U_{f}$ is adopted.

Obviously, the flow of the fuel mixture in a tube/channel is non-uniform. Indeed, the gas stops at the non-slip walls because of friction and reaches the maximal velocity at the tube axis. Such a flow distorts the flame shape, increases the flame surface area and propagation speed. According to Eq. (2.1), this renders an additional increase of the flow velocity. Consequently, one arrives at a positive flow-flame feedback: the faster the flow, the stronger the distortion of the flame shape, and the larger the flame velocity. Therefore, the flame accelerates. Asymptotically, it accelerates exponentially in time

$$
U_{w} \propto \exp \left(\sigma U_{f} t / R\right), \quad \Omega_{w} \propto \exp (\sigma \tau),
$$

where the dimensionless growth rate $\sigma$ is an eigenvalue that will be determined below.

The model of a plane-parallel, flame-generated flow is self-consistent if the pressure gradient is a function of time only, $\partial p / \partial \xi=\Pi(\tau)$. Then the plane-parallel Navier-Stokes equation reads

$$
\frac{\partial w_{z}}{\partial \tau}=\Pi(\tau)+\frac{1}{\operatorname{Re}} \frac{\partial^{2} w_{z}}{\partial \eta^{2}} \quad \text { (a), } \quad \text { or } \quad \frac{\partial w_{z}}{\partial \tau}=\Pi(\tau)+\frac{1}{\operatorname{Re}} \frac{1}{\eta} \frac{\partial}{\partial \eta}\left(\eta \frac{\partial w_{z}}{\partial \eta}\right) \quad \text { (b). }
$$

in a 2D and cylindrical-axisymmetric configurations, respectively, with $\Pi(\tau) \propto \Omega_{w} \propto \exp (\sigma \tau)$. The flame surface is described by a dimensionless function $\xi(\eta, \tau)=g(\tau)+f(\eta, \tau)$, where $g$ quantifies the evolution of the flame tip and $f$ describes the flame shape with respect to it, i.e. 
the deviation of the flame shape from the planar one. Consequently, every local segment of the flame front propagates with the velocity $w_{f}(\eta, \tau)=\sqrt{1+(\partial f / \partial \eta)^{2}}$ in the $\xi$-direction with respect to the fuel mixture, with $w_{f}(0, \tau)=1$ and $\left\langle w_{f}\right\rangle=\Omega_{w}$. In addition, both the flame and the fuel are drifted by the flame-generated flow, Eq. (2.1). As a result, the flame evolution equation reads

$$
w_{z}(0, \tau)-w_{z}(\eta, \tau)=\sqrt{1+\left(\frac{\partial f}{\partial \eta}\right)^{2}}-1-\frac{\partial f}{\partial \tau} \approx \frac{\partial f}{\partial \eta}-\frac{\partial f}{\partial \tau} .
$$

Equations (2.1) - (2.4) state the backbone of the formulations (Bychkov et al., 2005; Akkerman et al., 2006; Akkerman et al., 2010).

\subsection{Flame acceleration in a $2 \mathrm{D}$ channel}

Here it is focused on a 2D geometry. The system (2.1), (2.2), (2.3a) has the solution

$$
\begin{aligned}
& w_{z}=(\Theta-1) \Omega_{w} \frac{\cosh \mu-\cosh (\mu \eta)}{\cosh \mu-\mu^{-1} \sinh \mu}, \\
& \left\langle w_{z}\right\rangle=(\Theta-1) \Omega_{w} \propto \frac{\cosh \mu-\mu^{-1} \sinh \mu}{\cosh \mu-1} \exp (\sigma \tau),
\end{aligned}
$$

where $\mu=\sqrt{\sigma \operatorname{Re}}$. The flame shape is searched in the form $f(\eta, \tau)=\Phi(\eta) \exp (\sigma \tau)$, with $\Phi(0)=0$. Then Eqs. (2.4), (2.5) and (2.6) yield (Bychkov et al., 2005)

$$
\Phi=\frac{(\Theta-1) \Phi(1)}{\cosh \mu-\mu^{-1} \sinh \mu}\left(\frac{\exp (\mu \eta)}{2(\mu+\sigma)}-\frac{\exp (-\mu \eta)}{2(\mu-\sigma)}+\frac{\mu^{2}}{\mu^{2}-\sigma^{2}} \frac{\exp (-\sigma \eta)}{\sigma}-\frac{1}{\sigma}\right)
$$

With the boundary condition $\Phi(\eta)=\Phi(1)$ at $\eta=1$, Eq. (2.7) acquires the form

$$
\frac{\mu \cosh \mu-\sinh \mu}{\mu(\Theta-1)}=\frac{\exp \mu}{2(\mu+\sigma)}-\frac{\exp (-\mu)}{2(\mu-\sigma)}+\frac{\mu^{2}}{\mu^{2}-\sigma^{2}} \frac{\exp (-\sigma)}{\sigma}-\frac{1}{\sigma} .
$$


Equation (2.8) is the major result of the incompressible 2D formulation (Bychkov et al., 2005): it couples the flame acceleration rate $\sigma$ to the thermal expansion factor $\Theta=\rho_{f} / \rho_{b}$ and the flame propagation Reynolds number $\operatorname{Re}=R U_{f} / v$, through the intermediate parameter $\mu=\sqrt{\sigma \mathrm{Re}}$. The study (Bychkov et al., 2005) moved further: in the limit of large $\Theta$, the leading-order reduction of Eq. (2.8) can be solved analytically. Indeed, as verified below, a large $\Theta$ corresponds to a large $\mu$, and for $\mu>>1$ Eq. (2.8) reduces to

$$
\mu+\sigma=(\Theta-1) \frac{\mu}{\mu-1}
$$

with the solution

$$
\sigma=\frac{(\operatorname{Re}-1)^{2}}{4 \operatorname{Re}}\left(\sqrt{1+\frac{4 \operatorname{Re} \Theta}{(\operatorname{Re}-1)^{2}}}-1\right)^{2}, \quad \mu=\sqrt{\sigma \operatorname{Re}}=\frac{\operatorname{Re}-1}{2}\left(\sqrt{1+\frac{4 \operatorname{Re} \Theta}{(\operatorname{Re}-1)^{2}}}-1\right)
$$

For large Reynolds numbers, $\operatorname{Re}>>4 \Theta$, the result (2.10) further degenerates to $\sigma=\Theta^{2} / \operatorname{Re}$, $\mu=\Theta$. It is seen now that the approach of large $\mu$ and Re intrinsically requires $\Theta$ to be large as well.

It is emphasized that Eq. (2.10) is based on the assumption of $\mu \gg>1$. What does this actually mean, and what physical parameters would obey this criterion? Of course, one can formally verify the validity of the theory by computing $\sigma$ and $\mu$, Eq. (2.10), for any given $\Theta$ and Re, and subsequently conclude if such a value of $\mu$ is really large or not. However, the very concept of $\mu \gg>1$ needs some quantification as well. Indeed, which $\mu$ is "large enough": $5,10,50,250$, 1000 or larger? Moreover, how does the violation of the limit of large $\mu$ influence the accuracy of the model? Does this mean that the case of $\mu \sim 1$ or, say, $\mu \sim 5$ surely breaks the entire approach (Bychkov et al., 2005) or not necessarily? Besides, it is realized that the dependences 
of $\mu$ versus $\Theta$ and Re can demonstrate different trends in terms of the accuracy limitations. So far, the asymptotical result (2.10) used to be validated by direct numerical simulations (Bychkov et al., 2005). While the results of these simulations agreed with Eq. (2.10) very well, such agreement was quite expected since the simulations were performed in the domain of large Re and $\Theta$, yielding $\mu>>1$. Would it be the case otherwise?

Furthermore, a comparison to a "computational experiment" cannot be treated as a rigorous mathematical validation of an analytical theory. As a result, the primary task of this part of the thesis is to determine the intrinsic limitations and accuracy of the theory above, in general, and Eq. (2.10), in particular. For this purpose, Eq. (2.8) has been solved computationally, and the detailed comparison of the results obtained to the prediction of formula (2.10) has been done. A MATLAB ${ }^{\circledR}$ program was written to solve Eq. (2.8) for the flame acceleration rate numerically. The program utilizes the Newton-Raphson method - a standard powerful method to solve equations numerically, which are hard to be solved analytically. (See appendix A for details)

It is recalled in this respect that Eq. (2.8) is also based on a set of model assumptions; see Sec. 2.1. Nevertheless, it is a much less speculative equation as compared to Eq. (2.10) since Eq. (2.8) does not require any limitations on the physical quantities Re and $\Theta$.

This numerical solution to Eq. (2.8) is compared to the analytical prediction of (2.10) in Fig. 2.2. Specifically, Fig. 2.2 shows the acceleration rate $\sigma$ versus the flame propagation Reynolds number Re at the fixed thermal expansion coefficients $\Theta=4 ; 6 ; 8 ; 10$. Equations (2.8) and (2.10) are presented by the dashed and solid lines, respectively. 


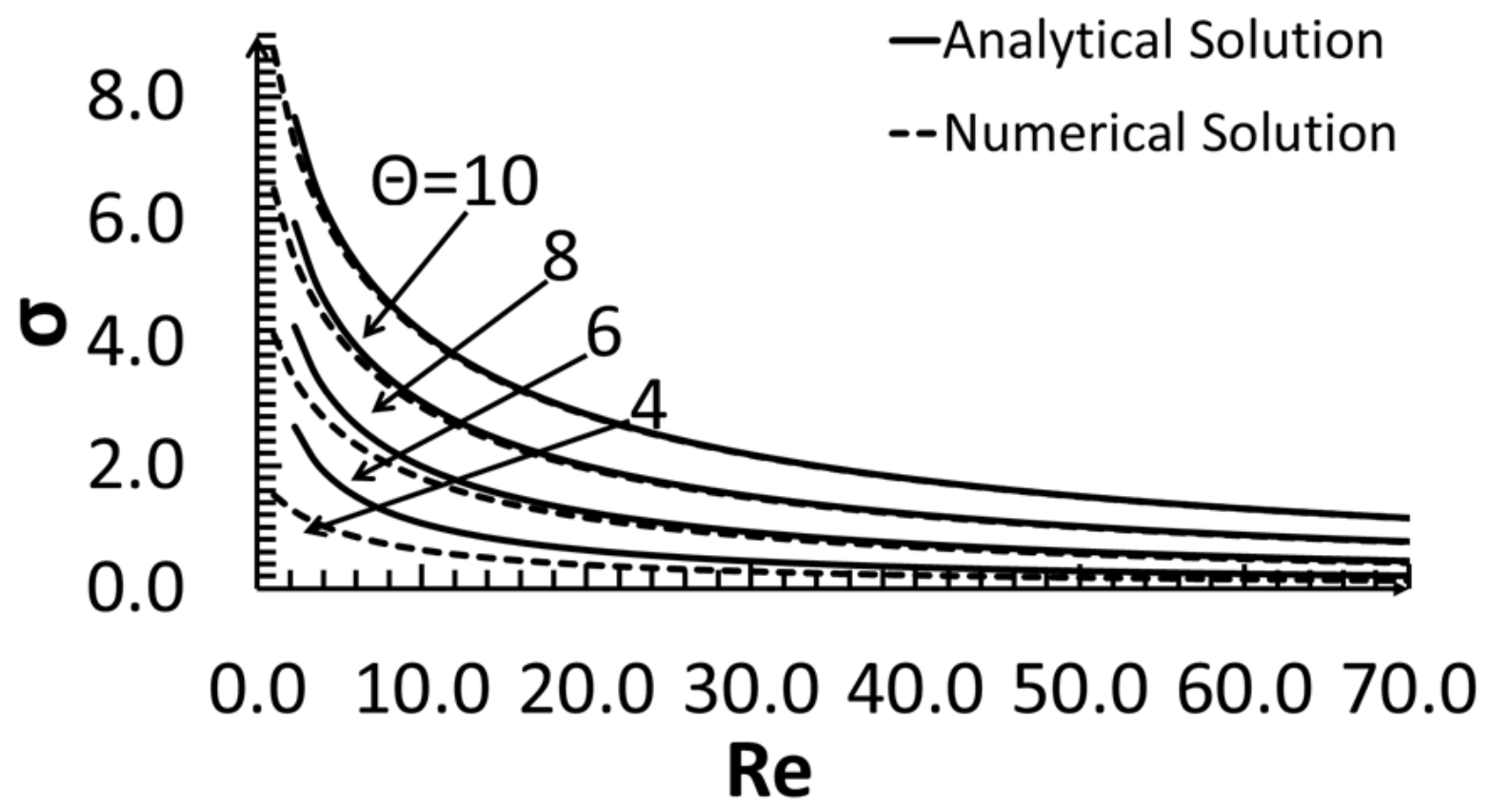

FIG. 2.2. The flame acceleration rate $\sigma$ versus the flame propagation Reynolds number Re at the fixed thermal expansion coefficients $\Theta=4 ; 6 ; 8 ; 10$. Equations (2.8) and (2.10) are shown by dashed and solid lines, respectively

It is seen that $\sigma$ grows with the decrease in Re, and one can observe very good agreement between the analytical approximation and the numerical results when thermal expansion large enough, $\Theta=8 \sim 10$. However, this agreement deteriorates as soon as $\Theta$ decreases. In particular, the discrepancy between these two solution is significant when both $\Theta$ and Re are small, say, $\Theta=4$ and $\operatorname{Re}<10$. The same effect is observed in Fig. 2.3, where $\sigma$ is plotted versus $\Theta$ at fixed $\operatorname{Re}=5 ; 10 ; 20$. 


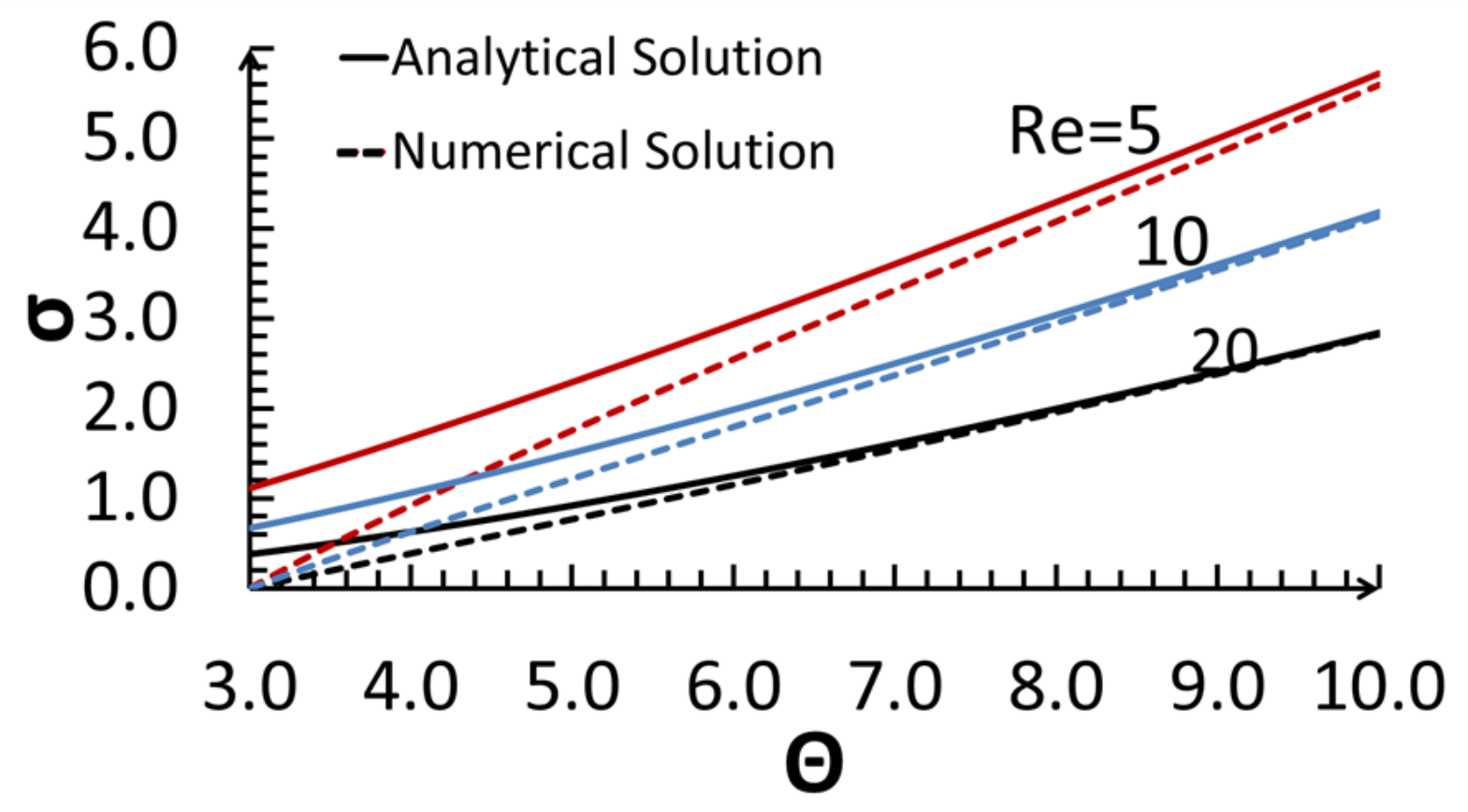

FIG. 2.3. The flame acceleration rate $\sigma$ versus the thermal expansion coefficient $\Theta$ at the fixed flame propagation Reynolds numbers $\mathrm{Re}=5 ; 10 ; 20$. Equations (2.8) and (2.10) are shown by dashed and solid lines, respectively

It is seen that $\sigma$ grows with $\Theta$, and the results of (2.8) and (2.10) coincide at large expansion factors and Reynolds numbers. However, they considerably differ at small $\Theta$. Indeed, while Eq. (2.10) shows a near-parabolic dependence of $\sigma$ versus $\Theta$, the numerical solution to Eq. (2.8) yields $\sigma \rightarrow 0$ when $\Theta \rightarrow 3$. The latter trend also supports the formulation on steady flame propagation (Akkerman et al., 2010) yielding that a flame surely accelerate in a 2D channel with one end closed if $\Theta>3$. Moreover, this discussion supports the recent study on the possibility of near-sonic, quasi- steady flame propagation (Valiev et al., 2013). In this respect, Fig. 2.3 qualitatively resembles Figure 2 of (Valiev et al., 2013). Besides, Fig. 2.3 demonstrates that the discrepancy between the analytical approximation and the numerical solution gets stronger with 
the decrease in the flame propagation Reynolds number, and thereby one cannot expect a good predictability from the theory (Bychkov et al., 2005) at small Re.

A detailed analysis of such a discrepancy (the "error") between the numerical solution to Eq. (2.8) and its analytical approximation (2.10) has been performed, with a relative error between the numerical and analytical results given by

$$
\operatorname{Error}(\%)=\frac{\left|\sigma_{\text {numerical }}-\sigma_{\text {analytical }}\right|}{\sigma_{\text {numerical }}} * 100,
$$

where $\sigma_{\text {analytical }}$ and $\sigma_{\text {numerical }}$ in Eq. (2.11) represent the analytical, Eq. (2.10), and numerical solutions to Eq. (2.8), respectively. Figure 2.4a shows the relative error, Eq. (2.11), versus $\Theta$ at fixed $\operatorname{Re}=5 ; 10 ; 20$, whereas Fig. 2.4b presents the relative error versus $\operatorname{Re}$ at $\Theta=4 ; 6 ; 8 ; 10$. It is clearly seen that the error diminishes with the increase in $\Theta$ and/or Re.

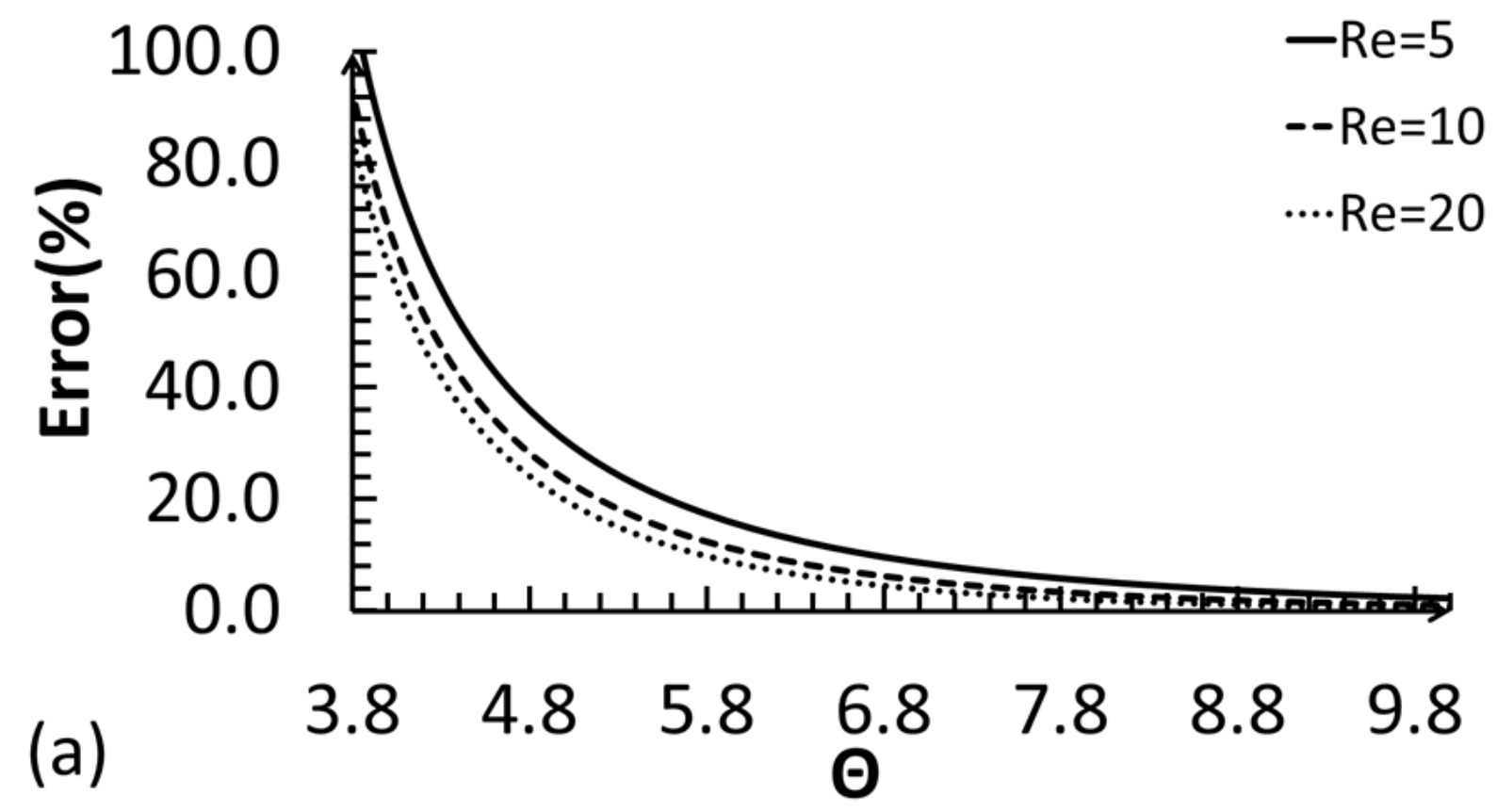




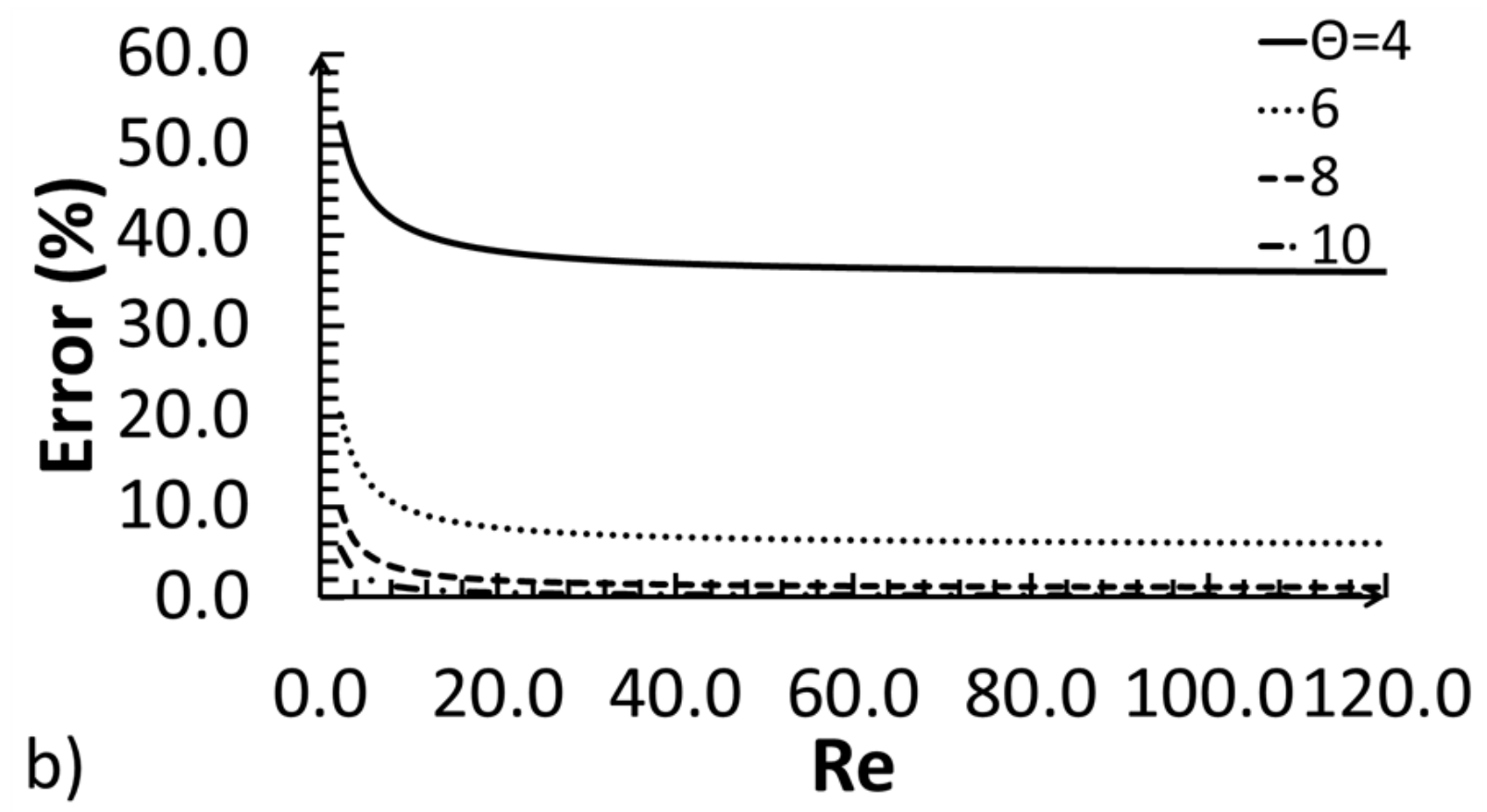

FIG. 2.4. Relative error (in \%) versus thermal expansion coefficient $\Theta$ at fixed propagation Reynolds numbers such as $\mathrm{Re}=20,10$ and 5 shown by dotted, dashed and solid lines, respectively (a); and the relative error percentage versus propagation Reynolds number at fixed thermal expansion such as $\Theta=10$, 8, 6 and 4 shown by solid \&dotted, dashed, dotted and solid lines, respectively (b)

The results shown in Fig. 2.4 lead to the intrinsic limitations of the theory, which are demonstrated by the validity domains in Fig. 2.5: the contour plot analysis of the flame propagation Reynolds number Re versus the thermal expansion $\Theta$. The error isolines (in \%) show the accuracy domains in the Re- $\Theta$ space. It is seen that the relative error is very small $(<1 \%)$ in the top right corner of this diagram, when both $\Theta$ and Re are large. On the other hand, the error grows with the decrease in Re and $\Theta$, exceeding $50 \%$ in the bottom left corner of the Re- $\Theta$. Obviously, the applicability of the theory (Bychkov et al., 2005) is doubtful in this domain. 


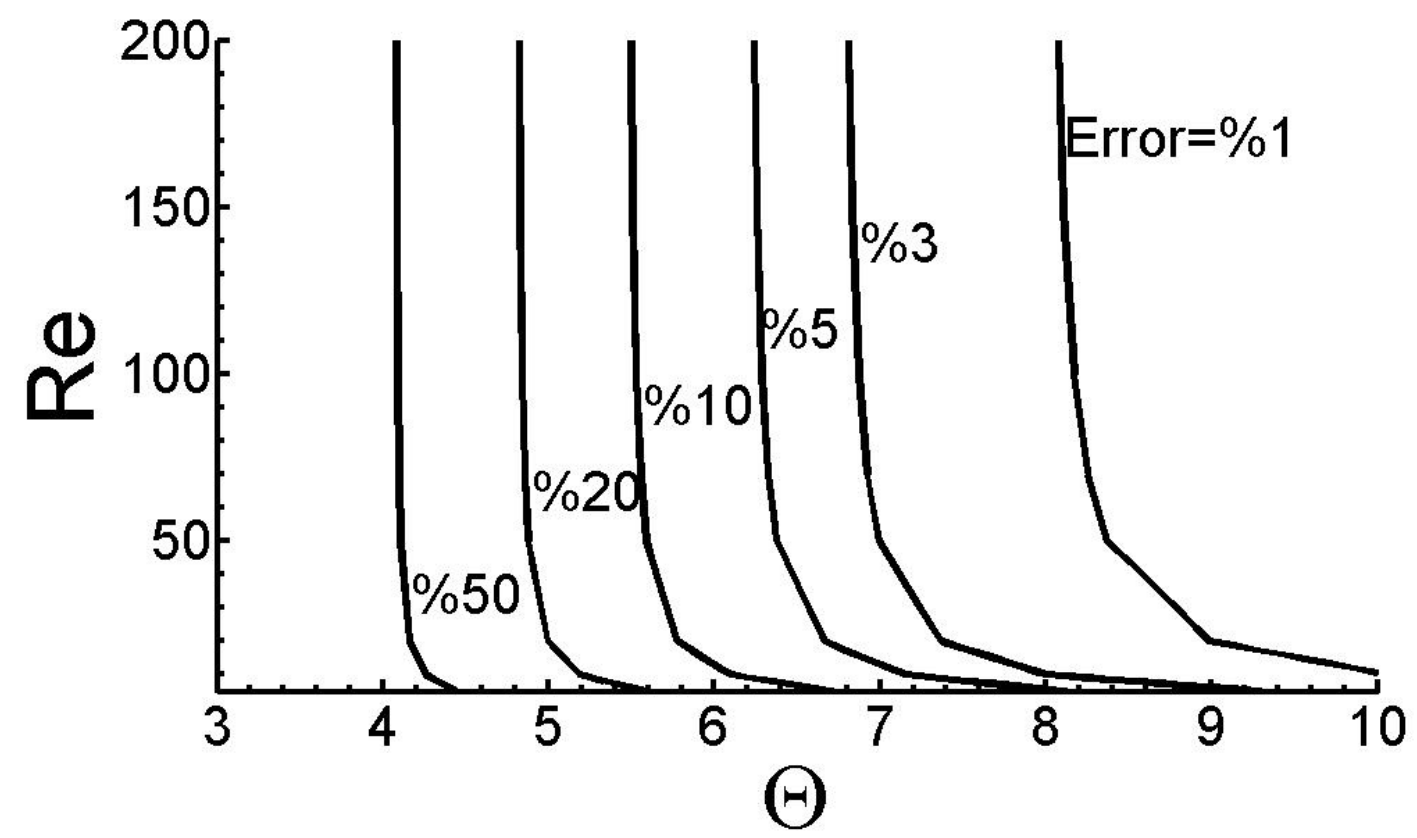

FIG. 2.5. Contour scheme (the error isolines (in \%)) demonstration of Propagation Reynolds number versus thermal expansion $\Theta$

\subsection{Flame acceleration in a cylindrical tube}

In the cylindrical axisymmetric geometry (Akkerman et al., 2006), the counterpart of Eqs. (2.5) and (2.6) reads

$$
w_{z}=(\Theta-1) \Omega_{w} \frac{I_{0}(\mu)-I_{0}(\mu \eta)}{I_{0}(\mu)-2 \mu^{-1} I_{1}(\mu)},\left\langle w_{z}\right\rangle=(\Theta-1) \Omega_{w} \propto \frac{I_{0}(\mu)-I_{0}(\mu \eta)}{I_{0}(\mu)-1} \exp (\sigma \tau),
$$

where $I_{0}(\eta)$ and $I_{1}(\mu)$ are the modified Bessel function of $0^{\text {th }}$ and $1^{\text {st }}$ orders. Similar to Sec. 2.3, the flame shape is searched in the form $f(\eta, \tau)=\Phi(\eta) \exp (\sigma \tau)$. The cylindrical-axisymmetric equation for $\Phi(\eta)$ is

$$
\sigma \Phi+\Phi^{\prime}=2(\Theta-1)\left[\Phi(1)-\int_{0}^{1} \Phi(\chi) d \chi\right] \frac{I_{0}(\mu \eta)-1}{I_{0}(\mu)-2 \mu^{-1} I_{1}(\mu)},
$$

with the solution 


$$
\Phi(\eta)=\Phi(1) \frac{[\sigma \Psi(\eta)+1] \exp (-\sigma \eta)-1}{[\sigma \Psi(1)+1] \exp (-\sigma)-1}, \quad \Psi(\eta)=\int_{0}^{\eta} I_{0}(\mu \chi) \exp (\sigma \chi) d \chi
$$

Eventually, the result (2.14) yields

$$
\frac{I_{0}(\mu)-2 \mu^{-1} I_{1}(\mu)}{2(\Theta-1)}=\frac{(\sigma+1) \exp (-\sigma)-1}{\sigma^{2}}+\Psi(1) \exp (-\sigma)-\int_{0}^{1} \Psi(\eta) \exp (-\sigma \eta) d \eta
$$

In the $0^{\text {th }}$ order approximation of $\mu^{-1}: I_{0}(\mu) \approx I_{1}(\mu) \approx \exp \mu / \sqrt{2 \pi \mu}, I_{0}(\mu \eta) \approx \exp (\mu \eta) / \sqrt{2 \pi \mu \eta}$, and Eq. (14) reduces to $\mu_{0}+\sigma_{0}=2(\Theta-1)$ (Akkerman et al., 2006), with the analytical solution

$$
\mu_{0}=\frac{\operatorname{Re}}{2}\left(\sqrt{1+\frac{8(\Theta-1)}{\operatorname{Re}}}-1\right), \quad \sigma_{0}=\frac{\mu_{0}^{2}}{\operatorname{Re}}=\frac{\operatorname{Re}}{4}\left(\sqrt{1+\frac{8(\Theta-1)}{\operatorname{Re}}}-1\right)^{2} .
$$

For $\operatorname{Re}>>8(\Theta-1)$, Eq.(2.16) further degenerates to $\mu_{0}=2(\Theta-1), \sigma_{0}=4(\Theta-1)^{2} / \operatorname{Re}<<\mu$. Equation (2.15) has been solved computationally in the same manner as Eq. (2.8). Figure 2.6a shows $\sigma$ versus Re at fixed $\Theta=4 ; 6 ; 8$. The computational solution to Eq. (2.15) and the analytical prediction (2.16) are presented by the dashed and solid lines, respectively. As expected, the acceleration intensifies with the increase in $\Theta$ and the decrease in Re; and in the cylindrical-axisymmetric configuration the acceleration rate is almost thrice higher the 2D one for the same parameters. It is shown that while solid and dashed lines qualitatively resemble each other, the quantitative agreement between them is quite poor. Moreover, unlike Sec. 2.3, the discrepancy between these two solutions gets much stronger when Re 100. Accordingly, the same effects are seen in Fig. 2.6b, where $\sigma$ is plotted versus $\Theta$ at fixed $\operatorname{Re}=5 ; 10 ; 20$. 

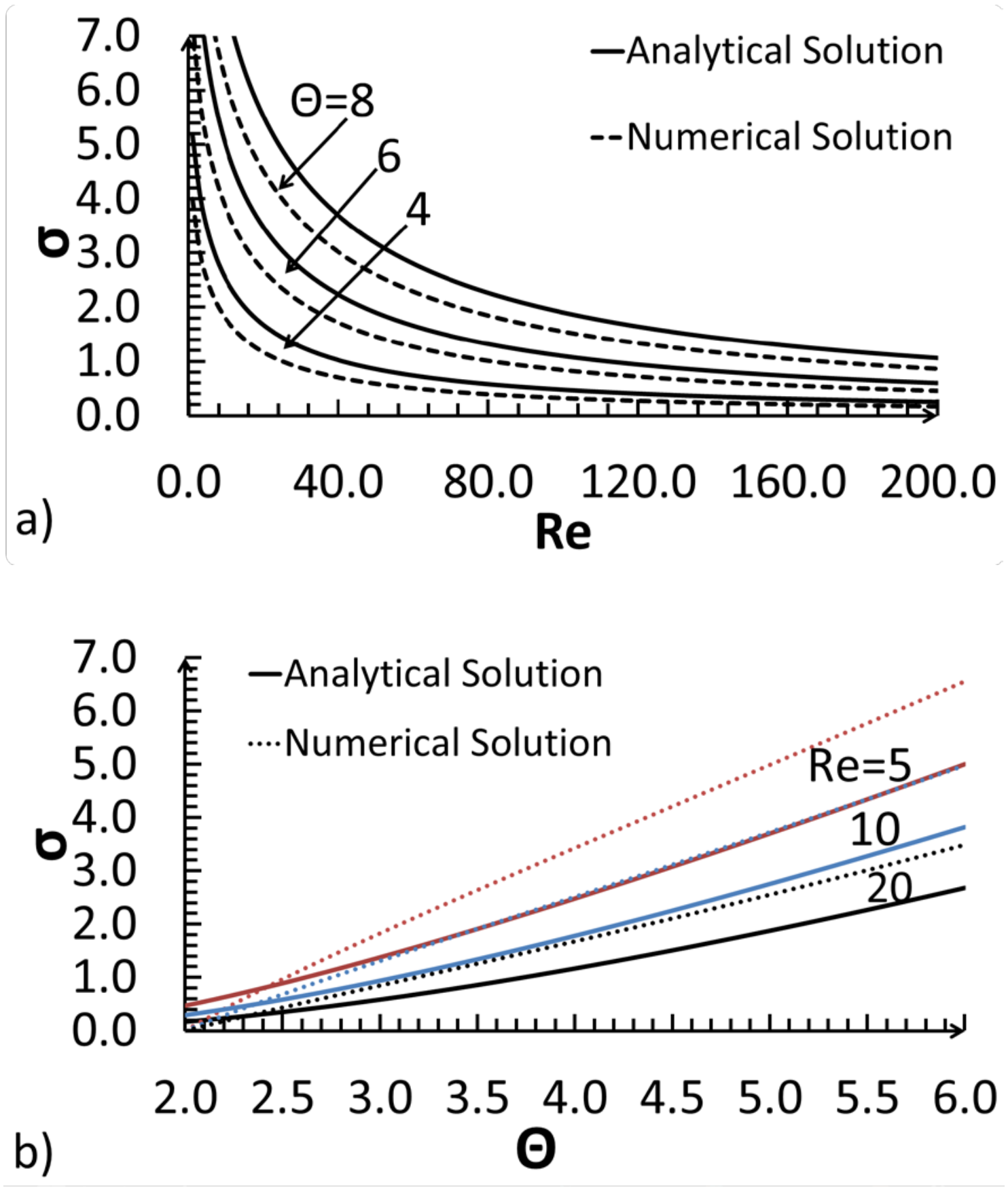

FIG. 2.6. $\sigma$ versus Re at $\Theta=4 ; 6 ; 8$ (a), and versus $\Theta$ at $\operatorname{Re}=5 ; 10 ; 20$ (b). Equations (2.15) and (2.16) are shown by dashed and solid lines, respectively

Similar to Sec. 2.3, the difference between the numerical solution and the analytical approach is quantified in Fig. 2.7. Specifically, Fig. 2.7a shows the relative error, given by Eq. (2.11), versus 
$\Theta$ at $\operatorname{Re}=5 ; 10 ; 20$, while Fig. 2.7b presents the relative error versus $\operatorname{Re}$ at $\Theta=3 ; 4 ; 6$. It is seen that agreement between the numerical solution and the $0^{\text {th }}$ order approximation is quite poor everywhere.
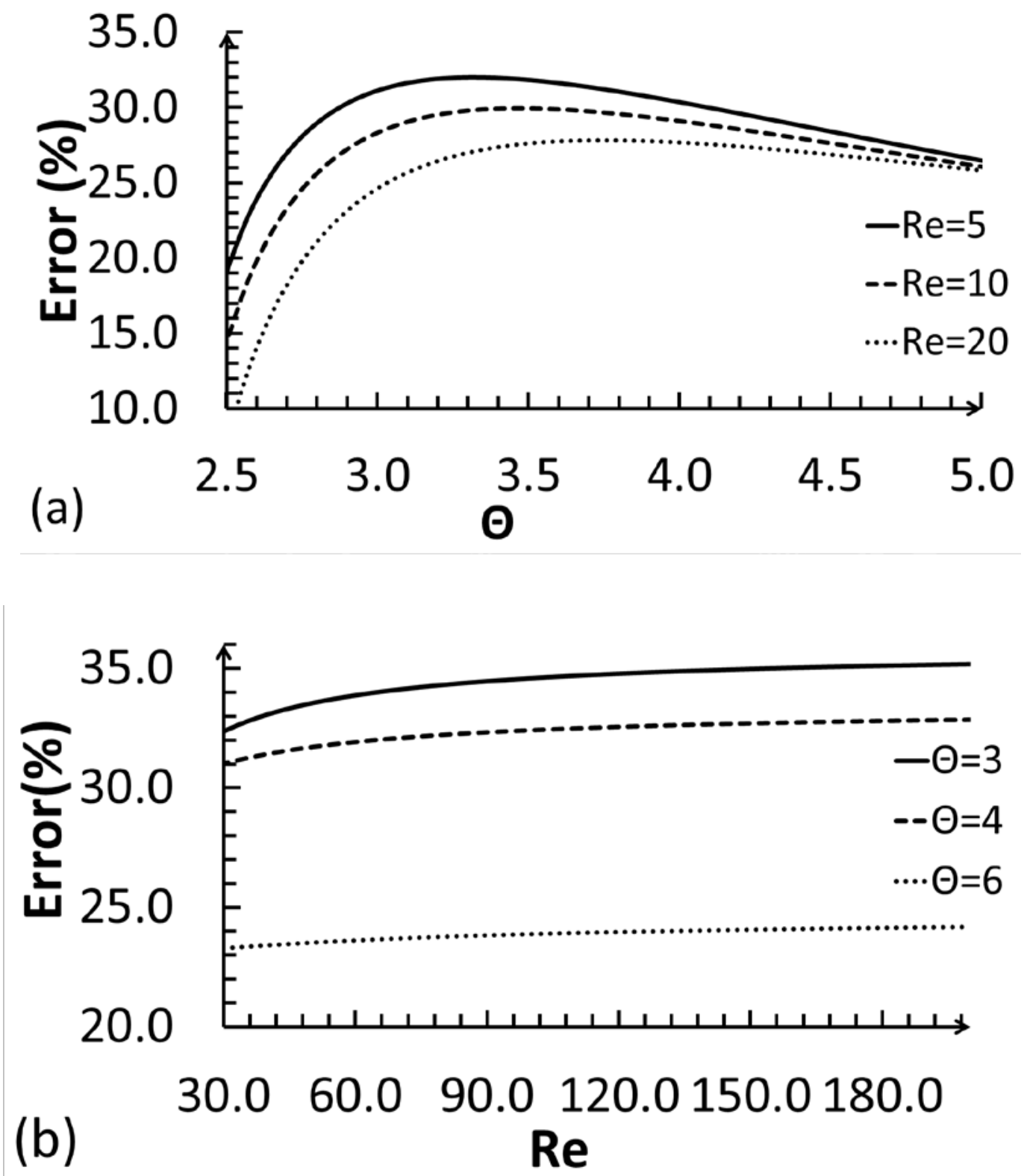

FIG. 2.7. The relative error (in \%), Eq. (2.11), versus $\Theta$ at $\operatorname{Re}=5,10$, 20, shown by solid, dashed and dotted lines, respectively (a); and versus Re at $\Theta=3,4,6$ shown by solid, dashed and dotted lines, respectively (b) 
The results shown in Fig. 2.7 provide the intrinsic limitations of Eq. (2.16) in the Re- $\Theta$ diagram, Fig. 2.8, which represents the counterpart of Fig. 2.5 for the cylindrical configuration, with the analytical prediction given by the $0^{\text {th }}$ order approximation, Eq. (2.16). The error isolines (in \%) show the accuracy domains in the Re- $\Theta$ space. It is seen that even both Re and $\Theta$ are large, the error is still very high: from $~ 24 \%$ in the bottom right corner of the diagram till $>34 \%$ in the top left corner. Consequently, the quantitative applicability of Eq. (2.16) is very questionable.

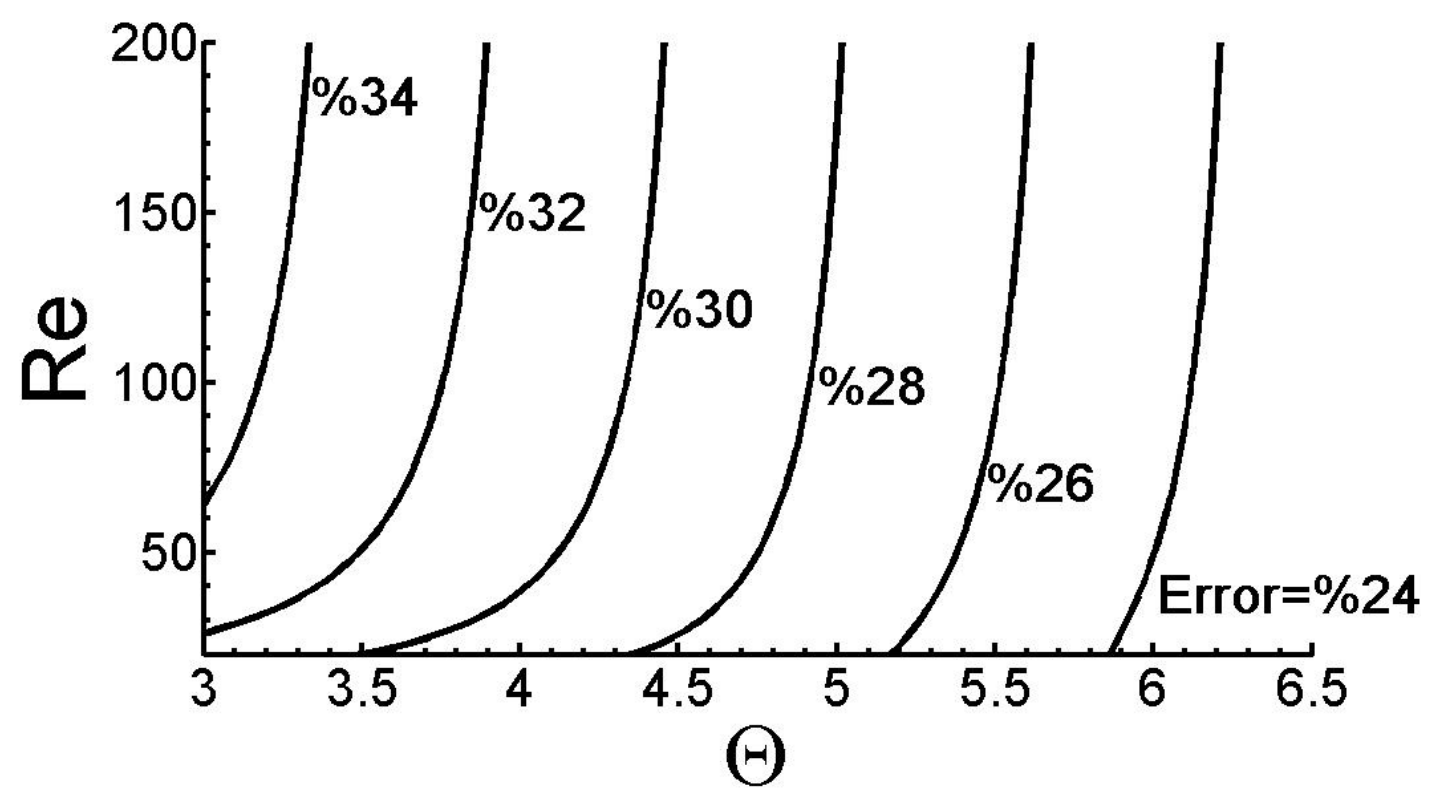

FIG. 2.8. Contour scheme (the error isolines (in \%)) demonstration in the Re- $\Theta$ diagram

Next, it is demonstrated that the analytical prediction can be improved, substantially, if the formulation is extended to the $1^{\text {st }}$ order approximation of $\mu^{-1}<<1$, namely

$$
\sigma_{1}=\frac{\operatorname{Re}}{4}\left(\sqrt{1+\frac{8(\Theta-1)}{\operatorname{Re}}\left[1+\frac{1}{\mu_{0}}+\frac{1}{2\left(\mu_{0}+\sigma_{0}\right)}\right]}-1\right)^{2} .
$$

Fig. 2.9 compares Eq. (2.17) and the numerical solution to Eq. (2.15). Specifically, Fig. 2.9a presents $\sigma$ versus $\operatorname{Re}$ at $\Theta=4 ; 6 ; 8$. Equations (2.15) and (2.17) are shown by the solid and 
dashed lines, respectively. This time one can observe much better agreement between the analytical and computational results as compared to Fig. 2.6a. The same trend is observed in Fig. 2.9b, where $\sigma$ is plotted versus $\Theta$ at $\operatorname{Re}=5 ; 10 ; 20$.
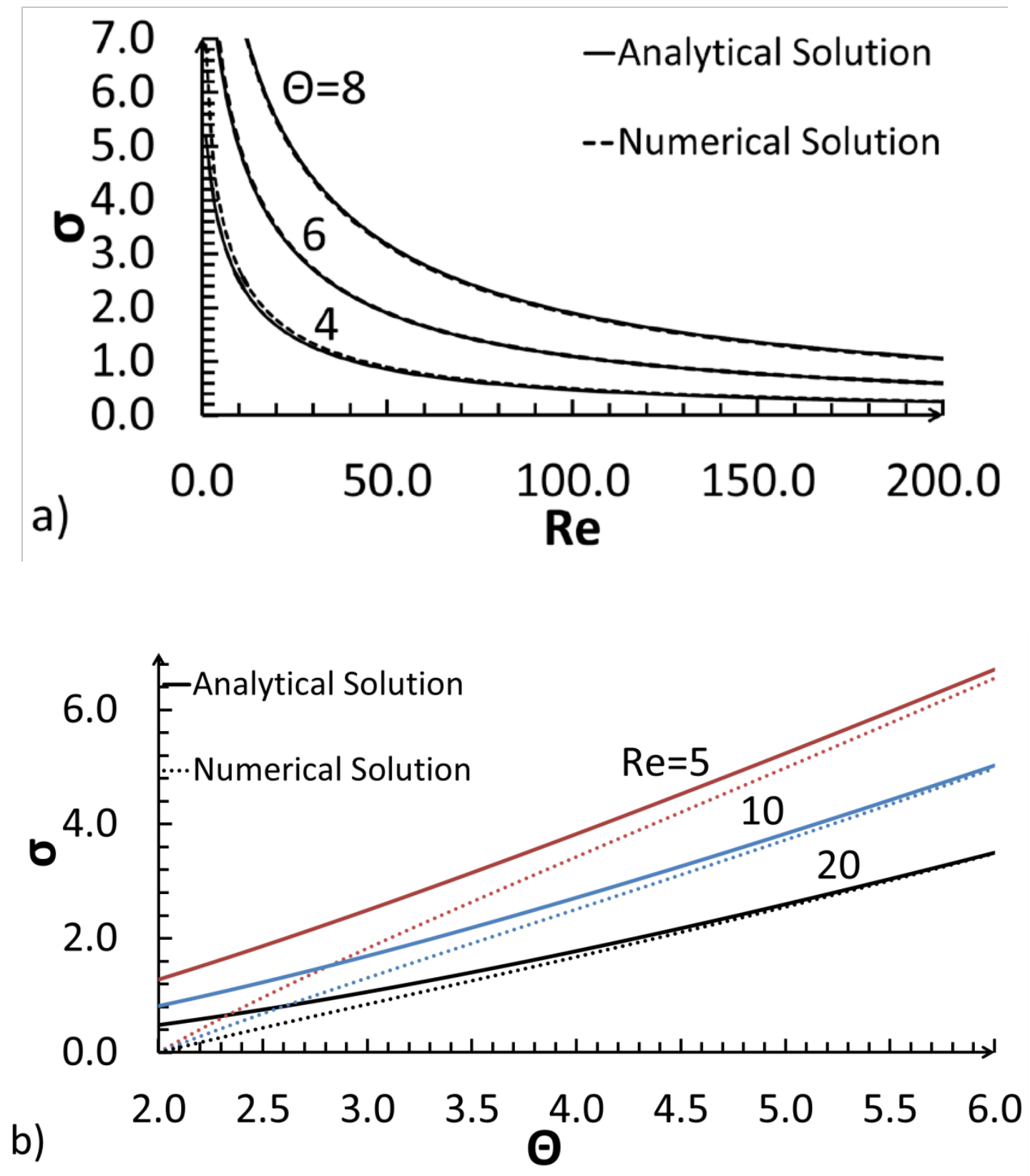

FIG. 2.9. $\sigma$ versus $\operatorname{Re}$ at $\Theta=$ 4; 6; 8 (a); and versus $\Theta$ at $\operatorname{Re}=$ 5; 10; 20 (b). Equations (2.15) and (2.17) are shown by dashed and solid lines, respectively 
Nevertheless, while very good agreement between Eqs. (2.15) and (2.17) is evident at large $\Theta$ and Re, the discrepancy between them gets stronger with the decrease in these parameters and thereby deteriorates the quantitative predictability of the theory; while Eq. (2.17) yields a nearparabolic dependence of $\sigma$ versus $\Theta$, according to Eq. (2.15), $\sigma \rightarrow 0$ when $\Theta \rightarrow 2$. Again, the latter trend reduces the gap between the present theory and the formulation on steady flame propagation of Akkerman et al., 2010 yielding that a flame surely accelerate in an axisymmetric configuration with one end closed if $\Theta>2$.

Again, the quantitative analysis of such a discrepancy is implemented, see Figs. 2.10 and 11. Specifically, Fig. 2.10a shows the relative error, Eq. (2.11), versus $\Theta$ at various Re, while Fig. 2.10b demonstrates the relative error versus Re at several fixed $\Theta$.

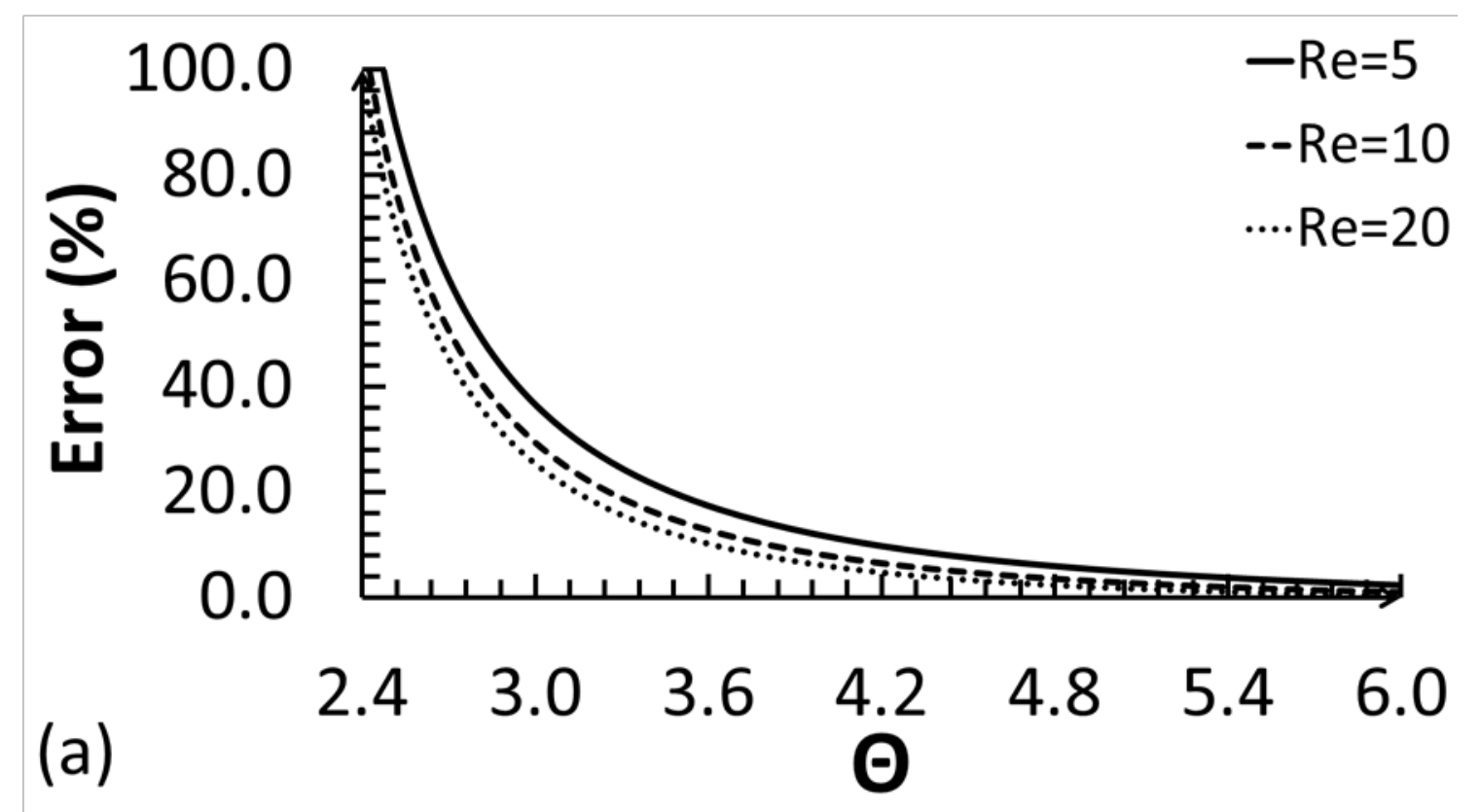




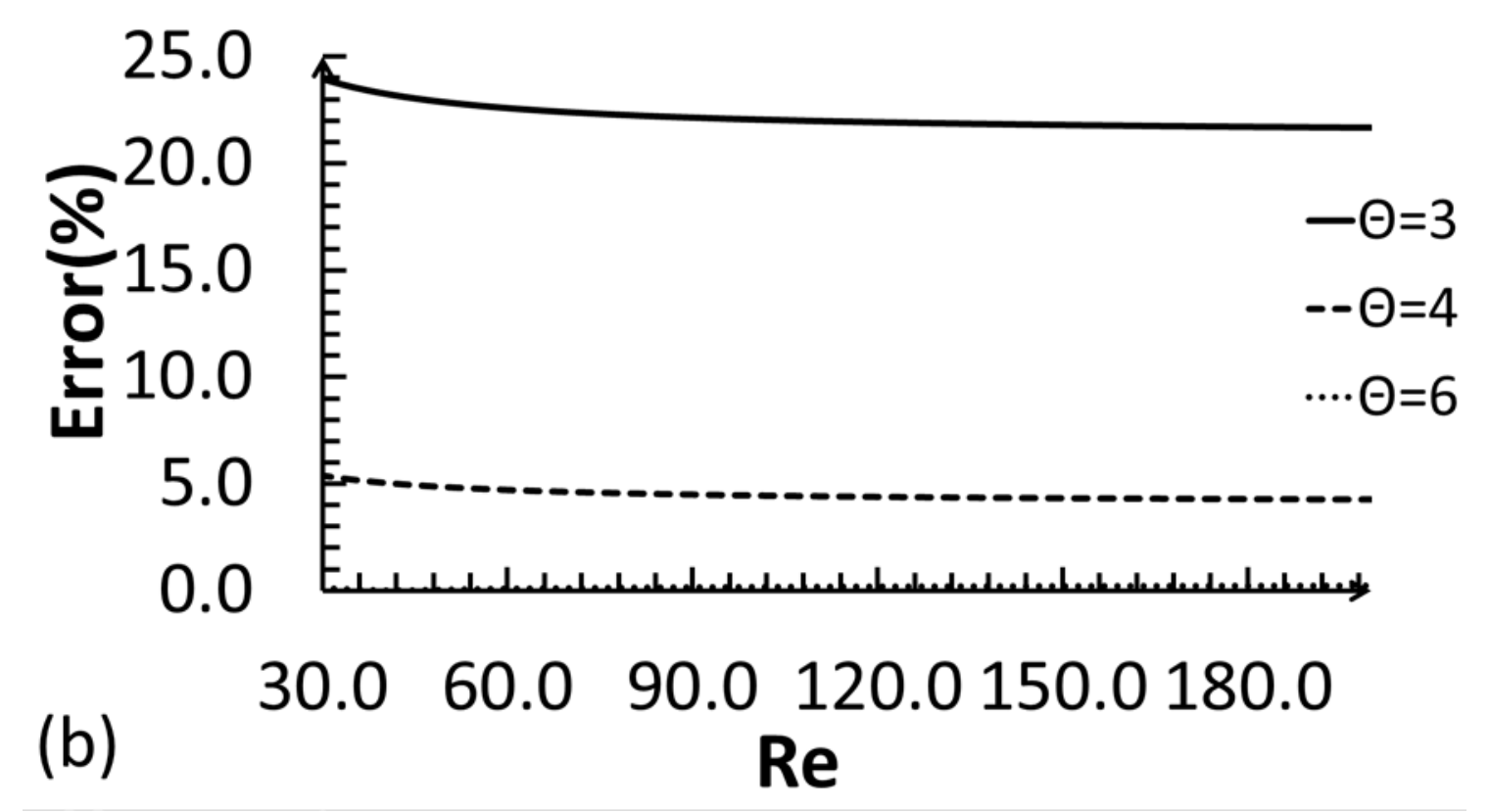

FIG. 2.10. The relative error (in \%), Eq. (2.11), versus $\Theta$ at $\operatorname{Re}=5,10,20$, shown by solid, dashed and dotted lines, respectively (a); and versus Re at $\Theta=3,4,6$ shown by solid, dashed and dotted lines, respectively (b)

It is clearly seen that the error reduces with the increase in $\Theta$ and/or Re. Figure 2.11 quantifies the intrinsic limitations of the theory. The limitations are demonstrated by means of the validity domains in the relevant Re- $\Theta$ diagram. Namely, Fig. 2.11 represents the contour plot analysis in the Re- $\Theta$ space. The error isolines show the accuracy domains (and thereby the intrinsic limitations of the theory). It is clearly seen that the error is very low when both thermal expansion and the flame propagation Reynolds numbers are high, but the situation substantially deteriorates with the decrease in Re and/or $\Theta$. Specifically, the relative error, Eq. (2.11), is less than $1 \%$ in the top right corner of Fig. 2.11; this is the domain where the $1^{\text {st }}$ order analytical approximation, Eq. (2.17), works pretty well. However, the error keeps increasing towards the left bottom corner, exceeding 50\% therein. Obviously, the quantitative applicability of Eq. (2.17) is doubtful in that case. 


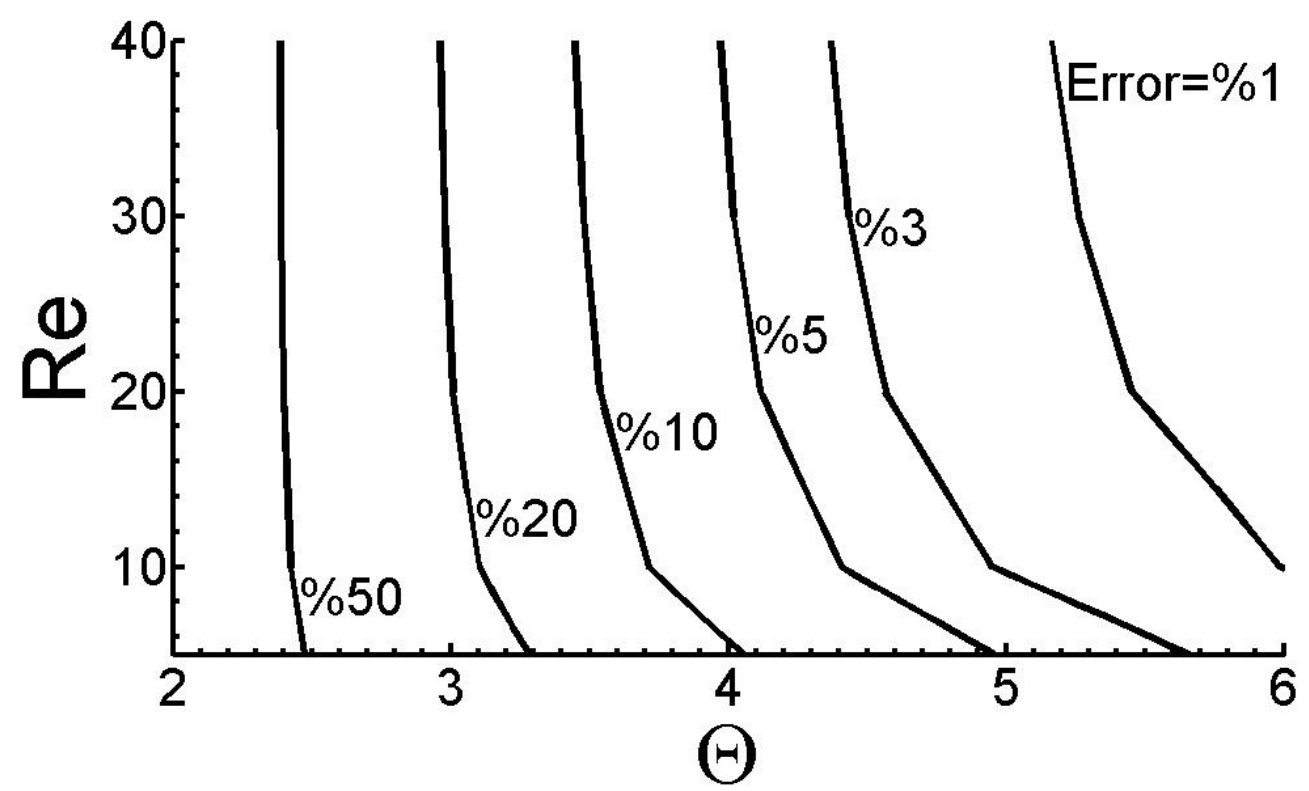

FIG. 2.11. Contour scheme (the error isolines (in \%)) demonstration in the Re- $\Theta$ diagram

\subsection{Conclusions}

In the present paper the analytical formulations for the flame acceleration in $2 \mathrm{D}$ channels (Bychkov et al., 2005) and cylindrical tubes (Akkerman et al., 2006) were revisited, and their intrinsic limitations were determined. It is shown that while the original theory (Bychkov et al., 2005) predicts the main characteristics of the flame acceleration very well at large and Re, the accuracy deteriorates at other condition. This is substantiated, in particular, by the error contours in the Re- $\Theta$ diagram of Fig. 2.5. This $2 \mathrm{D}$ trend was actually expected. In contrast, the $0^{\text {th }}$ order approximation of the cylindrical-axisymmetric configuration (Akkerman et al., 2006), Eq. (2.16), appeared to be inaccurate, even for realistically large Re and/or $\Theta$. At the same time, the $1^{\text {st }}$ order approximation, Eq. (2.17), is pretty accurate for a wide range of parameters. 


\section{Chapter 3: Computational Platform}

A fully-compressible, finite-volume Navier-Stokes “in-house” solver of Dr. Akkerman’s group at West Virginia University has been used as the major computational platform of this work. The embryo of this code was developed originally by Dr. Lars-Erik Eriksson at Volvo Aero Company (Goteborg, Sweden), and it was subsequently updated, comprehensively, by several research groups, including the group of Prof. M. Liberman at Uppsala University as well as that of Prof. V. Bychkov at Umea University, both in Sweden, and eventually the group of Dr. Akkerman. One of the active developers of this computational platform is Dr. Damir Valiev, who is currently affiliated with Sandia National Laboratories and Princeton University.

The code solves the complete set of the hydrodynamic and combustion equations including transport processes (diffusion, viscosity, heat conduction) and the Arrhenius chemical kinetics. The numerical scheme is second order accurate in time, fourth order in space for the convective terms, and second order in space for the diffusive term. The solver is robust and accurate, having been successfully utilized in numerous aero-acoustic and combustion applications (Erikkson, 1987; Erikkson, 1995; Anderson et al., 2005; Wollblad et al., 2006). It is adapted for parallel computations and available in 2D (Cartesian and cylindrical axisymmetric) version, as well as fully 3D Cartesian version, with a self-adaptive structured computational grid employed that makes the code perfect, in particular, for fundamental studies of flame hydrodynamics in combustion tubes, chambers as well as for outwardly-propagating flames in free space.

\subsection{Governing equations}

In the general form, the complete set of the governing equations of compressible hydrodynamic and combustion include: the continuity equation, 


$$
\frac{\partial \rho}{\partial t}+\frac{1}{r^{\beta}} \frac{\partial}{\partial r}\left(r^{\beta} \rho u_{r}\right)+\frac{\partial}{\partial z}\left(\rho u_{z}\right)=0
$$

the Navier-Stokes (momentum) equation,

$$
\begin{aligned}
& \frac{\partial}{\partial t}\left(\rho u_{z}\right)+\frac{1}{r^{\beta}} \frac{\partial}{\partial r}\left[r^{\beta}\left(\rho u_{z} u_{r}-\zeta_{z, r}\right)\right]+\frac{\partial}{\partial z}\left(\rho u_{z}^{2}-\zeta_{z, z}\right)+\frac{\partial P}{\partial r}+\Psi_{\beta}=0 \\
& \frac{\partial}{\partial t}\left(\rho u_{r}\right)+\frac{1}{r^{\beta}} \frac{\partial}{\partial r}\left[r^{\beta}\left(\rho u_{r}^{2}-\zeta_{r, r}\right)\right]+\frac{\partial}{\partial z}\left(\rho u_{r} u_{z}-\zeta_{r, z}\right)+\frac{\partial P}{\partial r}+\Psi_{\beta}=0,
\end{aligned}
$$

the energy balance equation,

$$
\begin{aligned}
\frac{\partial \varepsilon}{\partial t}+\frac{1}{r^{\beta}} \frac{\partial}{\partial r}\left[r^{\beta}\left((\varepsilon+P) u_{r}-\zeta_{r, r} u_{r}-\zeta_{r, z} u_{z}+q_{r}\right)\right]+ \\
\frac{\partial}{\partial z}\left[(\varepsilon+P) u_{z}-\zeta_{z, z} u_{z}-\zeta_{r, z} u_{r}+q_{z}\right]=0
\end{aligned}
$$

and the species equation,

$$
\frac{\partial}{\partial t}(\rho Y)+\frac{1}{r^{\gamma}} \frac{\partial}{\partial r}\left[r^{\gamma}\left(\rho u_{i} Y-\frac{\mu}{\mathrm{Sc}} \frac{\partial Y}{\partial r}\right)\right]+\frac{\partial}{\partial z}\left(\rho u_{z} Y-\frac{\mu}{\mathrm{Sc}} \frac{\partial Y}{\partial z}\right)=-\frac{\rho Y}{\tau_{R}} \exp \left(-E_{a} / R_{p} T\right)
$$

where $\beta=0$ and 1 for $2 \mathrm{D}$ and axisymmetric geometries respectively,

$$
\varepsilon=\rho\left(Q Y+C_{V} T\right)+\frac{\rho}{2}\left(u_{z}^{2}+u_{r}^{2}\right),
$$

is the total energy per unit volume, $Y$ the mass fraction of the fuel, $Q$ the energy release from the reaction, and $C_{V}$ the heat capacity at constant volume. The energy diffusion vector $q_{i}$ is given by

$$
\begin{aligned}
& q_{r}=-\mu\left(\frac{C_{P}}{\operatorname{Pr}} \frac{\partial T}{\partial r}+\frac{Q}{\text { Sc }} \frac{\partial Y}{\partial r}\right), \\
& q_{z}=-\mu\left(\frac{C_{P}}{\operatorname{Pr}} \frac{\partial T}{\partial z}+\frac{Q}{\operatorname{Sc}} \frac{\partial Y}{\partial z}\right) .
\end{aligned}
$$

The stress tensor $\zeta_{i, j}$ takes the form 


$$
\zeta_{i, j}=\mu\left(\frac{\partial u_{i}}{\partial x_{j}}+\frac{\partial u_{j}}{\partial x_{i}}-\frac{2}{3} \frac{\partial u_{k}}{\partial x_{k}} \delta_{i, j}\right)
$$

in the $2 \mathrm{D}$ configuration ( $\beta=0$ ), while in the axisymmetric geometry $(\beta=1)$ it reads

$$
\begin{aligned}
& \zeta_{r, r}=\frac{2 \mu}{3}\left(2 \frac{\partial u_{r}}{\partial r}-\frac{\partial u_{z}}{\partial z}-\frac{u_{r}}{r}\right), \\
& \zeta_{z, z}=\frac{2 \mu}{3}\left(2 \frac{\partial u_{z}}{\partial z}-\frac{\partial u_{r}}{\partial r}-\frac{u_{r}}{r}\right), \\
& \zeta_{r, z}=\mu\left(\frac{\partial u_{r}}{\partial z}+\frac{\partial u_{z}}{\partial r}\right) .
\end{aligned}
$$

Finally, the last term in Eq. (3.2) takes the form

$$
\psi_{\beta}=\frac{2 \mu}{3}\left(2 \frac{u_{r}}{r}-\frac{\partial u_{r}}{\partial r}-\frac{\partial u_{z}}{\partial z}\right)
$$

if $\beta=1$, and $\psi_{\beta}=0$ if $\beta=0$. Here $\mu$ is the dynamic viscosity, Pr and Sc the Prandtl and Schmidt numbers, respectively. Unburned and burned mixtures are assumed to be ideal twoatomic gases and have the same molecular weights $m_{f}=m_{b}=m$. Then the specific heats are $C_{V}=5 R_{p} / 2 m, C_{P} \equiv C_{V}+R_{p}=7 R_{p} / 2 m$, and the equation of state reads

$$
P=\rho R_{p} T / m
$$

where $R_{P} \approx 8.314 \mathrm{~J} /(\mathrm{mol} \cdot \mathrm{K})$ is the universal gas constant. A one-step irreversible Arrhenius reaction of the $1^{\text {st }}$ order is typically employed (though the reaction order may vary), with the activation energy $E_{a}$ and frequency factor corresponding to a characteristic time $\tau_{R}$. The factor $\tau_{R}$ is adjusted to obtain a particular value of the unstretched laminar flame speed $U_{f}$ by solving the associated eigenvalue problem. In other words, the flame velocity is almost fully determined by the set of chemical parameters of the fuel: $E_{a}, \tau_{R}, Q$. Compressibility is characterized by the initial Mach number coupled to the planar flame speed, $M a \equiv U_{f} / c_{S}$. For typical hydrocarbon 
deflagrations it is pretty small, $0.001 \sim 0.01$. In that case, the flow is almost isobaric and thermal expansion is coupled to the energy release in the burning process as follows

$$
\Theta=T_{b} / T_{f}=1+Q / C_{P} T_{f} .
$$

The flame thickness is conventionally defined as

$$
L_{f} \equiv \frac{\mu}{\operatorname{Pr} \rho_{f} U_{f}} \equiv \frac{D_{t h}}{U_{f}},
$$

where $D_{\text {th }}$ is the thermal diffusivity and Pr is the Prandtl number. It is noted that the quantity (3.15) is a characteristic parameter of the problem of length dimension, while the real size of the combustion zone can be several times wider. In a certain sense, $L_{f}$ characterizes the active reaction zone rather than the real flame thickness, see Fig. 3.1.

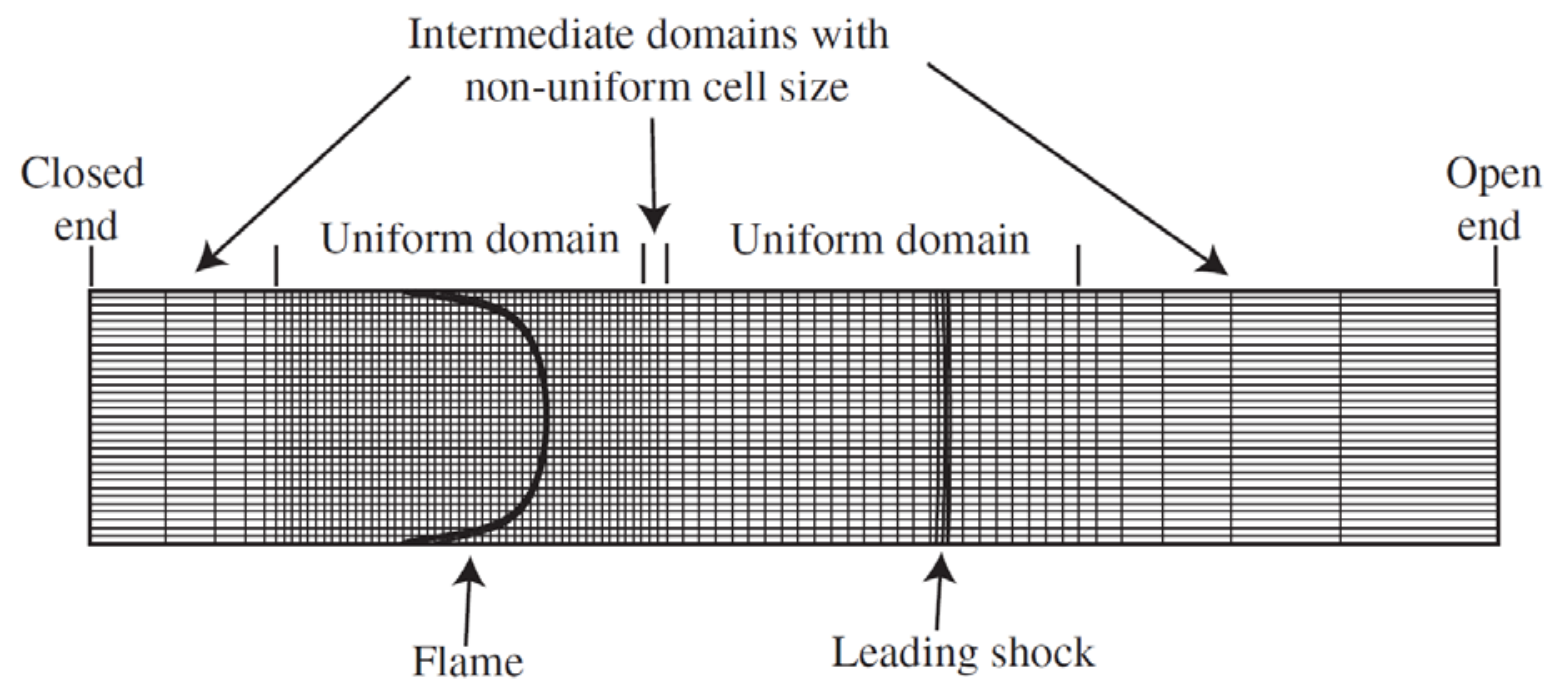

FIG. 3.1. The sketch of the grid with variable resolution used in numerical simulations

It is nevertheless noted that $L_{f}$ is a key parameter for simulations. First of all, it is readily related to the flame propagation Reynolds number, $\operatorname{Re}=R /\left(L_{f} \operatorname{Pr}\right)$, which is an important parameter itself. As a matter of fact, this is a characteristic transport scale, and it has sense even in the cold, 
non-reacting flow. In addition, the resolution abilities of the solver are given by $L_{f}$ : from 2 to 5 grid points have to be taken per $L_{f}$ to imitate the combustion process properly. The current version of the solver allows taking up to 5-10 million grid points (cells) on modern clusters, while RAM problems may appear at larger mesh sizes. Therefore, one can readily determine the maximal scale that can be resolved for a particular computational configuration.

Mesh with variable resolution is used in order to take into account the growing distances between the tube end, the accelerating flame and the pressure wave, and to resolve both chemical and hydrodynamic spatial scales. Typical computation time for one simulation required up to 104 CPU-hours, hence implying the need for extensive parallel calculations. A rectangular grid with the grid walls parallel to the coordinate axes was used. The sketch of the calculation mesh used in the simulations of flame acceleration from the closed tube end is shown in Fig. 3.1. To perform all the calculations in a reasonable time, the grid spacing non-uniform along the z-axis with the zones of fine grid around the flame and leading shock fronts were established. For the majority of the simulation runs, the grid size in the z-direction was $0.25 L_{f}$ and $0.5 L_{f}$ in the domains of the flame and leading pressure wave, respectively, which allowed resolution of the flame and waves. Outside the region of fine grid the mesh size increased gradually with $2 \%$ change in size between the neighboring cells. In order to keep the flame and pressure waves in the zone of fine grid, the periodical mesh reconstruction during the simulation run (Valiev et al., 2008) were employed. Third-order splines were used for interpolation of the flow variables during periodic grid reconstruction to preserve the second order accuracy of the numerical scheme. 


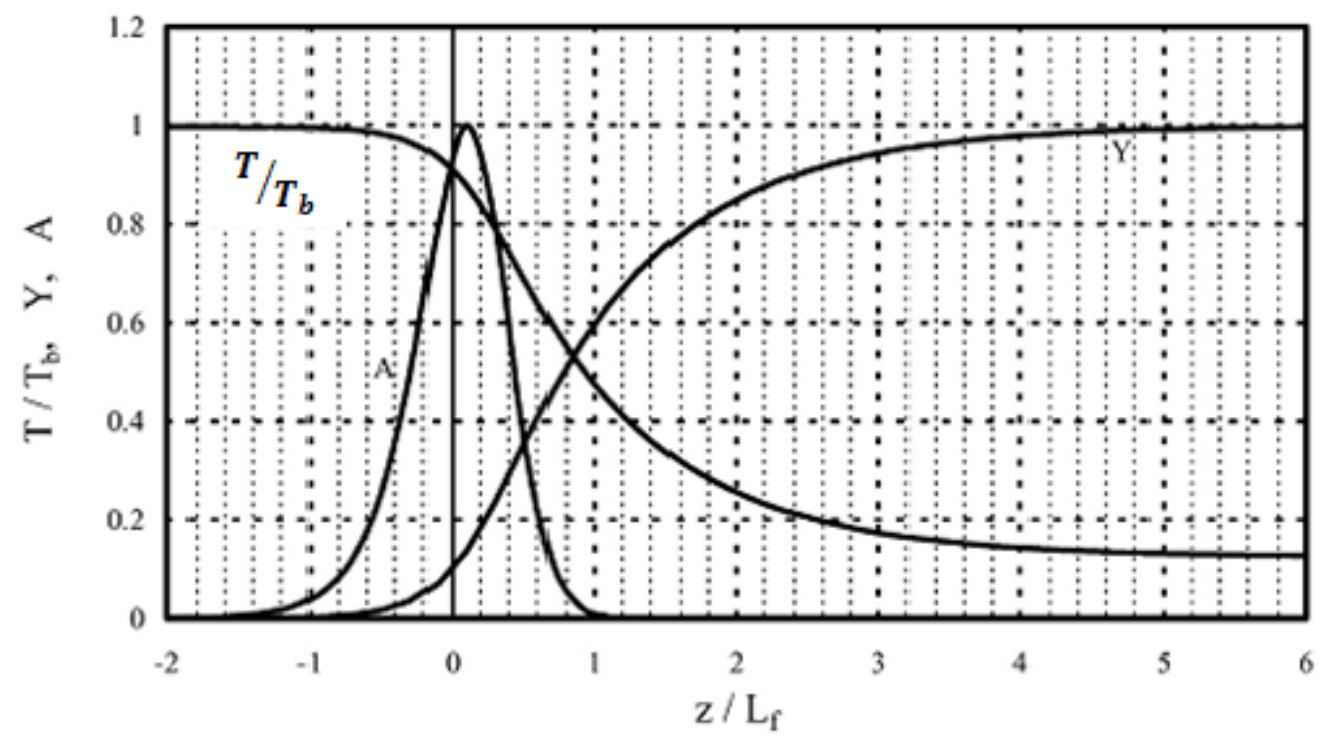

FIG. 3.2. Profiles of the scaled temperature $T / T \mathrm{~b}$, the mass fraction $Y$, and the reaction rate $A \propto(\rho Y / \tau \mathrm{r}) \times$ $\exp (-E a / R p T b)$, scaled by its maximal value

It is emphasized that the Reynolds number related to the flow of the fuel mixture considerably differs from that related to the flame propagation. Indeed, one has

$$
\operatorname{Re}_{\text {flow }} \equiv \frac{2 R\left\langle u_{z}\right\rangle}{v}=\frac{2(\Theta-1) U_{w} R}{v}=\frac{2(\Theta-1)}{\operatorname{Pr}} \frac{U_{w}}{U_{f}} \frac{R}{L_{f}}=2(\Theta-1) \Omega_{w} \operatorname{Re} .
$$

Consequently the flow Reynolds number can exceed the flame Reynolds number by orders of magnitude, and while the former may reach the turbulent threshold, say $10^{3}-10^{4}$, the latter is still quite small $10-10^{2}$ to be resolved in direct numerical simulations. Thus this solver can simulate turbulent combustion as well. (Akkerman et al., 2007; Akkerman et al., 2008).

\subsection{Numerical Scheme}

For the sake of brevity, the description will focus on the 2D configuration only. Nevertheless, it can readily be modified for an axisymmetric configuration, as well as for a 3D Cartesian one. The code is based on the cell-centred finite-volume scheme, which is robust for modelling of different kinds of complex hydrodynamic flows (Eriksson, 1987), as has been validated, in 
particular, by Travnikov et al. 2002 by means of the known exact solutions of the classical hydrodynamic problems. Specifically, each of the governing equations (3.1) - (3.4) is written in conservation form as

$$
\frac{\partial G}{\partial t}+\frac{\partial E_{G}}{\partial x}+\frac{\partial F_{G}}{\partial z}=H_{G}
$$

where $G$ stands for any of the variables $\rho, \rho u_{x}, \rho u_{z}, \rho e+\frac{1}{2} \rho\left(u_{x}^{2}+u_{z}^{2}\right), \rho Y$; while $E_{G}, F_{G}$ denotes the corresponding fluxes and $H_{G}$ gives a source term. The cell-centred finite-volume spatial discretization is achieved by integrating the conservation law in the form (3.9) over a given grid cell. As an example, the result of integration for an interior cell with index $i, j$ is illustrated here. It is assumed that purely integer indexes (i,j) denote the grid cells, mixed fractional-integer indexes stand for the cell walls, and purely fractional indexes denote the grid nodes. Within this notation, one can write:

$$
\left.\frac{d}{d t} \bar{G}_{i, j}+\left(E_{G}\right)_{i+1 / 2, j}-\left(E_{G}\right)_{i-1 / 2, j}+\left(F_{G}\right)_{i, j+1 / 2}-\left(F_{G}\right)_{i, j-1 / 2}=\overline{(H}_{G}\right)_{i, j},
$$

where

$$
\begin{aligned}
&\left.\bar{G}_{i, j}=\frac{1}{\alpha_{i, j}} \int_{\Omega_{i, j}} G d S, \overline{(H}_{G}\right)_{i, j}= \frac{1}{\alpha_{i, j}} \int_{\Omega_{i, j}} H d S, \alpha_{i, j}=\int_{\Omega_{i, j}} d S, \\
&\left(E_{G}\right)_{i+\frac{1}{2}, j}=\int_{B_{i+\frac{1}{2}, j}}\left(E_{G} n_{x}+F_{G} n_{z}\right) d l, \\
&\left(F_{G}\right)_{i, j+1 / 2}=\int_{B_{i, j+1 / 2}}\left(E_{G} n_{x}+F_{G} n_{z}\right) d l,
\end{aligned}
$$

$\Omega_{i, j}$ is the grid cell (i, j), $B_{i+1 / 2, j}$ and $B_{i, j+1 / 2}$ are the cell walls between the current cell (i, j) and the cells $(\mathrm{i}+1, \mathrm{j})$ and $(\mathrm{i}, \mathrm{j}+1)$ respectively, $n=\left(n_{x}, n_{z}\right)$ is the normal to the corresponding cell wall. By choosing the cell-averages of the state vector $\bar{G}_{i, j}$ as the unknowns of the discretized problem and introducing approximations of the fluxes $\left(\overline{E_{G}}\right)_{i+1 / 2, j}$ and $\left(\overline{F_{G}}\right)_{i, j+1 / 2}$ and the cell 
averaged source vector $\left(\overline{H_{G}}\right)_{i, j+1 / 2}$ in terms of these unknowns, the final spatial discretization of (3.9) can be obtained.

A key feature of the cell-centred finite volume discretization of (3.9) given by (3.10) is the numerical approximation of the fluxes $\left(\overline{E_{G}}\right)_{i+1 / 2, j}$ and $\left(\overline{F_{G}}\right)_{i, j+1 / 2}$ in terms of the cell averages $\bar{G}_{i, j}$. The usual approach is to treat the convective flux approximations and the diffusive flux ones separately due to the different nature of these fluxes. For the convective fluxes, a characteristicupwind flux scheme (Eriksson, 1987), where the propagation directions of the various characteristic variables control a user-given degree of up-winding, is employed. Here it turns out to be advantageous to work with the hydro-dynamical variables $\rho, u_{x}, u_{z}, P, Y$, instead of the conservative variables in the state vector $\bar{G}_{i, j}$. The numerical errors introduced by using this approximation are of the second order in grid spacing assuming a smooth solution. For problems where all spatial scales are adequately resolved in the computational grid, an extremely small amount of up-winding may be used, giving an almost centred scheme with minimal numerical dissipation and dispersion.

\subsection{Principles of setting the initial conditions}

\section{a) Planar flame front ignition}

The easiest but feasible way to employ the initial conditions for an initially planar premixed flame front is to approximate it by the classical Zeldochich-Frank-Kamenetsky (ZFK) solution (Bychkov and Liberman, 2000). In the reference frame co-moving with the flame front it reads

$$
\frac{T(z)}{T_{f}}= \begin{cases}1+(\Theta-1) \exp \left(-z / L_{f}\right), & z \geq 0 \\ \Theta, & z<0\end{cases}
$$




$$
\frac{P(z)-P_{f}}{\rho_{f} S_{L}^{2}}= \begin{cases}\left(\frac{4}{3} \operatorname{Pr}-1\right)(\Theta-1) \exp \left(-z / L_{f}\right), & z \geq 0 \\ (\Theta-1), & z<0\end{cases}
$$

with

$$
\frac{u_{z}(z)}{S_{L}}=\frac{\rho_{f}}{\rho(z)}=\frac{T}{T_{f}}, \quad Y(z)=\frac{\Theta-T / T_{f}}{\Theta-1} .
$$

\section{b) Hemispherical flame front ignition}

At the open tube end, the non-reflecting boundary conditions are applied. As initial conditions, a hemi-spherical (hemi-circular) version of the ZFK solution is used, namely

$$
\begin{aligned}
& T=T_{f}+\left(T_{b}-T_{f}\right) \exp \left(-\sqrt{r^{2}+z^{2}} / L_{f}\right), \quad \text { if } \quad z^{2}+x^{2}<r_{f}^{2}, \\
& T=\Theta T_{f}, \quad \text { if } \quad z^{2}+x^{2}>r_{f}^{2}, \\
& Y=\left(T_{b}-T\right) /\left(T_{b}-T_{f}\right), P=P_{f}, u_{x}=0, u_{z}=0 .
\end{aligned}
$$

Here $r_{f}$ is the radius of initial flame ball at the closed end of the tube. The finite initial radius of the flame ball is equivalent to a time shift, which required proper adjustments when comparing the theory and numerical simulations. When necessary, the numerical solution in time is shifted in order to have the theory and the modeling results starting from the same point. 


\section{Chapter 4: Effect of Thermal Expansion on Flame Propagation in}

\section{Channels with Nonslip Walls: Numerical and Analytical}

\section{Consideration}

\subsection{Motivation}

To begin with, it is pointed out that the theories (Bychkov et al., 2005; Akkerman et al., 2006) include a set of assumptions such as the large Reynolds number related to flame propagation, as well as the large thermal expansion coefficient in the burning process, which in turn leads to the intrinsic limitations of the theory. In Chapter 2, these limitations have been determined, and thereby validity domains of the Bychkov approximation within a $\Theta$ - Re diagram were clearly underlined. Furthermore, the error analysis related to the intrinsic assumptions and accuracy of the developed theory was also performed.

The main purpose of the present component of this thesis is to substantiate the validity domains obtained in Chapter 2, in particular, as well as the formulation (Bychkov et al., 2005), in general, by usage of comprehensive direct numerical simulations for the variety of parameters, in particular, $\Theta$ and Re. The numerical simulations are performed for the complete set of combustion equations including thermal conduction, viscosity, diffusion and chemical kinetics with one-step Arrhenius reaction. It is demonstrated that while the simulations fit the theoretical prediction for large $\Theta$, a decrease in $\Theta$ and a variation of Re lead to the deviation from the exponential state of acceleration. The planar flame speed is taken to be $U_{\mathrm{f}}=30-40 \mathrm{~cm} / \mathrm{s}$, providing a realistically low flame propagation Mach number, $\sim 10^{-3}$, and the thermal expansion in the burning process was taken in the range of $\Theta=4 \sim 12$, which fits typical hydrocarbon 
combustion. It is recalled that the solver and the numerical method are described in Chapter 3 as well as in (Bychkov et al., 2005; Akkerman et al.,2006; Akkerman et al., 2010; Bychkov et al., 2010 and Valiev et al., 2013).

\subsection{Computational Results and Discussion}

Here computational results are presented and compared to the asymptotical prediction of (Bychkov et al., 2005), Eq. (2.25), as well as to the numerical solution to Eq. (2.23) representing the so-called "semi-analytical (numerical)” approach of Chapter 2. Specifically, it is recalled that one more purpose of the present part is to reconcile the asymptotical analysis (Bychkov et al., 2005) and the recent work (Demirgok and Akkerman, 2013), described in Chapter 2, that underlined the intrinsic limitations of the theory (Bychkov et al., 2005). Specially, by solving Eq. (2.23) computationally, it is determined whether the asymptotic (2.25) works reasonably for a given set of parameters, and if it works, what the effect of thermal expansion on the flame propagation is, and how the limitations within a $\Theta$-Re diagram look; see (Demirgok and Akkerman, 2013) for details.

Figure 4.1 shows the evolution of the locus of the flame tip for the variety of flame propagation Reynolds numbers and expansion coefficients $(\Theta \geq 8)$ and flame propagation Reynolds numbers $\operatorname{Re}=10 \sim 50$. Specifically, Figs. 4.1 (a-d) correspond to the fixed $\Theta=12$ (a), 10 (b), 9 (c), and 8 (d), with various Re in each plot; while Figs. 4.1 (e, f) correspond to the fixed $R e=20$ (e) and $\operatorname{Re}=10$ (f), with various $\Theta=6,8,10,12$ in each plot. The solid lines represent the direct numerical simulation of the present work, while the dashed lines show the fit for the exponential trend suggested by the theory (Bychkov et al., 2005). 
In Figure 4.1 (a-d) one observes very good agreement between the computational and theoretical results. Indeed, look at the respective solid lines, representing direct numerical simulations, and the dashed ones, showing an exponential fit suggested by the theory. It is clearly shown that the respective results coincidence and overlap each other. Also, it can be stated that a flame propagates faster in a narrower tube. Indeed, this is seen in Fig.4.1a, where two different tubes are compared at fixed $\Theta=12$, with the flame propagation Reynolds numbers being 25, 15 and 10, respectively. One can observe that the solution with lower Re exceeds that at higher Re for the same scaled time instant. Therefore, the flame acceleration rate decreases with the tube radius.

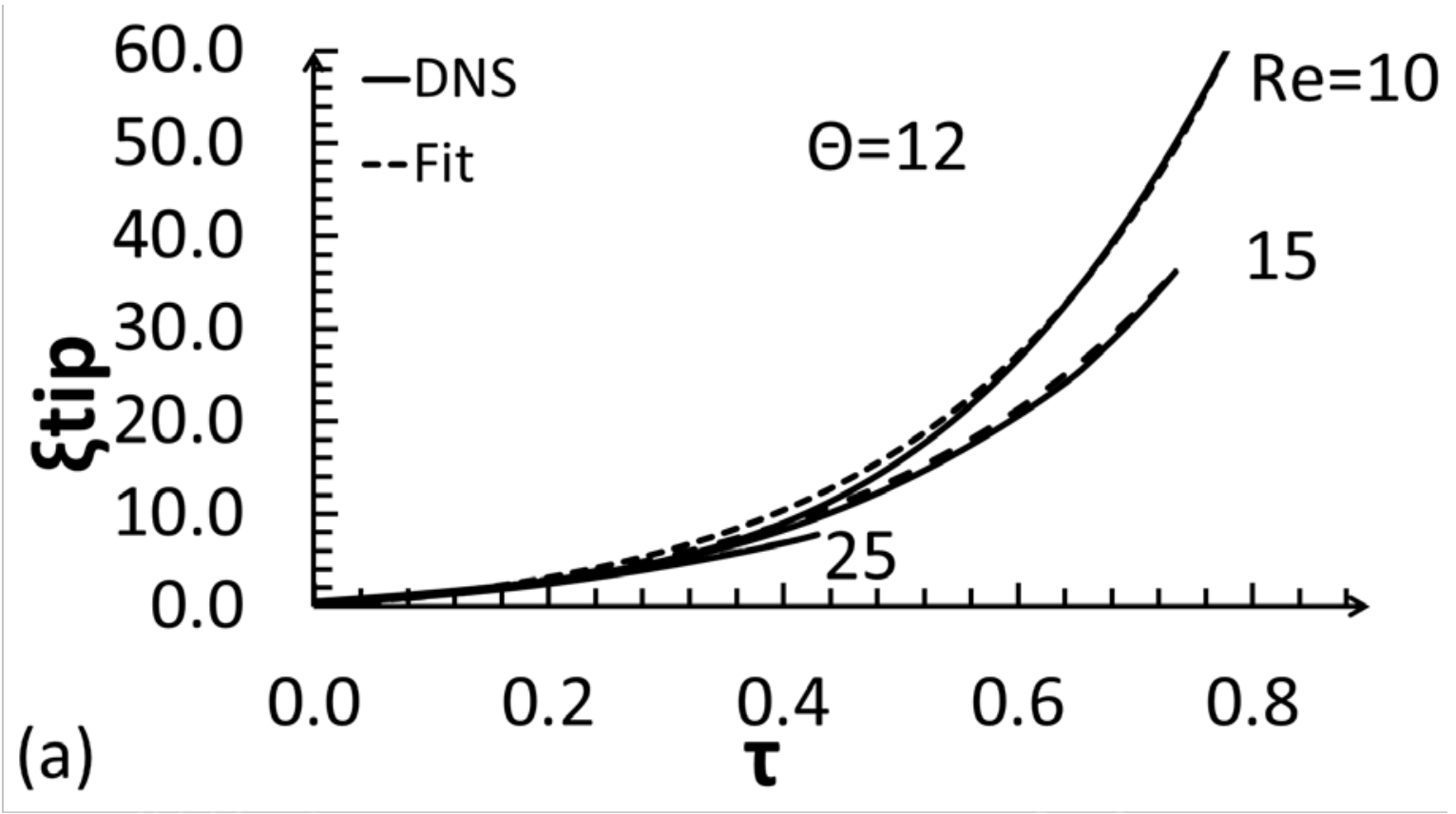



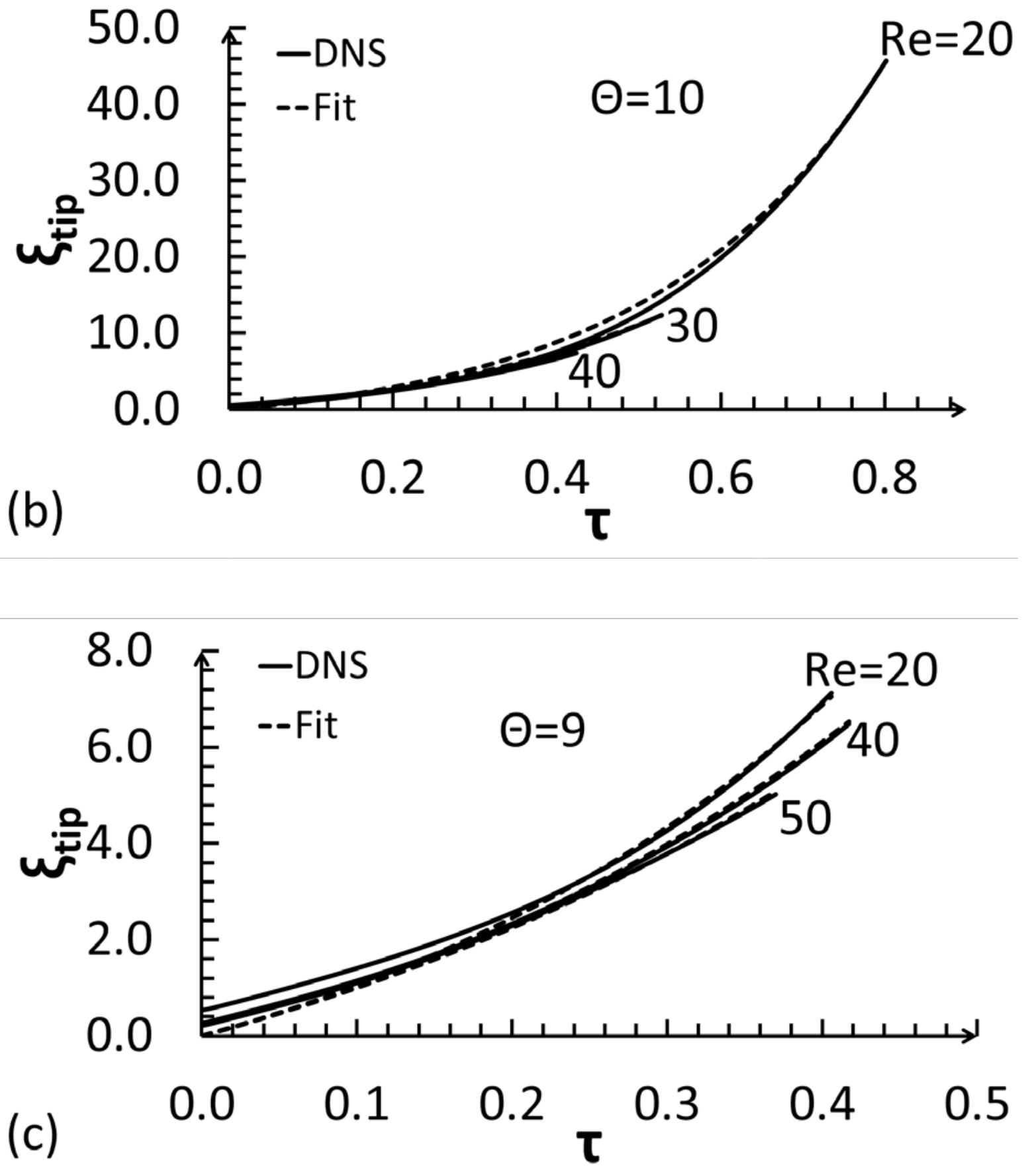

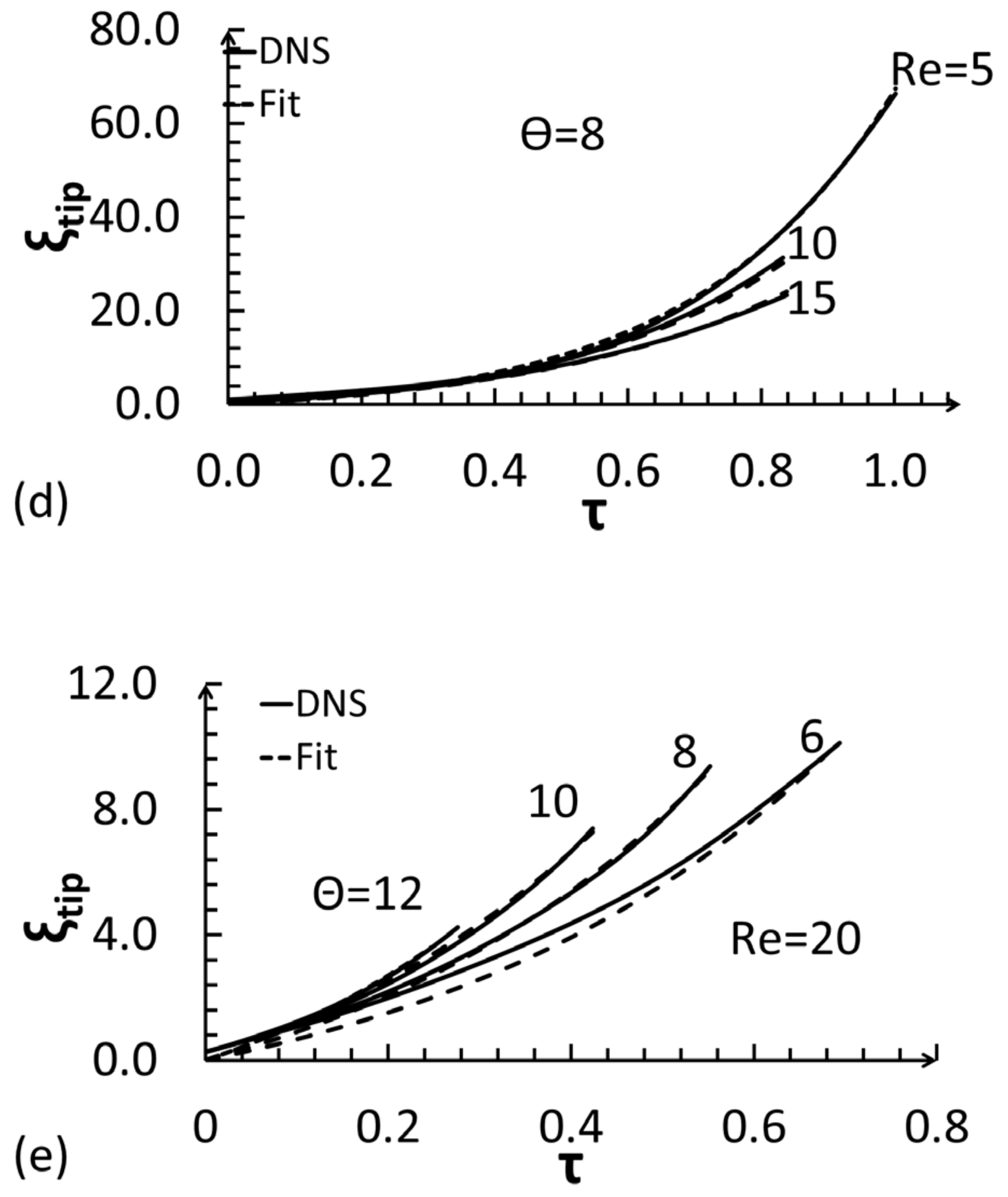


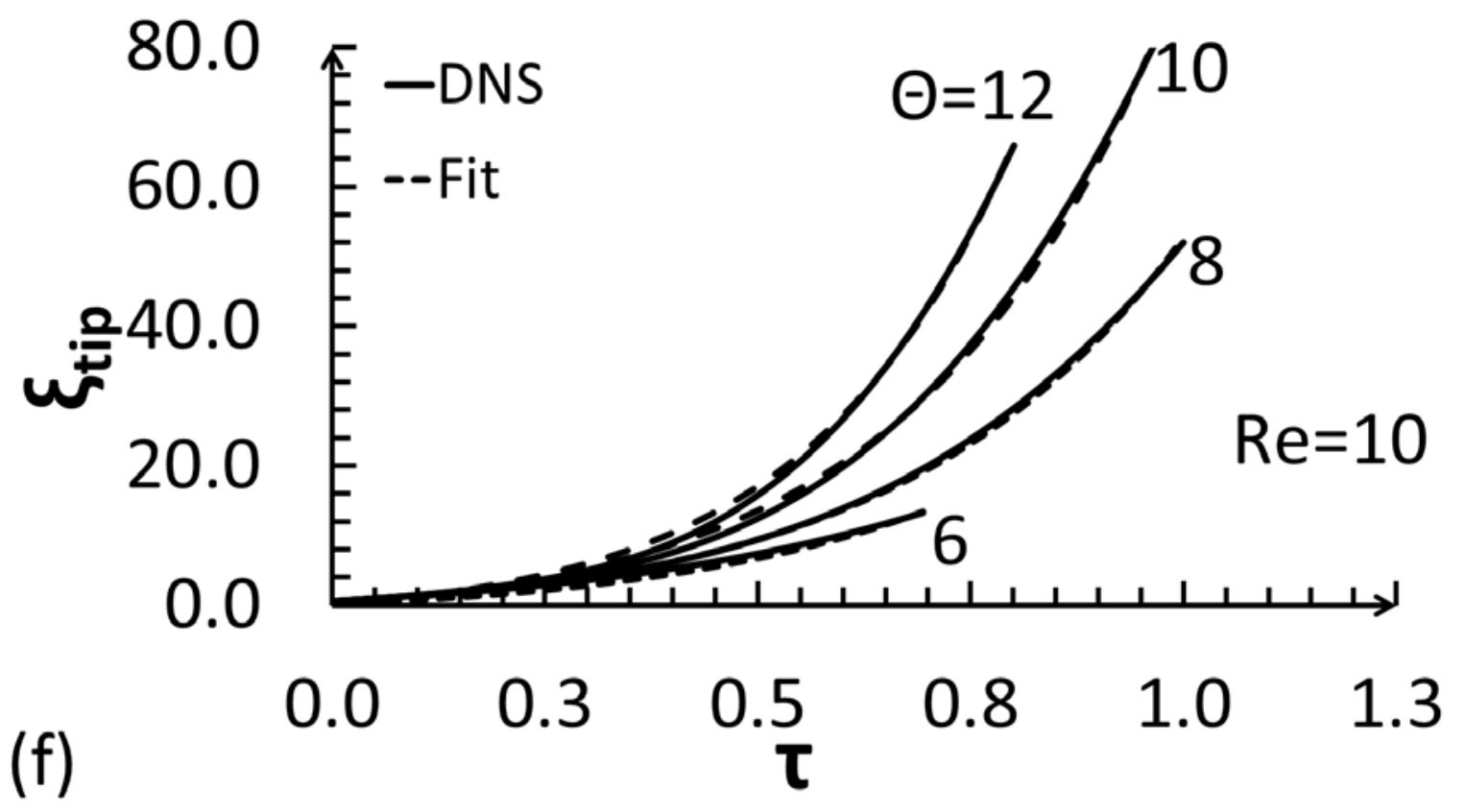

FIG. 4.1. The scaled flame tip position $\xi_{\text {tip }}$ versus the scaled time $\tau$ at fixed thermal expansion coefficients, namely $\Theta=12$ (a), $\Theta=10$ (b), $\Theta=9$ (c), and $\Theta=8$ (d), with various flame propagation Reynolds numbers in each plot and the scaled flame tip position $\xi_{\text {tip }}$ versus the scaled time $\tau$ at thermal expansion coefficients $\Theta=12,10,8$ and 6 and fixed propagation Reynolds number $\operatorname{Re}=20$ (e) $\operatorname{Re}=10$

In a similar manner, in order to investigate the thermal expansion effects on the flame propagation, in Fig. 4.1 (e-f) the evolution of the locus of the flame tip is shown for the variety of thermal expansion coefficients, $\Theta=6-12$, with a fixed flame propagation Reynolds number in each plot, namely $\mathrm{Re}=20$ and 10 in Fig. 4.1 (e) and 4.1 (f), respectively. Again, one can see very good agreement between the computational and theoretical results in Fig. 4.1 (e-f). Indeed, the respective solid lines, representing the DNS' results, and the dashed ones, showing an exponential fit suggested by the theory, coincide and overlap each other. It is recalled that the flame propagates considerably faster at higher thermal expansion coefficients, see Fig. 4.1 (f). 
Figure 4.2 presents the flame acceleration rate versus the flame-related Reynolds number, with different expansion coefficients in each plot. The result resembles indirectly that of Fig.4.1. Indeed, it is clearly seen from Fig. 4.2 that the solid and dashed lines start deviating from each other as soon as $\Theta$ goes below 8, which yields a certain threshold thermal expansion for the intrinsic validity of the asymptotic (2.10).

In spite of a certain discrepancy between the results of (2.8) and (2.10) in Figs. 4.2 (e, f), our numerical simulations demonstrate that, for $6 \leq \Theta<8$, a flame front still may (or may not) accelerate exponentially depending on other parameters, such as Re. Namely, the exponential acceleration regime breaks as soon as Re exceeds some threshold value, $\operatorname{Re} 20$, as shown by the vertical dotted lines in Figs. 4.2 (e, f). As a matter of fact, this effect does not agree with the main conclusion of Chapter 2 that the asymptotic (2.10) still works more or less fine for $6 \leq \Theta<8$. Moreover (which is much more important!), while Chapter 2 and the study (Demirgok and Akkerman, 2013) yield that agreement between Eqs. (2.8) and (2.10) improves with the increase in Re, here the opposite tendency is observed. Without any guarantee that the following idea is correct, it is hypothesized that such a discrepancy is devoted to additional effects that might appear in wide tubes, say, the development of the hydrodynamic (DarrieusLandau; DL) instability that can modify a self-similar manner of the flame acceleration. 

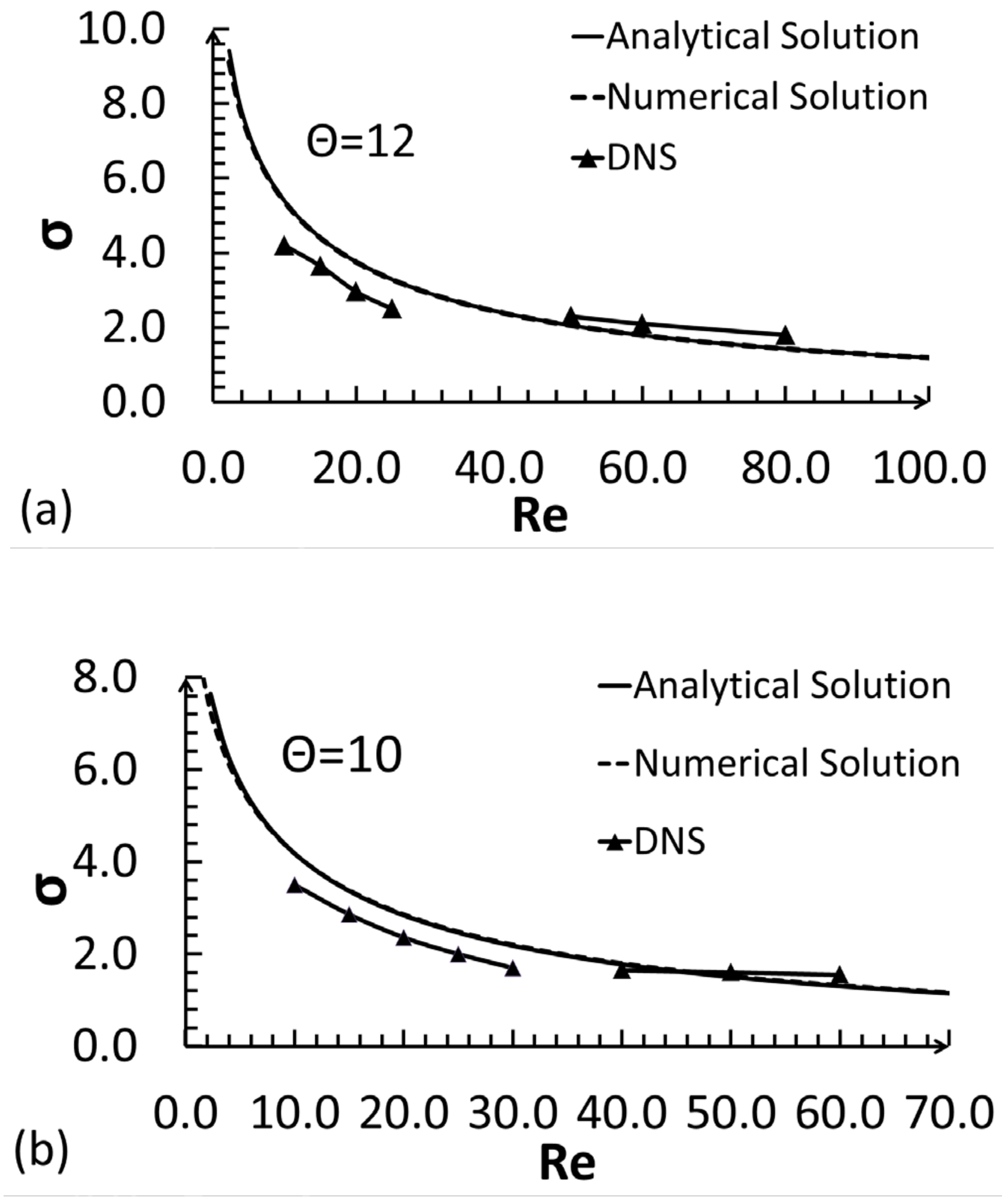

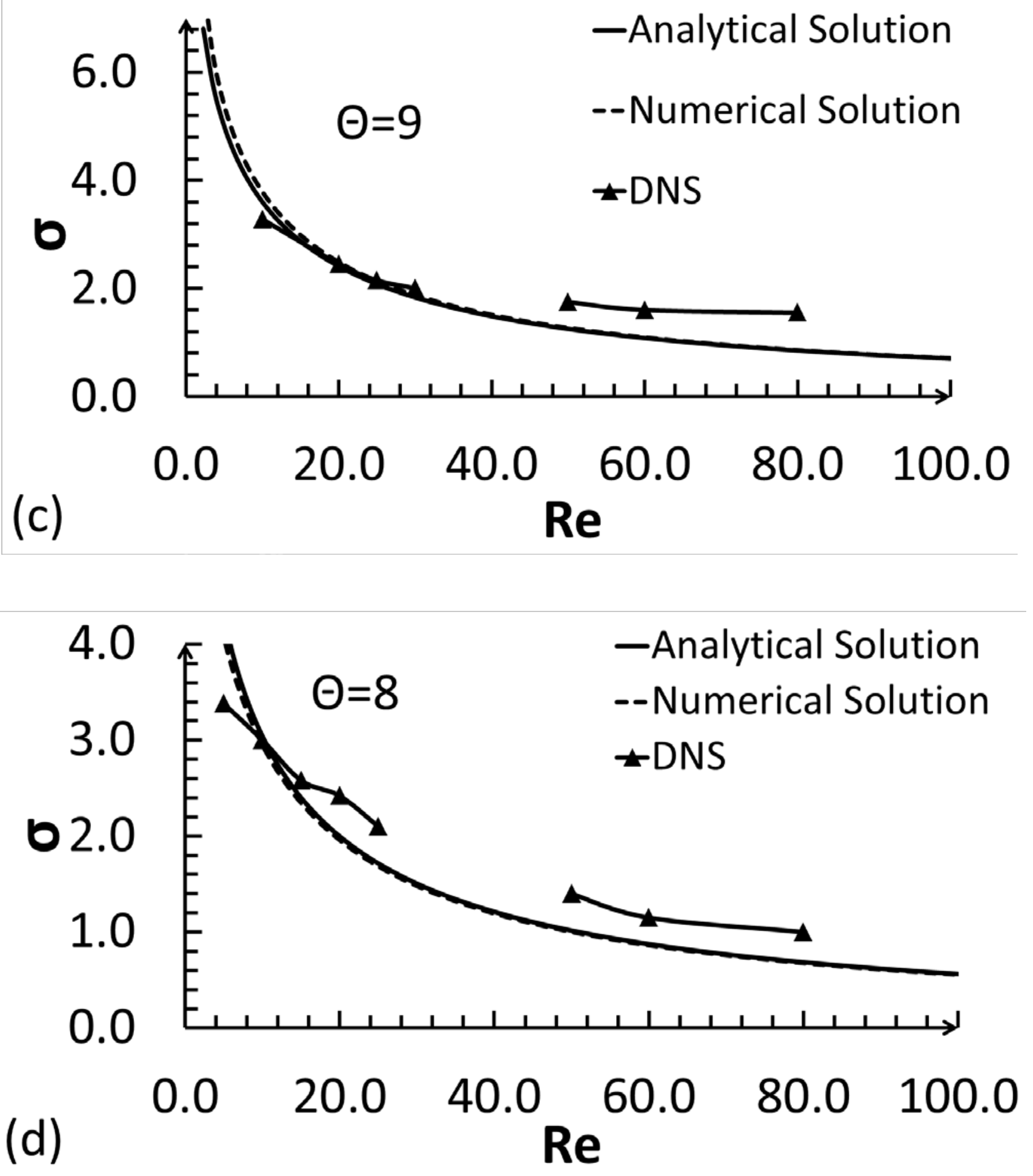

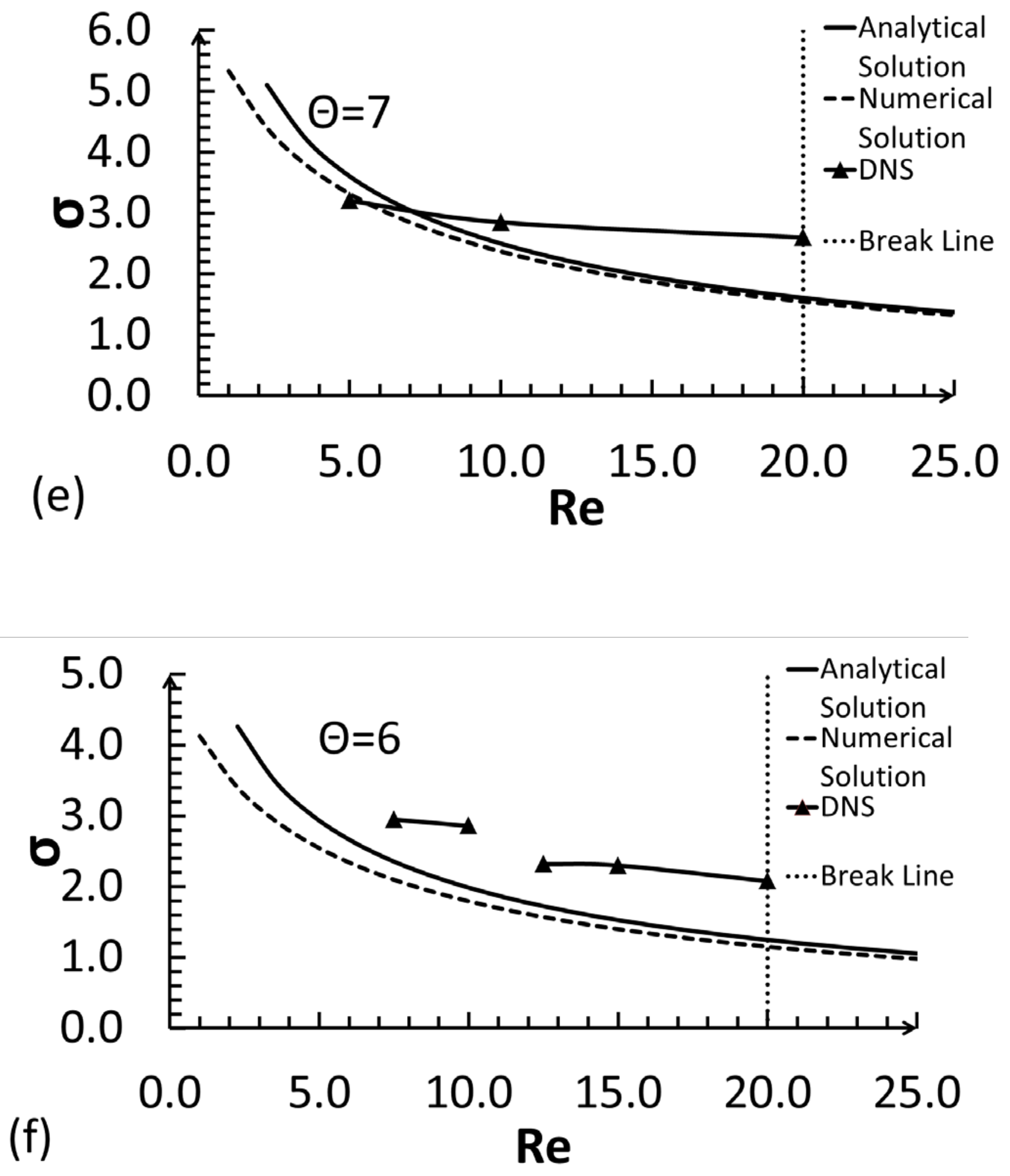

FIG. 4.2. The flame acceleration rate $\sigma$ versus propagation Reynolds number Re at thermal expansion $\Theta=$ 12 (a), 10 (b), 9 (c), 8 (d), 7 (e), 6 (f) 
It is noted that while one can observe excellent agreement between the results of (2.8), (2.10) and the DNS when $\Theta \geq 8$, such excellent agreement degrades as soon as $\Theta<8$ is taken. Indeed, agreement is quite limited for $6 \leq \Theta<8$, and one faces no agreement at all (no exponential trend observed) for $\Theta<6$.

Fig. 4.3a presents the evolution of the locus of the flame tip for the flame propagation Reynolds number $\operatorname{Re}=30$ and the expansion coefficient $\Theta=5$, obtained computationally. Figure 4.3b shows the scaled flame tip velocity $U_{\text {tip }} / U_{f}$ versus the scaled time $\tau=t U_{f} / R$ at the fixed thermal expansion coefficient $\Theta=5$ and the flame propagation Reynolds numbers $\mathrm{Re}=30$. Here one can see no agreement with the theory (Bychkov et al., 2005) at all: unlike the theoretical prediction, no exponential trend is observed in our simulations.

Similarly, the scaled flame tip velocity $\mathrm{U}_{\text {tip }} / \mathrm{U}_{\mathrm{f}}$ in Fig. 4.3b does not fit an exponential trend as well. Moreover, there are oscillations happening in the evolution of scaled flame velocity. Again, it is assumed that such a discrepancy is related to additional effects that might appear not only in wide tubes but also in a combustion process having a low thermal expansion that can somehow modify a self-similar manner of the flame acceleration.

Figures 4.3 (c, d) are the counterparts of Fig. 4.3 (a, b) for $\Theta=4$. They yield the same result: neither the evolution of the flame tip position, nor its speed exhibit the exponential accelerative dynamics. Anyway, as soon as $\Theta$ goes below 6, the exponential acceleration in the simulations is not observed at all, and this qualitatively fits both the original paper (Bychkov et al., 2005) and its justification (Demirgok and Akkerman, 2013). It is emphasized that the case of $\Theta=4$ conceptually differs from that of, say, $\Theta=9$. 
It is noted that only simulation results are demonstrated in Fig 4.3 (no theoretical ones). This is quite reasonable, because the evolution of the flame tip is not exponential anymore, and one certainly cannot compare this result with the theory based on the exponential approximation.
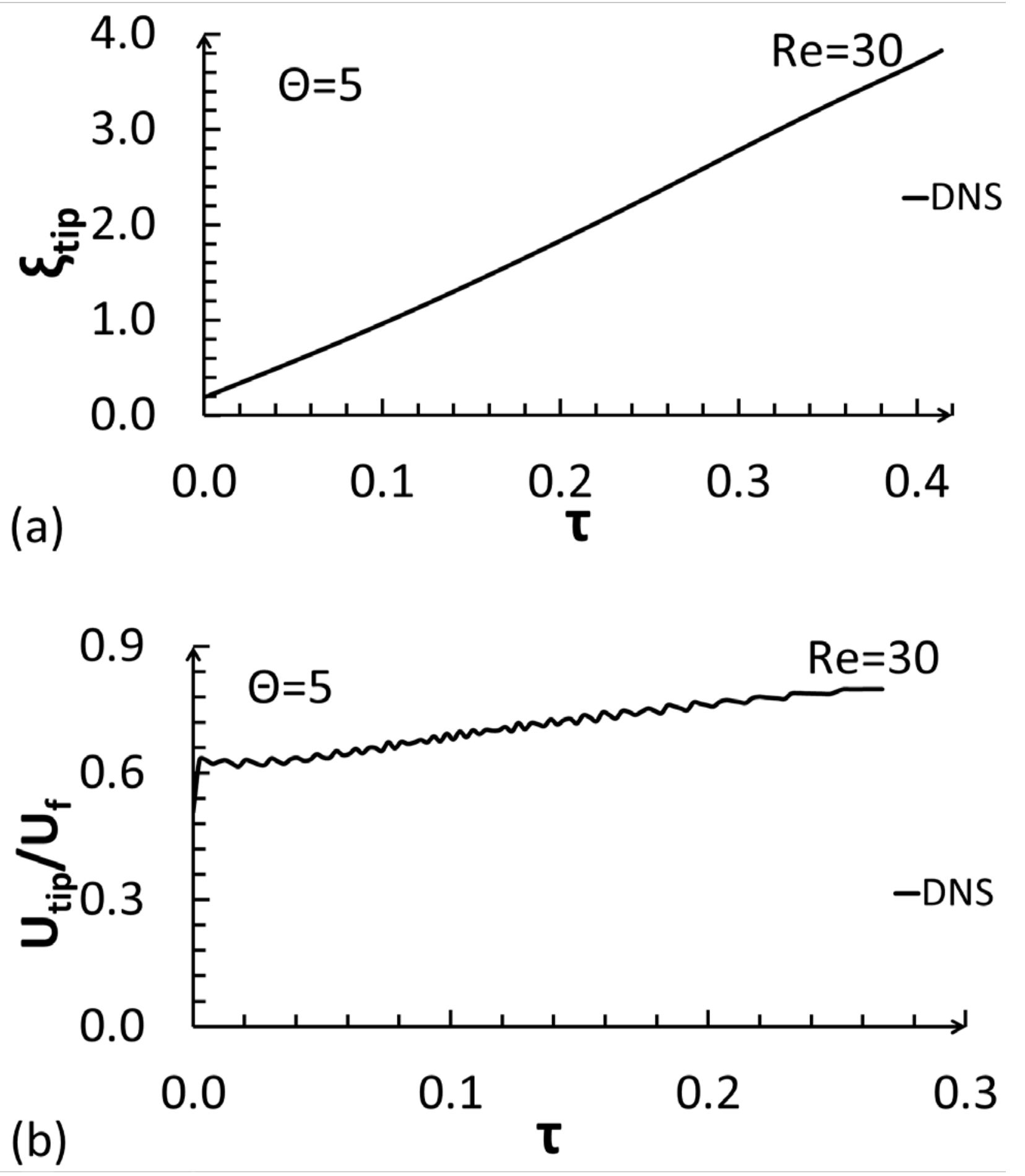

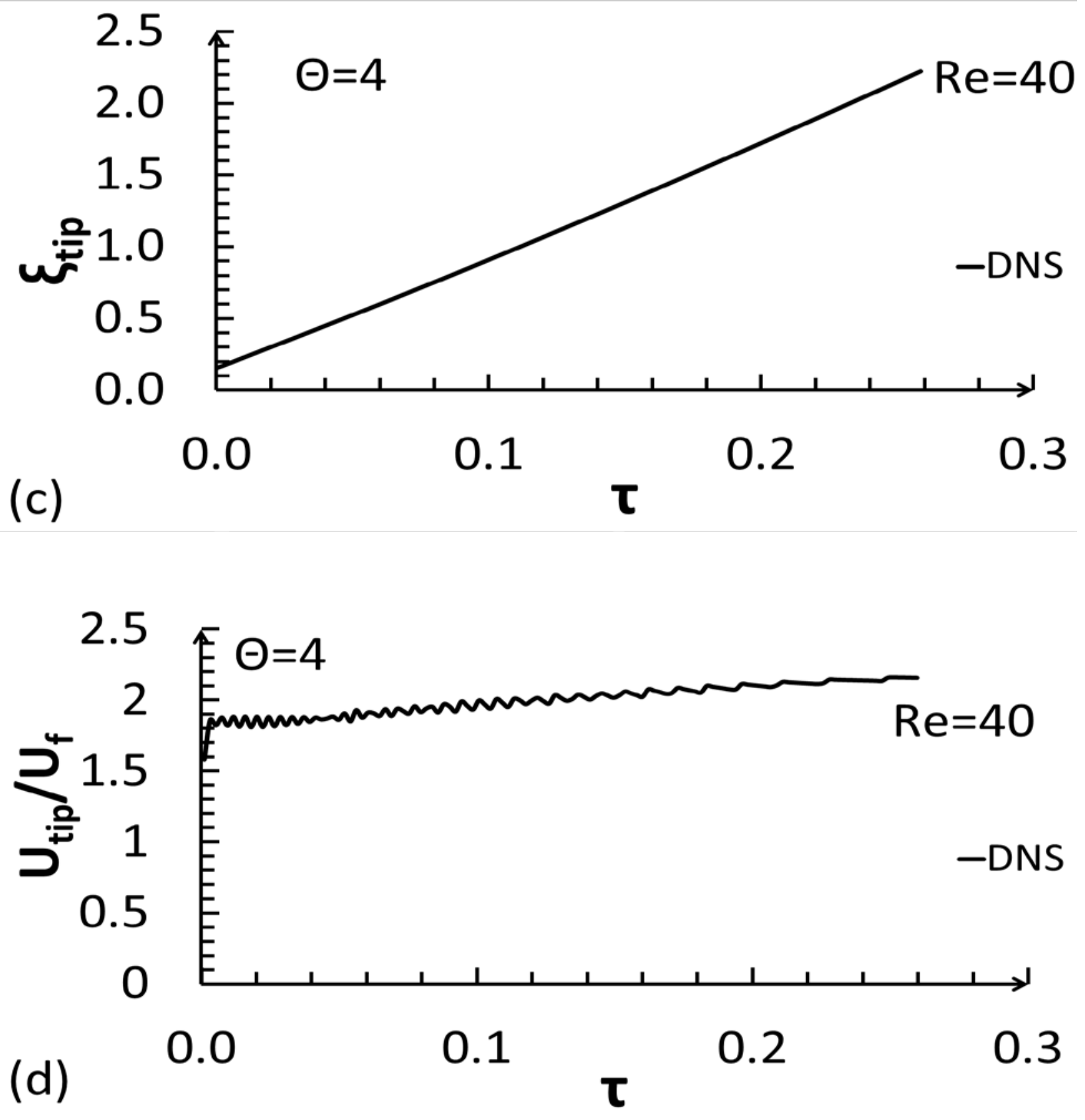

FIG. 4.3. The scaled flame tip position $\xi_{\text {tip }}$ versus the scaled time $\tau$ at fixed thermal expansion coefficient $\Theta=5$ and flame propagation Reynolds numbers $R e=30$ (a). The scaled flame tip velocity $U_{\text {tip }} / U_{f}$ versus the scaled time $\tau$ at fixed thermal expansion coefficient $\Theta=5$ and flame propagation Reynolds numbers $\operatorname{Re}=30$ (b). The scaled flame tip position $\xi_{\text {tip }}$ versus the scaled time $\tau$ at fixed thermal expansion coefficient $\Theta=4$ and flame propagation Reynolds numbers $R e=40$ (c). The scaled flame tip velocity $U_{\text {tip }}$ $/ \mathrm{U}_{\mathrm{f}}$ versus the scaled time $\tau$ at fixed thermal expansion coefficient $\Theta=4$ and flame propagation Reynolds numbers $\operatorname{Re}=40(\mathrm{~d})$ 
Finally, Fig. 4.4 presents the evolutions of the flame propagation in tubes in terms of color temperature snapshots. Specifically, Figs. 4.4 (a-c) are devoted to the flame of small thermal expansion, $\Theta=4$, while Figs. 4.4 (d-f) correspond to the realistically large one, $\Theta=9$. It is seen that the flame dynamics differ in these cases. In particular, a cave instead of a flame tip for $\Theta=4$ is observed while the flame front exhibits a convex, finger-like shape for $\Theta=9$. The reason for why one cannot observe finger-like shape for $\Theta=4$ is that the additional effect may appear in a situation where the thermal expansion factor is low, say, the development of the hydrodynamics (Darrieus-Landeu: DL) instability mentioned by Bychkov and Liberman, 2000 that can modify the flame tip evolution.
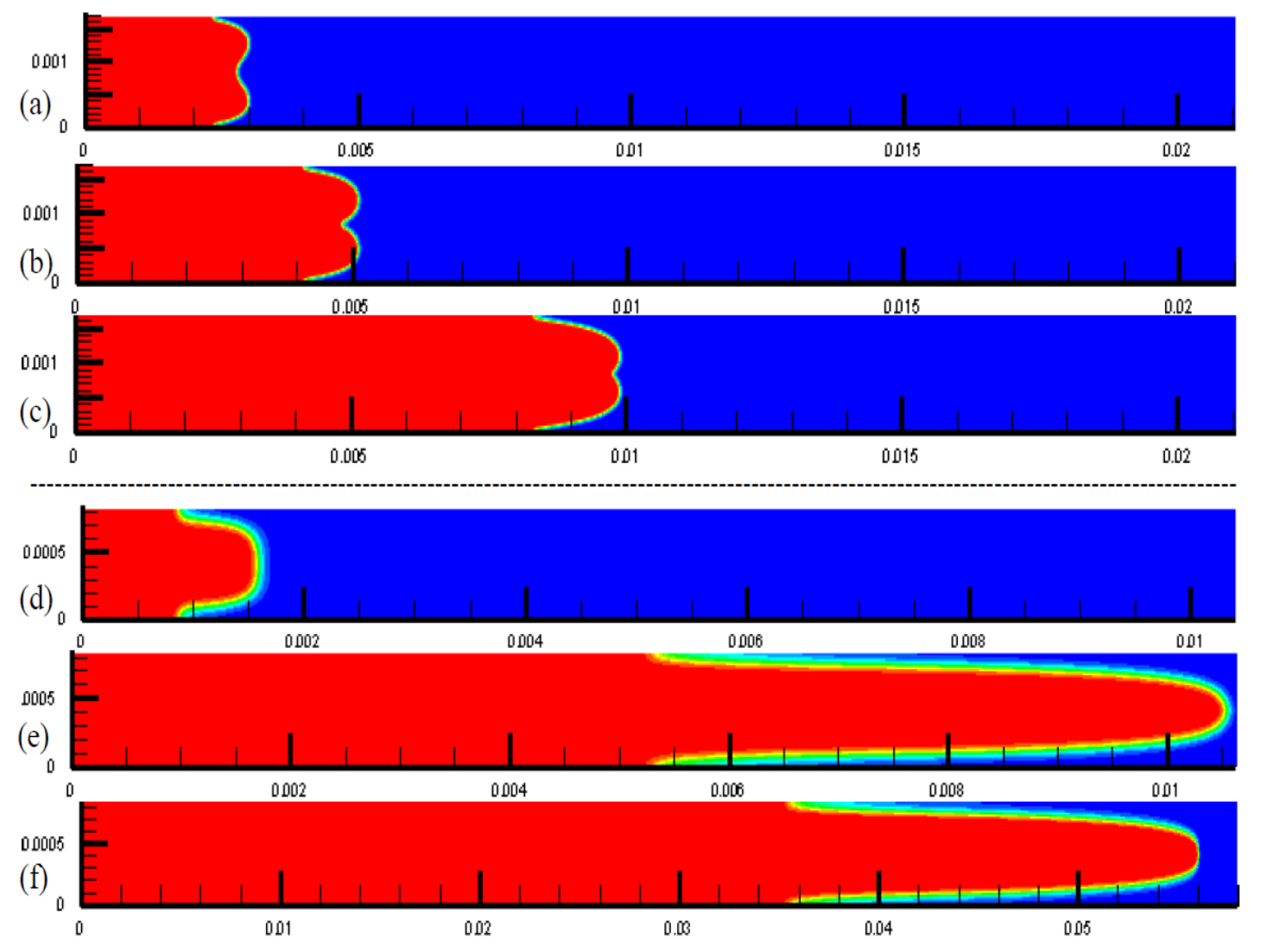

FIG. 4.4. Evolution of the flame shape for a small thermal expansion, $\Theta=4$ Fig. 4.3 (a-c) as well as for the realistically large one, $\Theta=9$ Fig. 4.3 (d-f) 


\subsection{Conclusions}

In this chapter, the analytical (Bychkov et al., 2005), semi-analytical, Chapter 2 and (Demirgok and Akkerman, 2013), and fully computational (DNS) approaches to flame acceleration in tubes due to wall friction are reconciled. It is shown that while the theory predicts the main characteristics of the flame acceleration very well when both $\Theta$ and Re are large enough, it may or may not be as accurate otherwise. In particular, it can be concluded that the theory quantitatively deteriorates for $6 \leq \Theta<8$, though it still may or may not work qualitatively (exhibiting the exponential acceleration or not), depending on Re. Finally, the theory breaks as soon as the expansion factor goes below 6 . 


\section{Chapter 5: Analysis of Ethylene-Oxygen Combustion in Micro-Pipes}

Propagation of stoichiometric ethylene-oxygen premixed flames in cylindrical pipes of sub/nearmillimeter radii is investigated computationally and analytically. Then computational and analytical results are compared to the recent experiments (Wu and Wang, 2011). Namely, various stages of flame evolution such as quasi-isobaric, exponential acceleration; its moderation due to gas compression; and eventual saturation to the Chapman-Jouget deflagration are detected. Specifically, the dynamics and morphology of the flame front, its propagation velocity and acceleration rate are determined. Due to viscous heating, the entire process can be followed by the detonation initiation ahead of the flame front. The computational component of this research is based on the computational platform described in Chapter 3. The overall study bridges the gap between the experiments (Wu and Wang, 2011) and the analytical formulation (Akkerman et al., 2006).

\subsection{Formulation}

Recent experiments have shown the occurrence of acceleration and DDT for ethylene-oxygen flames in micro-tubes/channels with diameter $\sim 1 \mathrm{~mm}$ (Wu and Wang, 2011). However, in contrast to the theories discussed in Chapter 2, which predict an exponential acceleration of laminar flames in micro-scale tubes at the initial stage, the experiments demonstrated a number of specific effects beyond the scope of the incompressible flow adopted in these analyses, such as moderated flame acceleration with subsequent saturation of the flame velocity to a steady value, which can be interpreted as the Chapman-Jouguet (CJ) deflagration speed.

To elucidate these observations, in particular, the experiments in micro-tubes, the role of gas compression has been investigated in the acceleration process. Specifically, the incompressible 
theory of flame acceleration (Akkerman et al., 2006) has been extend to include small but finite gas compression, by employing expansion in the initial Mach number up to $1^{\text {st }}$ order terms. The analysis shows how compression waves modify the regime of flame acceleration, the flame shape and velocity as well as the upstream flow. Analytical expressions for the main characteristics of the flame dynamics are derived. Specifically, the average flame front velocity in the laboratory frame and the average flame position read

$$
\dot{\Sigma}=\frac{\Theta \Omega_{s s} \exp (\sigma \tau)}{1+C_{1} \Theta \Omega_{s s} \exp (\sigma \tau)}, \quad \quad \Sigma=\frac{1}{\sigma C_{2}} \ln \left[1+C_{2} \Theta \Omega_{s s} \exp (\sigma \tau)\right]
$$

where the flame tip parameters (acceleration, velocity and position) are given by

$$
\begin{aligned}
& \ddot{\xi}_{\text {tip }}=\sigma \dot{\xi}_{\text {tip }}\left(1-C_{2} \dot{\xi}_{\text {tip }}\right), \\
& \dot{\xi}_{\text {tip }}=\frac{(\Theta+\sigma / \mu) \Omega_{s s} \exp (\sigma \tau)}{1+C_{2}(\Theta+\sigma / \mu) \Omega_{s s} \exp (\sigma \tau)}, \\
& \xi_{\text {tip }}=\frac{1}{\sigma C_{2}} \ln \left[1+C_{2}(\Theta+\sigma / \mu) \Omega_{\text {ss }} \exp (\sigma \tau)\right] .
\end{aligned}
$$

Here $\Omega_{s s}$ stands for the scaled flame velocity by the instant when a self-similar manner of the flame acceleration is attained; $\sigma$ is given by Eq. (2.16) in the $0^{\text {th }}$ order approximation of $\mu^{-1}<<1$, and Eq. (2.17) in the $1^{\text {st }}$ order approximation, with $\mu \equiv \sqrt{\sigma \mathrm{Re}}$ in both cases; and the following designations are introduced:

$$
\begin{aligned}
& C_{1}=\frac{U_{f}}{c_{s}}\left(\frac{\Theta-1}{\Theta}\right)^{2} A(1+B), \\
& C_{2}=\frac{U_{f}}{c_{s}} \frac{(\Theta-1) A}{(\Theta+\sigma / \mu)^{2}}\left(\frac{1+B}{2 \sigma}+\frac{B}{\sqrt{2} \mu}\right) \approx A \frac{U_{f}}{C_{s}}\left(\frac{\Theta-1}{\Theta}\right)^{2}, \\
& B=\frac{1}{\sigma+(\mu / \sqrt{2})}, \quad A=[(\gamma-1)(\Theta-\tilde{m})+\Theta]\left(\frac{1}{\Theta-1}-B\right),
\end{aligned}
$$


where $c_{s}$ is the sound speed in the fresh gas and $\tilde{m}$ the ratio of the average molecular weights of unburned and burned gases, $\tilde{m}=m_{f} / m_{b}$. The details of the derivations are presented in Appendix C.

\subsection{Experiments}

This section briefly describes the experiments (Wu and Wang, 2011). The effects of the characteristic length scale and the premixture equivalence ratio on the ethylene-oxygen flame propagation in capillary tubes were experimentally analyzed using high speed cinematography. The inner radii of tubes investigated were $0.25,0.5,1$ and $1.5 \mathrm{~mm}$. The flame was ignited at the center of the $1.5 \mathrm{~m}$ long smooth tube under ambient pressure and temperature before propagated towards the exits in the opposite directions.

A total of five reaction propagation scenarios, including DDT transition followed by steady detonation wave transmission, oscillating flame, steady deflagration, galloping detonation and quenching flame, were identified. Briefly, transparent borosilicate capillary tubes open at both ends were used as the flame propagation channels. Reaction wave propagations are visualized through the transparent tub using a high speed camera (IDT, Motionscope X3). Since ignition is achieved by applying high voltage across the electrodes at the center of the $1.5 \mathrm{~m}$ long tubes, the equivalent tube length for reaction propagation is therefore $0.75 \mathrm{~m}$. The ethylene-oxygen mixture was premixed using a tee before being fed into a tube. The flow rates of the oxygen and the fuel were controlled using the mass flow controllers (MKS 1179A). The reader can see the original paper (Wu and Wang, 2011) for further details. 


\subsection{Computational Simulations}

Extensive direct numerical simulations of the hydrodynamic and combustion equations including Arrhenius chemical kinetics and transport processes have been performed in an axisymmetric, cylindrical geometry, the basic equations read

$$
\begin{aligned}
& \frac{\partial}{\partial t} \rho+\frac{1}{r} \frac{\partial}{\partial r}\left(r \rho u_{r}\right)+\frac{\partial}{\partial z}\left(\rho u_{z}\right)=0, \quad \frac{\partial}{\partial t}\left(\rho u_{r}\right)+(\mathbf{u} \cdot \nabla)\left(\rho u_{r}\right)-\rho \frac{u_{\theta}^{2}}{r}=-\frac{\partial P}{\partial r}+\zeta\left(\nabla^{2} u_{r}-\frac{u_{r}}{r^{2}}\right), \\
& \frac{\partial}{\partial t}\left(\rho u_{r}\right)+(\mathbf{u} \cdot \nabla)\left(\rho u_{\theta}\right)+\rho \frac{u_{\theta} u_{r}}{r}=-\frac{\partial P}{\partial r}+\zeta\left(\nabla^{2} u_{\theta}-\frac{u_{\theta}}{r^{2}}\right), \\
& \frac{\partial}{\partial t}\left(\rho u_{z}\right)+(\mathbf{u} \cdot \nabla)\left(\rho u_{z}\right)=-\frac{\partial P}{\partial z}+\zeta \nabla^{2} u_{z} \\
& \frac{\partial}{\partial t}\left(\rho \varepsilon+\frac{1}{2} \rho \mathbf{u}^{2}\right)+\frac{1}{r} \frac{\partial}{\partial r}\left(\rho r u_{r} h+\frac{r}{2} \rho u_{r} \mathbf{u}^{2}+r q_{r}-r u_{r} \gamma_{r, r}-r u_{z} \gamma_{r, z}-r u_{\theta} \gamma_{r, \theta}\right) \\
& \quad+\frac{\partial}{\partial z}\left(\rho u_{z} h+\frac{1}{2} \rho u_{z} \mathbf{u}^{2}+q_{z}-u_{z} \gamma_{z, z}-u_{r} \gamma_{r, z}-u_{\theta} \gamma_{z, \theta}\right)=0, \\
& \frac{\partial}{\partial t}(\rho Y)+\frac{1}{r} \frac{\partial}{\partial r}\left(r \rho u_{r} Y-r \frac{\zeta}{S c} \frac{\partial Y}{\partial r}\right)+\frac{\partial}{\partial z}\left(\rho u_{z} Y-\frac{\zeta}{S c} \frac{\partial Y}{\partial z}\right)=-\frac{\rho Y}{\tau_{R}} \exp \left(-E_{a} / R_{p} T\right), \\
& (\mathbf{u} \cdot \nabla) \equiv u_{r} \frac{\partial}{\partial r}+u_{z} \frac{\partial}{\partial z}, \quad, \nabla^{2} \equiv \frac{1}{r} \frac{\partial}{\partial r}\left(r \frac{\partial}{\partial r}\right)+\frac{\partial^{2}}{\partial z^{2}},
\end{aligned}
$$

the stress tensor $\gamma_{i, j}$ takes the form

$$
\gamma_{r, r}=2 \zeta \frac{\partial u_{r}}{\partial r}, \quad \gamma_{r, \theta}=\zeta\left(\frac{\partial u_{\theta}}{\partial r}-\frac{u_{\theta}}{r}\right), \quad \gamma_{r, z}=\zeta\left(\frac{\partial u_{z}}{\partial r}+\frac{\partial u_{r}}{\partial z}\right), \quad \gamma_{z, z}=2 \zeta \frac{\partial u_{z}}{\partial z}, \quad \gamma_{z, z}=2 \zeta \frac{\partial u_{z}}{\partial z},
$$

and the energy diffusion vector $q_{i}$ is

$$
q_{r}=-\zeta\left(\frac{C_{P}}{\operatorname{Pr}} \frac{\partial T}{\partial r}+\frac{Q}{\mathrm{Sc}} \frac{\partial Y}{\partial r}\right), \quad q_{z}=-\zeta\left(\frac{C_{P}}{\operatorname{Pr}} \frac{\partial T}{\partial z}+\frac{Q}{\mathrm{Sc}} \frac{\partial Y}{\partial z}\right)
$$




\subsection{Results and Discussion}

In this section, new computational results are compared to the extension of the theory (Akkerman et al., 2006) and the experimental data on premixed stoichiometric ethylene-oxygen flames accelerating in tubes of small radii, $\mathrm{R}=0.25$ and $0.5 \mathrm{~mm}$. Following the GasEQ and USC Mech II results for stoichiometric ethylene-oxygen combustion, the kinematic viscosity $v=1.35 \times 10^{-5} \mathrm{~m}^{2} / \mathrm{s}$ and the laminar flame speed $U_{f}=3.58 \mathrm{~m} / \mathrm{s}$ are used, thereby making the flame propagation Reynolds number for such tubes to be about $\operatorname{Re}=56 \sim 460$. The adiabatic exponent is $\gamma=c_{P} / c_{V}=1.34$ yielding the sound speed being $c_{s}=\sqrt{\gamma P / \rho_{0}}=331 \mathrm{~m} / \mathrm{s}$, with the initial Mach number $M_{0}=U_{f} / c_{0}=0.0125$. With the initial expansion ratio of $\Theta=10.6$, one finds $\tilde{m}=\Theta /\left(T_{b 0} / T_{0}\right)=1.34$.

It is recalled that the evolution of the flame velocity in the experiments showed humps and cusps in the early stage of combustion, until a self-sustained state of flame acceleration is achieved. For a meaningful comparison, one therefore had to employ the initial experimental marker related to self-sustained flame acceleration as the initial condition for the theoretical formulation, with the experimental flame speed at that instant assuming the role of $\Omega_{s s}$, and hence considerably increasing the Mach number as compared to the initial one. Consequently, while the comparison becomes feasible for narrow tubes, $R=(0.25-0.5) \mathrm{mm}$, the self-sustained flame propagation in a wider tube, $R=(1.0-1.5) \mathrm{mm}$, appears outside the validity domain of the theory that assumes weak compressibility. 

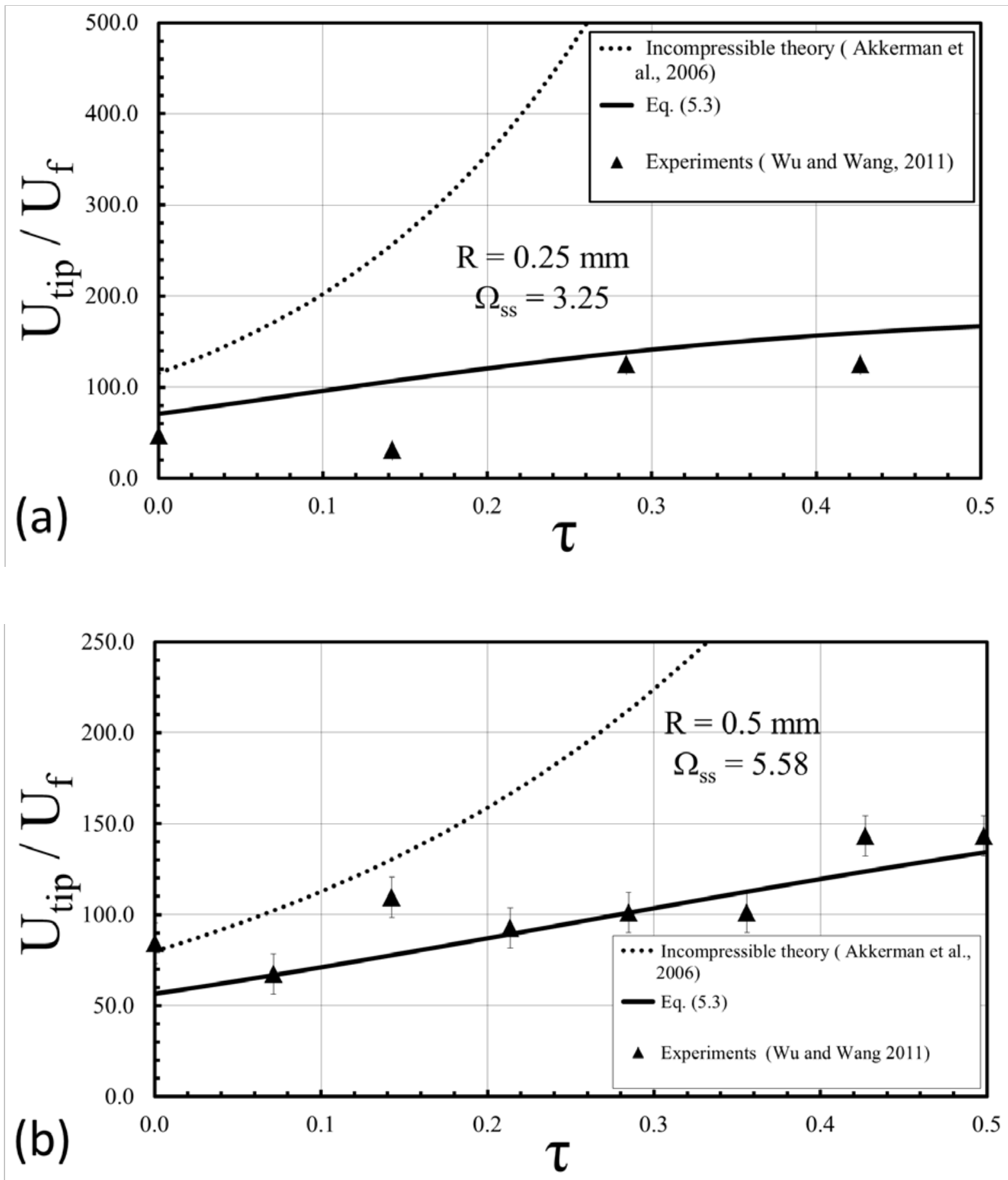

FIG. 5.1. Evolution of the flame tip in a tube of radii $\mathrm{R}=0.25 \mathrm{~mm}$ (a) and $0.5 \mathrm{~mm}$ (b) 


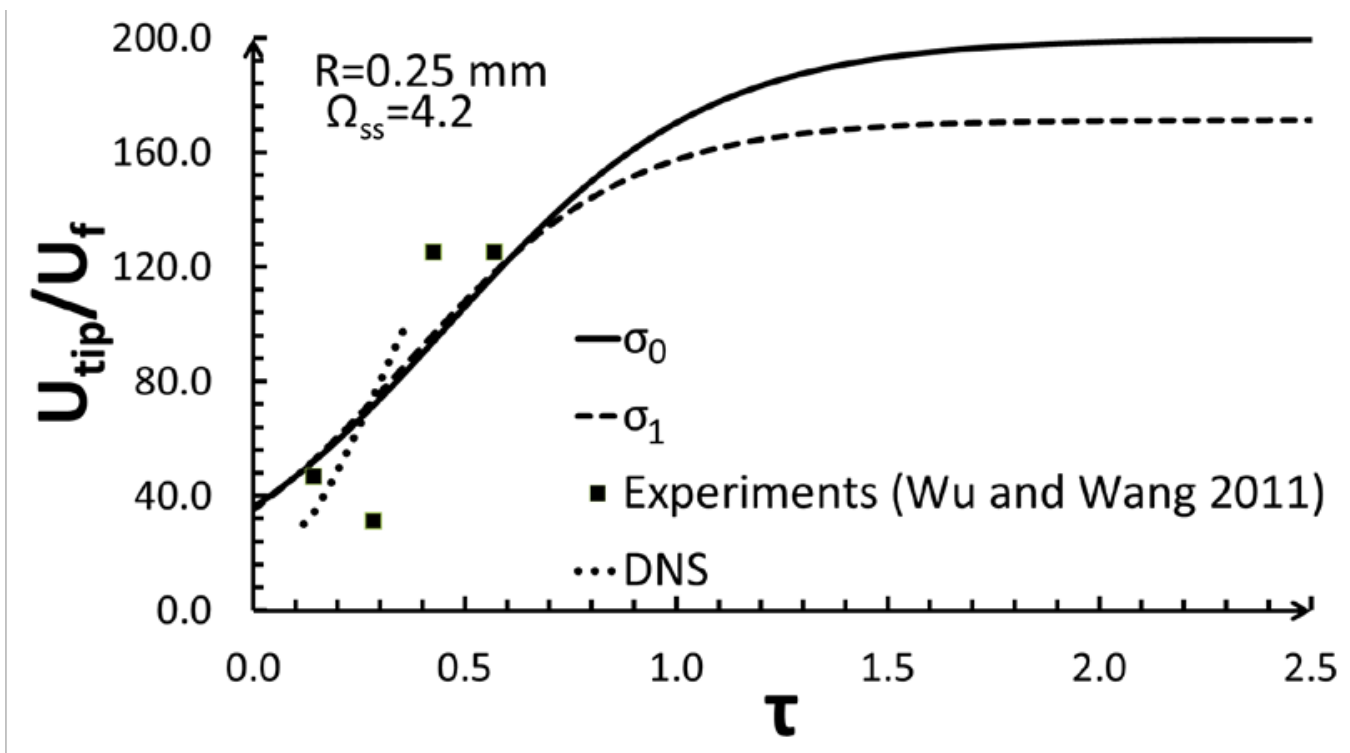

FIG. 5.2. Evolution of the flame tip in a tube of $\mathrm{R}=0.25 \mathrm{~mm}$ for $\Omega_{\mathrm{ss}}=4.2$. The cases of the acceleration rate given by Eq. (2.17), $\sigma_{1}$ and Eq. (2.16), $\sigma_{0}$, are shown by the dashed and solid lines, respectively. Filled rectangles show the experimental results while dotted line represents the DNS ones

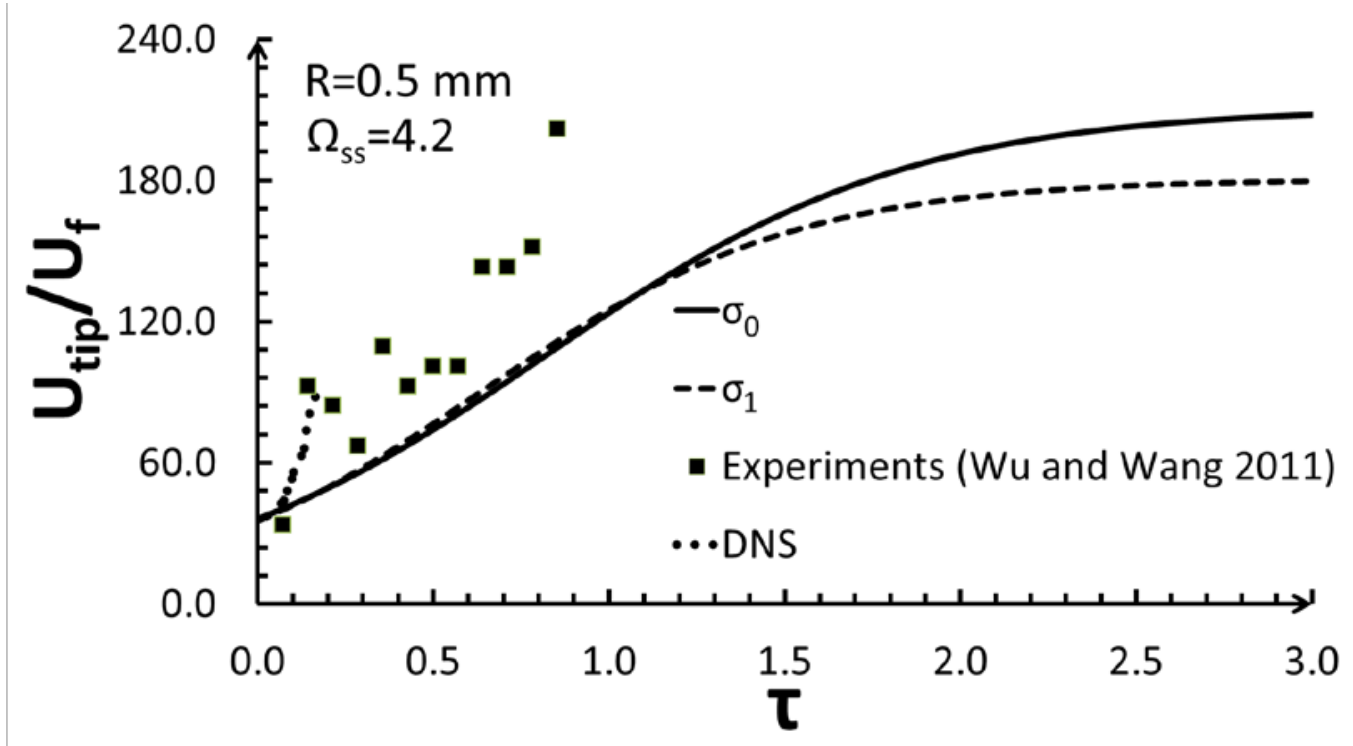

FIG. 5.3. Evolution of the flame tip in a tube of $\mathrm{R}=0.5 \mathrm{~mm}$ for $\Omega_{\mathrm{ss}}=4.2$. The cases of the acceleration rate given by Eq. (2.17), $\sigma_{1}$, and Eq. (2.16), $\sigma_{0}$, are shown by the dashed and solid lines, respectively. Filled rectangles show the experimental results while dotted line represents the DNS ones 


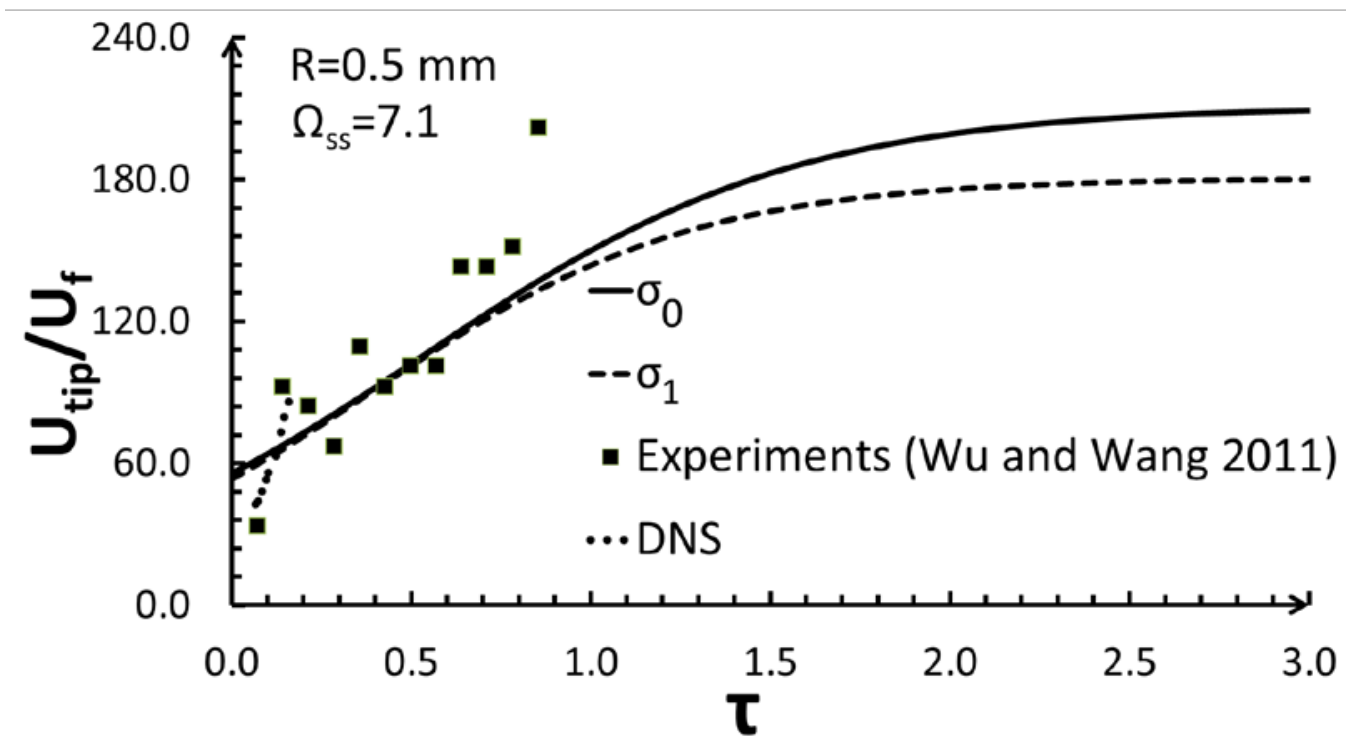

FIG. 5.4. Evolution of the flame tip in a tube of $\mathrm{R}=0.5 \mathrm{~mm}$ for $\Omega_{\mathrm{ss}}=7.1$. The cases of the acceleration rate given by Eq. (2.16), $\sigma_{1}$, and Eq. (5.16), $\sigma_{0}$, are shown by the dashed and solid lines, respectively. Filled rectangles show the experimental results while dotted line represents the DNS ones

Figures 5.1 - 5.4 compare the present computational simulations, theory and experiments for cylindrical tubes of radii $R=(0.25-0.5) \mathrm{mm}$. Specifically, the analytical and experimental results are compared in Fig. 5.1. Equation (5.3) is shown by the solid lines, while the dotted line is related to the incompressible theory (Akkerman et al., 2006). The experimental data are shown by markers.

The present computational results are compared to the analytical and experimental evolutions of the flame tip in Figs. 5.2 - 5.4. Specifically, Fig. 5.2 shows the case of $\mathrm{R}=0.25 \mathrm{~mm}$ with $\Omega_{\mathrm{ss}}=$ 4.2; while the other two plots are devoted to the twice wide tube: the situation of $\mathrm{R}=0.5 \mathrm{~mm}$ with $\Omega_{\mathrm{ss}}=4.2$ is shown in Fig. 5.3, while Fig. 5.4 presents that of $\mathrm{R}=0.5 \mathrm{~mm}$ with $\Omega_{\mathrm{ss}}=7.1$. In all of these three figures, $5.2-5.4$, the solid lines represent the $0^{\text {th }}$ order approach, while the dashed lines show the $1^{\text {st }}$ order approach. Filled rectangles are devoted to the experimental 
measurements, while present DNS results are shown by the dotted lines. Again, good agreement between the simulations, theoretical formulation and experiments is observed at the initial stage.

Finally, Fig. 5.5 presents the color temperature snapshots for the stoichiometric ethylene-oxygen combustion in a tube of radius $0.25 \mathrm{~mm}$, with the fuel mixture and burnt matter shown conventionally by the blue and red colors, respectively. The flame front exhibits a convex, finger-like shape.

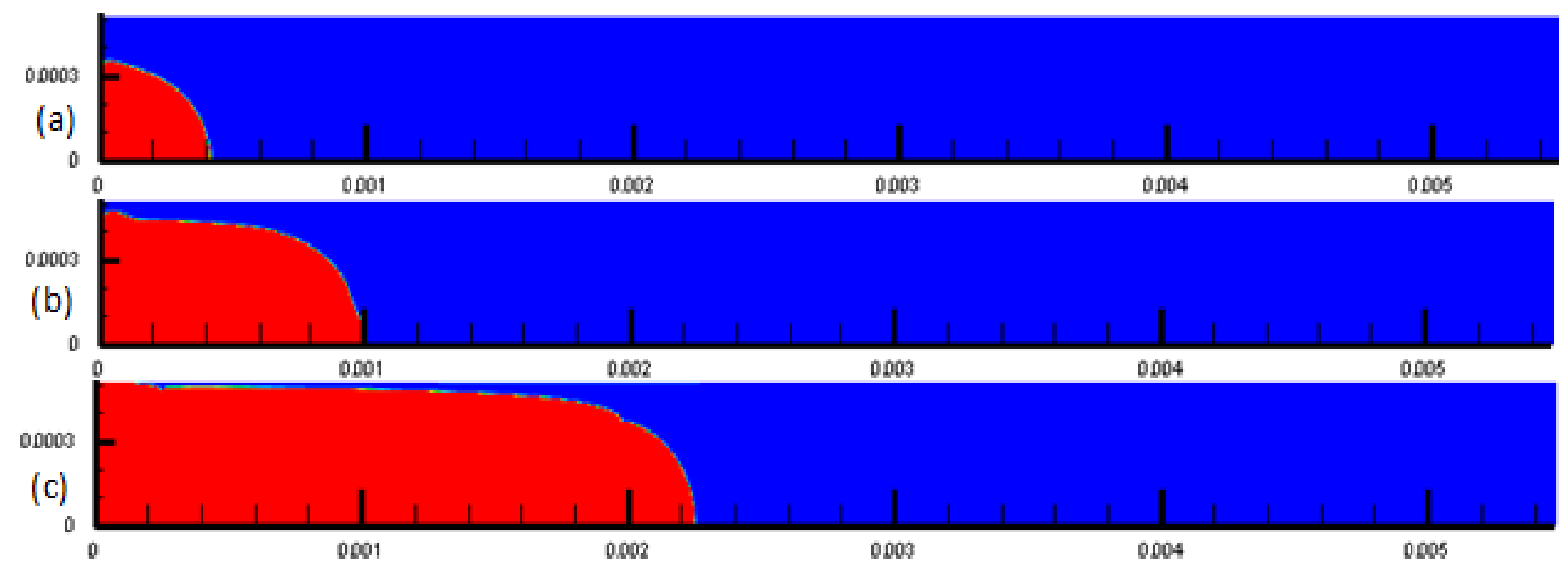

FIG. 5.5. Evolution of the flame shape in a tube of radius $R=56 \mathrm{~L}_{\mathrm{f}}=0.25 \mathrm{~mm}$. The colors represent the temperature from $300 \mathrm{~K}$ in the fuel mixture (blue) to the corresponding burnt matter temperature (red)

\subsection{Conclusion}

Dynamics and morphology of stoichiometric ethylene-oxygen flames in sub-millimeter cylindrical pipes is studied computationally, analytically and experimentally. The main flame characteristics such as the flame shape and velocity, locus and speed of the flame tip, the flame acceleration rate, as well as the flow field generated by the flame propagation were analyzed. Good agreement between all approaches employed at the initial stage is demonstrated which thereby validates the analytical formulation on flame acceleration in tubes. 


\section{Chapter 6: Summary}

The present thesis had several objectives devoted to the comprehensive (analytical, computational and experimental) investigation of the flame acceleration in tubes/channels due to wall friction. A "road map" for this work was the theoretical formulation of Bychkov and coauthors who have revealed the entire scenario of flame acceleration and detonation triggering. One of the objectives of this thesis was to investigate the intrinsic limitations and accuracy of the theories (Bychkov et al., 2005; Akkerman et al., 2006). This task was performed by utilizing the Newton-Raphson and numerical discretization methods. The limitations and accuracy of the theories were clearly underlined in the Re- $\Theta$ diagram. Another objective of the thesis was to identify the influence of the thermal expansion and on the intrinsic accuracy of the theory as well as on the flame propagation in general. It was clearly shown that the theory predicts the main characteristics of the flame acceleration very well when both $\Theta$ and Re are large enough. However, the theory quantitatively deteriorates for $6 \leq \Theta<8$ depending on Re. Eventually, the theory breaks as soon as the expansion factor goes below 6. Finally, the last objective was to scrutinize the dynamics and morphology of stoichiometric ethylene-oxygen flames in submillimeter cylindrical pipes and bridge the gap between the experiments done by $\mathrm{Wu}$ and $\mathrm{Wang}$ (2011) and our analytical formulation by using DNS. In this part, the main flame characteristics such as the flame shape and velocity, the locus and speed of the flame tip, the acceleration rate, as well as the flow field generated by the flame propagation are determined, and good agreement between all approaches (analytical, computational and experimental) is found at the initially state of the flame acceleration. This thereby validates the recent analytical formulations (Bychkov et al., 2006; Akkerman et al., 2006; Bychkov et al., 2010). 


\section{References}

V. Akkerman, V. Bychkov, A. Petchenko, L.-E. Eriksson, Combust. Flame 145 206-219 (2006).

V. Akkerman, V. Bychkov, and L. E. Eriksson, Combust.Flame 151, 452 (2007).

V. Akkerman, V. Bychkov, L. de Goey, R. Bastiaans, J. van Oijen, and L.-E. Eriksson, Phys.

Fluids 20, 055107 (2008).

V. Akkerman, C.K. Law and V. Bychkov, Phys. Rev. E 83, 026305 (2011).

V.Akkerman, C.K Law, V. Bychkov, L.E. Eriksson, Phys. Fluids 22, 053606 (2010).

N. Anderson, Eriksson, L.-E., and Davidson, L., AIAA Journal 43, p. 1899 (2005).

V. Bychkov, A. Petchenko, V. Akkerman, L.E. Eriksson, Phys. Rev. E 72, 046307 (2005).

V. Bychkov, V. Akkerman, Phys. Rev. E 73, 066305 (2006).

V. Bychkov, M.A Liberman, Physical Reorts 325, 115-237 (2000).

V. Bychkov, D. Valiev, L.-E. Eriksson,. 101164501 (2008).

V. Bychkov, V. Akkerman, G. Fru, A. Petchenko, L.E, Eriksson, Combust. Flame 150, 263 (2007).

V. Bychkov, V. Akkerman, D. Valiev, C.K. Law, Phys. Rev. E 81, 026309 (2010).

V. Bychkov, A. Petchenko, V. Akkerman, and L.E. Eriksson, Phys. Rev. E 72, 046307 (2005).

B. Demirgok, V. Akkerman, Theory of Flame Acceleration in Tubes due to Wall Friction:

Intrinsic Limitations \& Accuracy. $8^{\text {th }}$ U. S. Nat. Combust. Meeting, Canyon, UT, May 19-22 (2013).

L.-E. Erikkson., Development and validation of highly modular flow solver versions in g2dflow and g3dflow series for compressible viscous reacting flow. VAC Report: p. 9970-1162. (1995).

L.-E. Erikkson., Comp. Meth. Appl. Mech. Eng. 64, 95 (1987).

G. Roy, S. Frolov, A. Borisov, and D. Netzer, Prog. En. Combust. Sci. 30, 545 (2004). 
K.I. Shelkin, Zh. Exp. Teor. Fiz. 10, 823 (1940).

J.E. Shepherd and J.H.S Lee, in Major Research Topics in Combustion, 439 (SpringerVerlag, Hampton, VA) (1992).

O. Travnikov, V. Bychkov, and M. Liberman, Phys. Rev. E 61, 468 (2002).

D. Valiev, V. Akkerman, M. Kuznetsov, L.E. Eriksson, C.K. Law, V. Bychkov, Combust. Flame 160, 97 (2013).

D. Valiev, V. Bychkov, V. Akkerman, L.-E. Eriksson, M. Marklund, Phys. Lett. A 372 27-28 4850-4857 (2008).

D. Valiev, V. Bychkov, V. Akkerman, L.-E. Eriksson, Phys. Rev. E 80, 036317 (2009).

D. Valiev, V. Bychkov, V. Akkerman et al., Combust. Flame 157, 1012 (2010).

C. Wollblad, L. Davidson, L.-E. Eriksson, AIAA J. 44, 2340 (2006).

M.-H. Wu, M. Burke, S. Son, R. Yetter, Proc. Combust. Inst. 31 2429-2436 (2007).

M.-H. Wu, C.-Y. Wang, Proc. Combust. Inst. 33 2287-2293 (2011). 


\section{APPENDIX: A}

“Semi-analytical” Matlab code developed for the 2D theory.

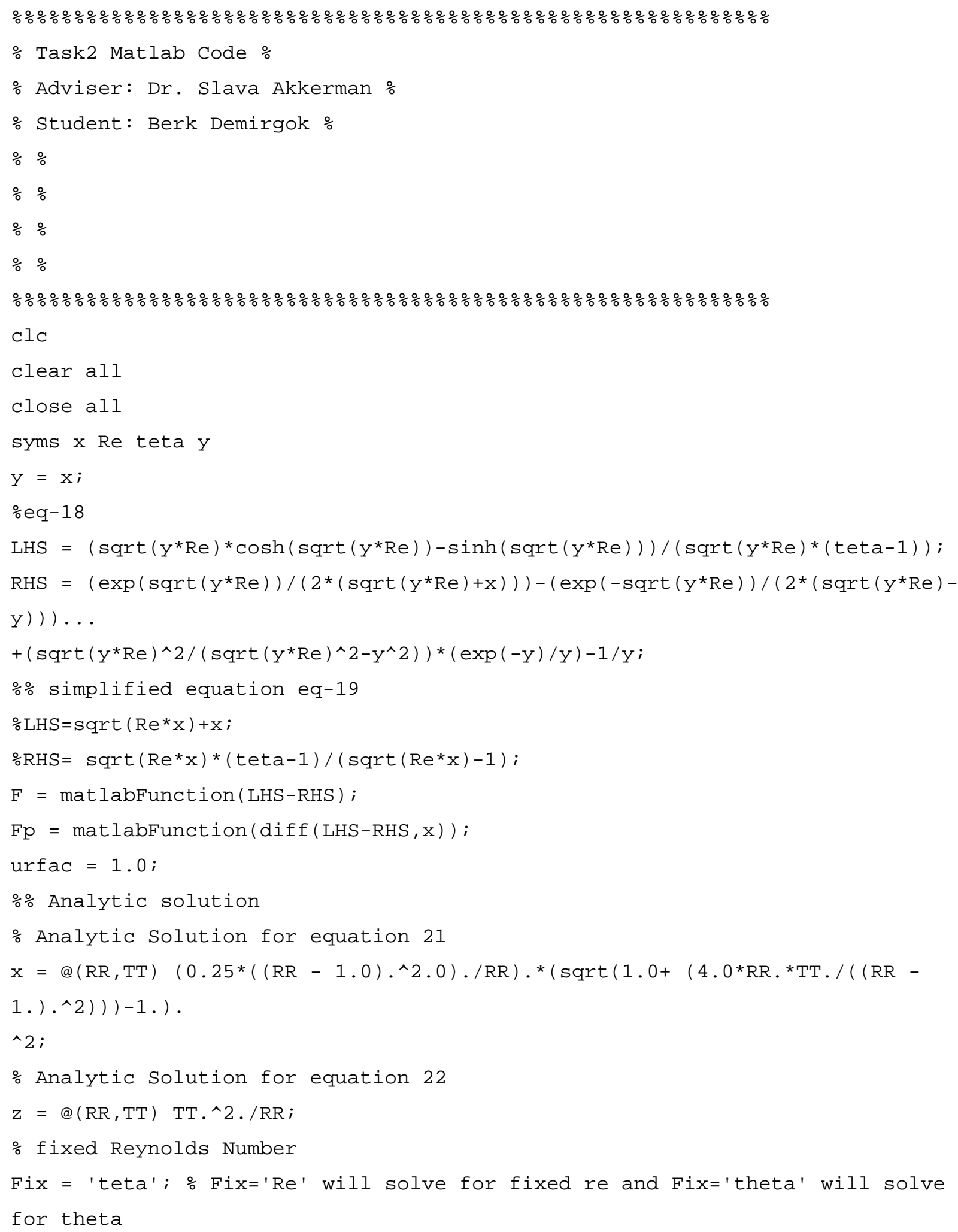




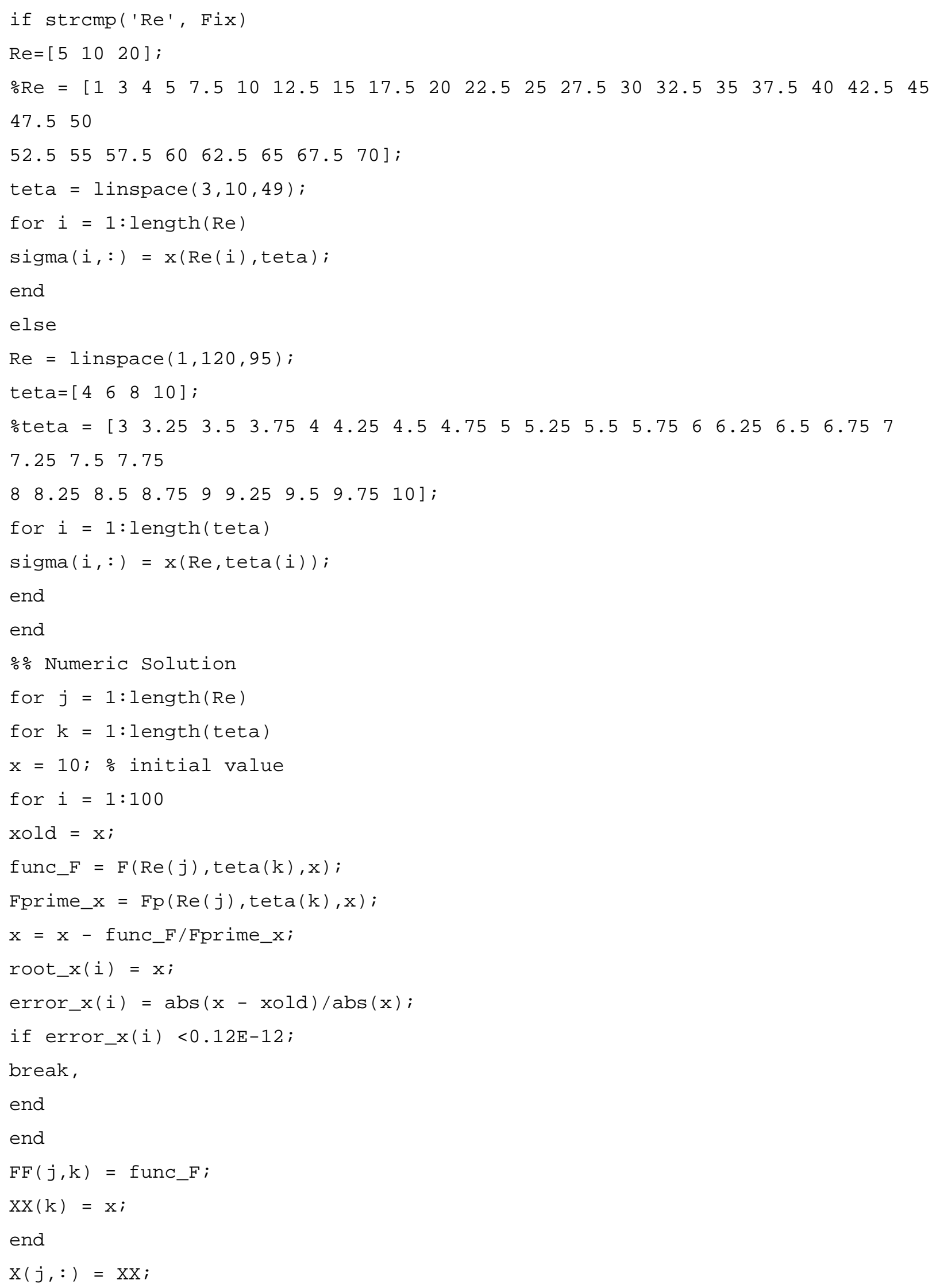




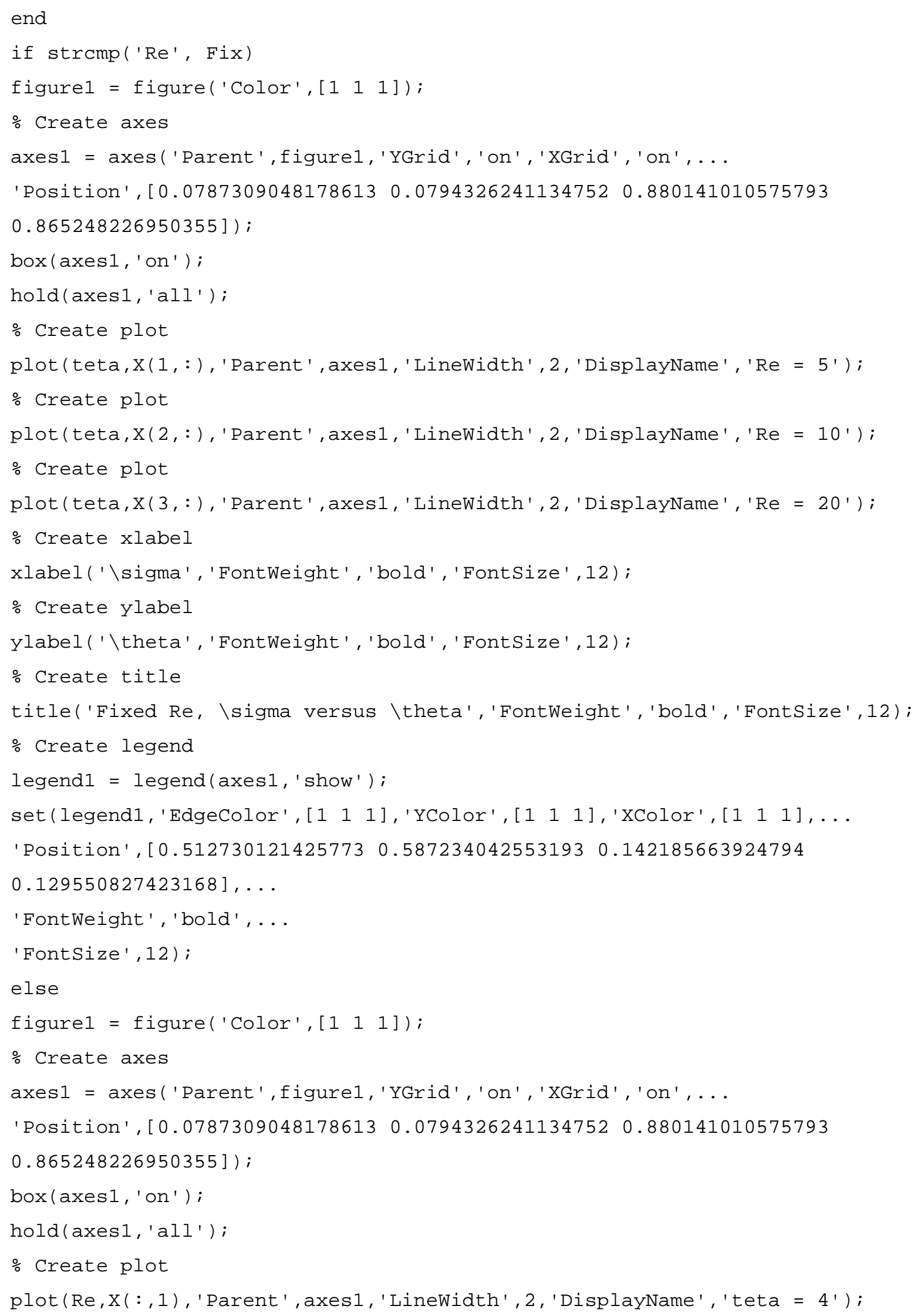


$\%$ create plot

$\operatorname{plot}(\operatorname{Re}, x(:, 2)$, 'Parent ', axes1, 'Linewidth' , 2, 'DisplayName', 'teta = 6');

$\%$ Create plot

$\operatorname{plot}(\operatorname{Re}, \mathrm{X}(:, 3)$, 'Parent ', axes1, 'Linewidth' , 2, 'DisplayName', 'teta = 8');

$\%$ create plot

plot $(\operatorname{Re}, x(:, 4)$, 'Parent ', axes1, 'LineWidth ', 2, 'DisplayName', ' teta = 10');

$\%$ Create $x l a b e l$

xlabel('\sigma', 'FontWeight ', 'bold' , 'FontSize' , 12);

$\%$ create ylabel

ylabel('Re', 'FontWeight', 'bold', 'FontSize', 12);

$\%$ create title

title('Fixed Teta, \sigma versus Re', 'FontWeight','bold', 'FontSize', 12);

$\%$ create legend

legend1 = legend $($ axes1, 'show');

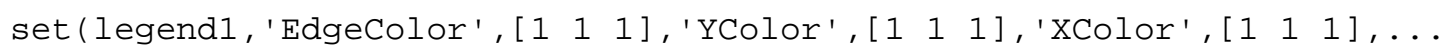

'Position', [0.512730121425773 $\odot .587234042553193 \quad 0.142185663924794$

$0.129550827423168], \ldots$

'FontWeight', 'bold', ...

'FontSize', 12);

end

hold on

if $\operatorname{strcmp}\left(' \mathrm{Re}^{\prime}, \mathrm{Fix}\right)$

plot(teta, sigma(1,:), teta, sigma(2,:), teta, sigma(3,:))

hold off

Error $=100 *$ abs $\left(\left(\right.\right.$ sigma' $\left.-X^{\prime}\right) \cdot /$ sigma' $)$;

\% plotting Errors

figure1 = figure ('Color', $\left.\left[\begin{array}{lll}1 & 1 & 1\end{array}\right]\right)$;

$\%$ create axes

axes1 = axes ('Parent ', figure1, 'YMinorTick' , 'on' ' 'XMinorTick' ', 'on ', . . .

'Position', [0.109612141652614 $\odot .117962466487936 \quad \odot .851602023608769$

$0.807037533512064])$;

$\%$ Uncomment the following line to preserve the $\mathrm{X}$-limits of the axes

$\% \times \lim \left(\operatorname{axes1},\left[\begin{array}{ll}3 & 11\end{array}\right]\right)$;

$\%$ Uncomment the following line to preserve the $\mathrm{Y}$-limits of the axes

$\% y \lim \left(\operatorname{axes1,}\left[\begin{array}{ll}0 & 105\end{array}\right]\right)$;

hold(axes1, 'all');

\% Create multiple lines using matrix input to plot

plot1 = plot (Error, teta, 'Parent', axes1, 'Linewidth', 2); 


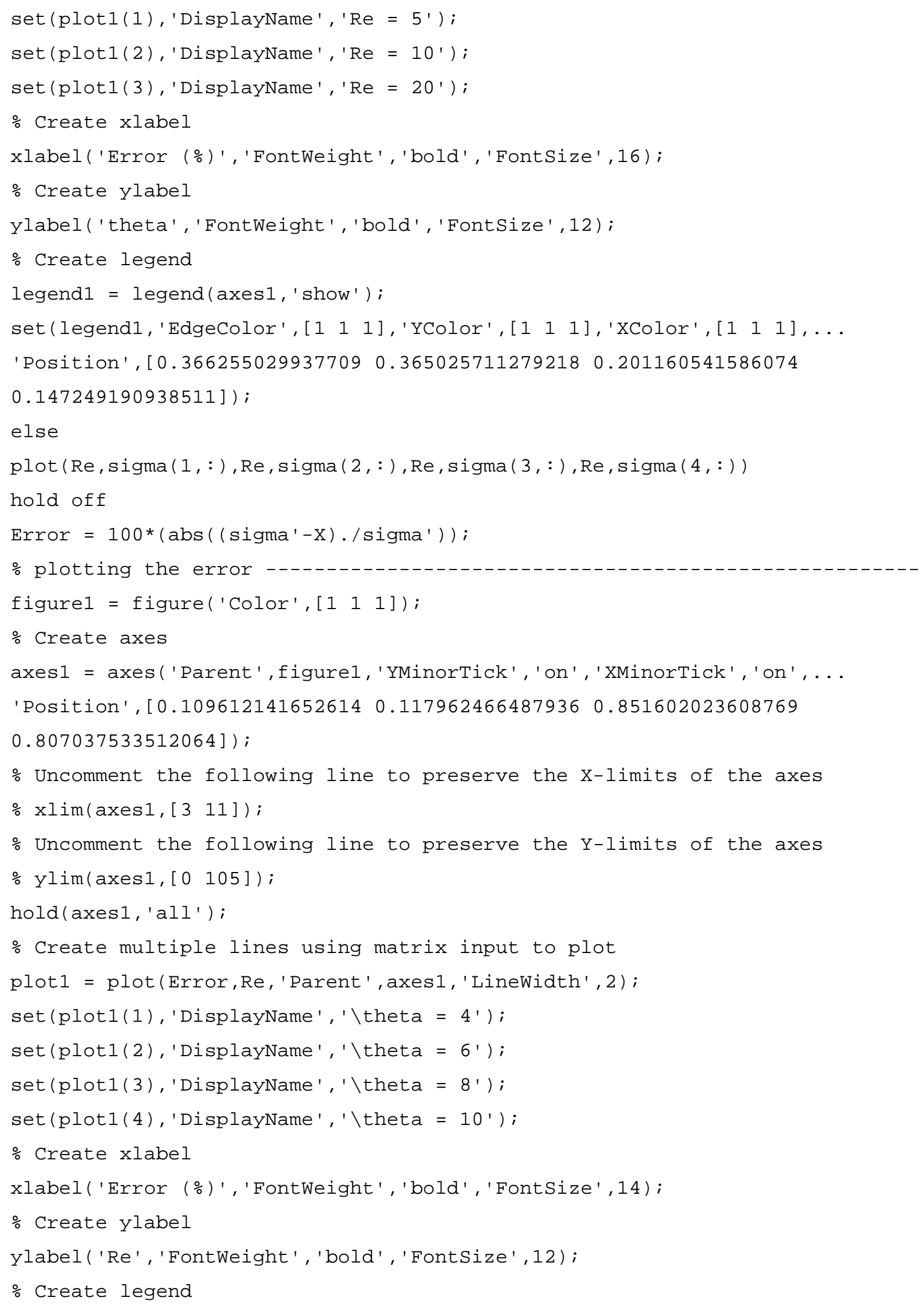


legend1 = legend (axes1, 'show');

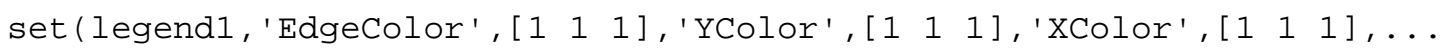

'Position', [0.366255029937709 $\odot .365025711279218 \quad 0.201160541586074$

$0.147249190938511])$;

End

Semi-analytical” Matlab code developed for the axisymmetric theory.

\%\%\%\%\%\%\%\%\%\%\%\%\%\%\%\%\%\%\%\%\%\%\%\%\%\%\%\%\%\%\%\%\%\%\%\%\%\%\%\%\%\%\%\%\%\%\%\%\%\%\%\%\%\%\%\%

\% Task3 Matlab Code \%

\% Adviser: Dr. Slava Akkerman \%

\% Student: Berk Demirgok \%

$\% \%$

$\% \%$

$\% \%$

$\% \%$

\%\%\%\%\%\%\%\%\%\%\%\%\%\%\%\%\%\%\%\%\%\%\%\%\%\%\%\%\%\%\%\%\%\%\%\%\%\%\%\%\%\%\%\%\%\%\%\%\%\%\%\%\%\%\%\%

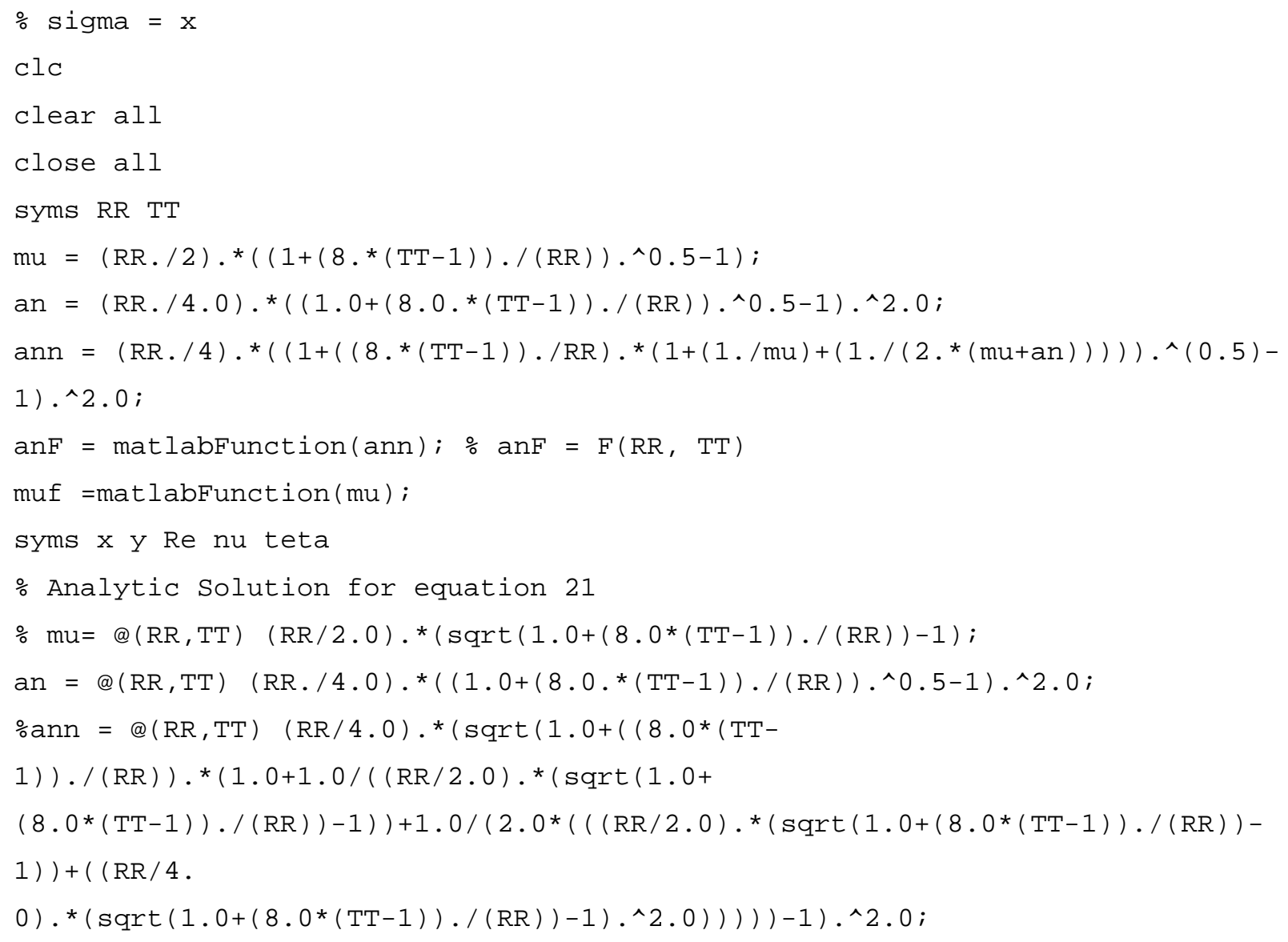




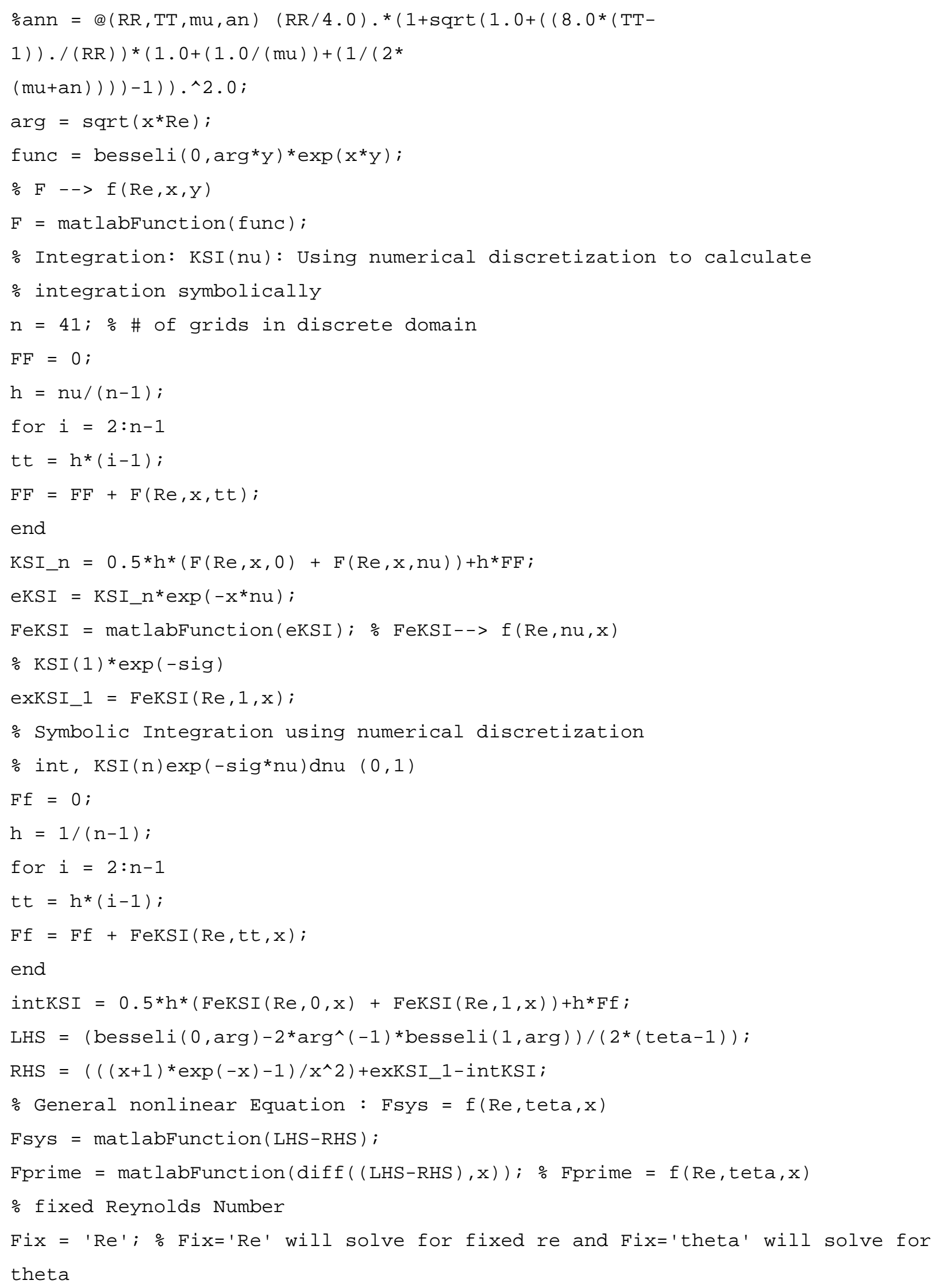




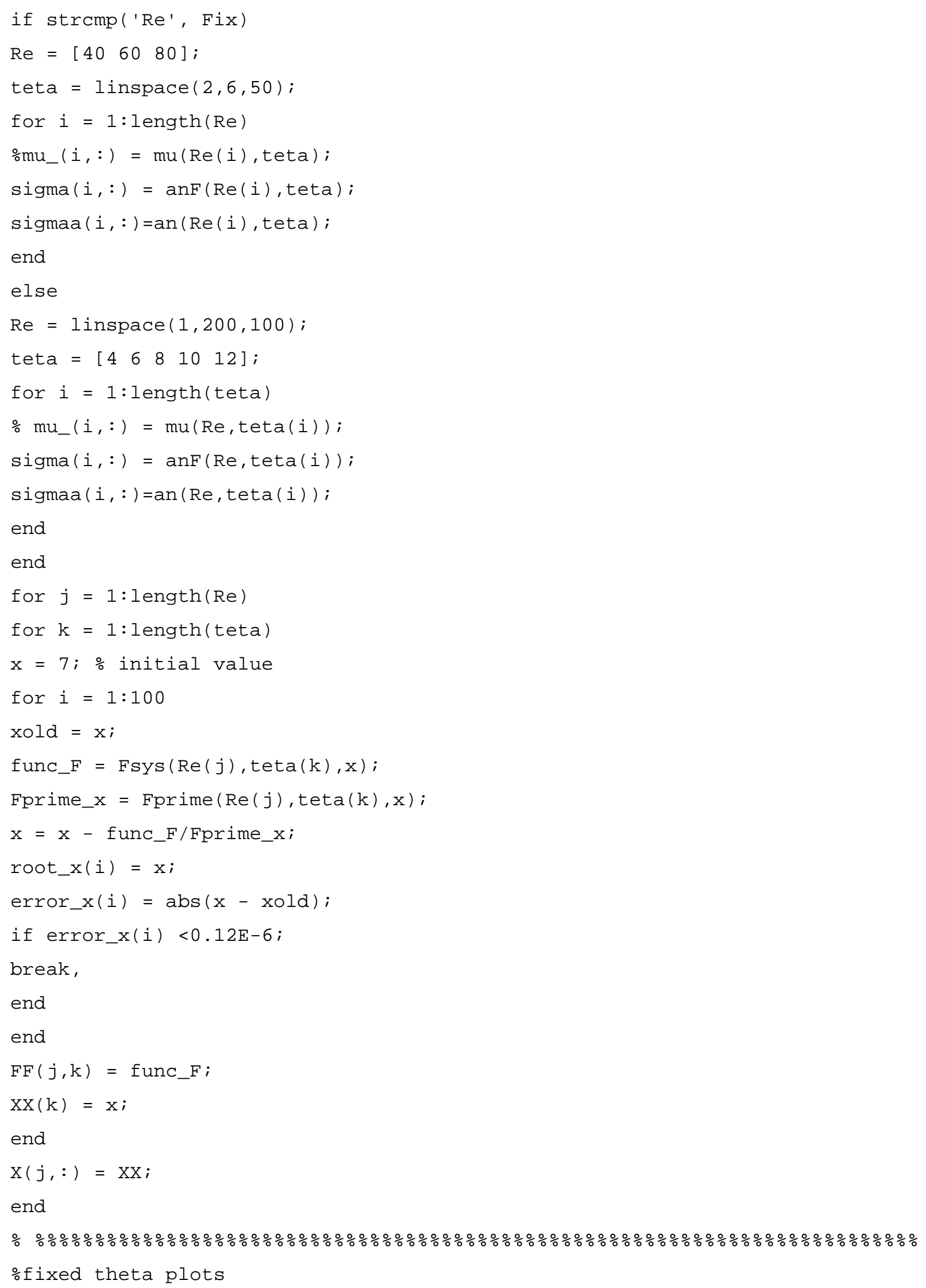




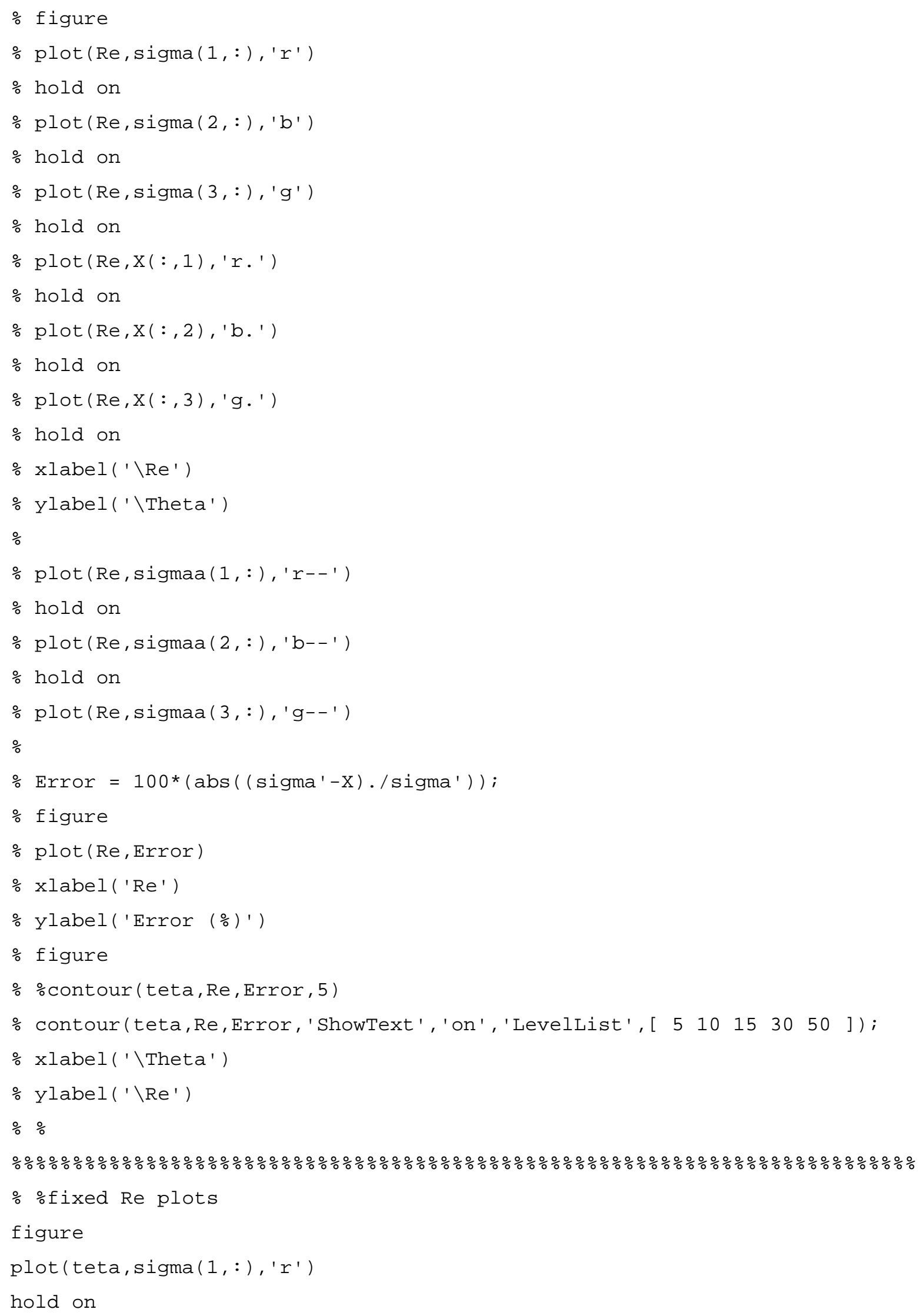




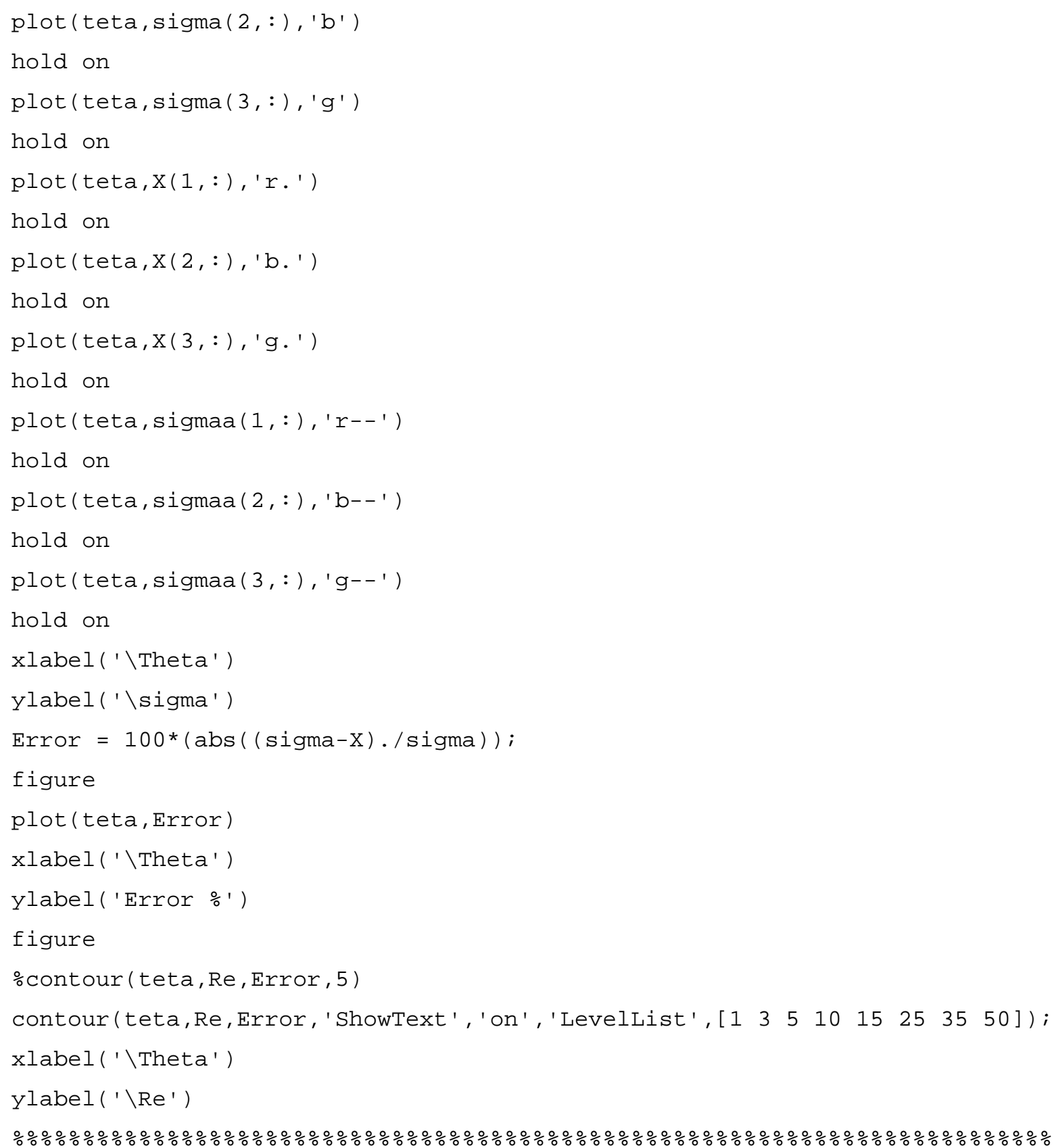




\section{APPENDIX B:}

Here it is demonstrated an illustration sample of the initial file before we start our simulations. Key parameters are explained by short comments.

\begin{tabular}{|c|c|c|}
\hline INITIAL_FRONT_POS & 4.000000 & Position/or radius of initial \\
\hline RADIUS_OF_TUBE & 20.000000 & Radius of tube in $\mathrm{L}_{\mathrm{f}}$ \\
\hline STEP_INCREASE & 1.050000 & Step increase from flame uniform domain \\
\hline WAVE_STEP & 1.250000 & Determines the resolution for wave domain $\left(\mathrm{in}_{\mathrm{f}}\right)$ \\
\hline WAVE_DOMAIN & 1000 & \\
\hline OUTER_DOMAIN_SIZE & 85 & \\
\hline I_CELLS & 10066 & \\
\hline J_CELLS & 81 & \\
\hline REGSTEP & 0.250000 & Grid tapping in flame uniform domain (in $\mathrm{L}_{\mathrm{f}}$ ) \\
\hline UNIFORM_MARGIN & 401 & \\
\hline PRESSURE_FUEL & 100000.000000 & in SI units \\
\hline TEMPERATURE_FUEL & 300.000000 & in SI units \\
\hline EXPANSION_COEFFICINET & 9.000000 & non dimensional thermal expansion coefficient \\
\hline VISCOSITY & 0.000017 & \\
\hline PRANDTL_NUMBER & 1.000000 & non dimensional \\
\hline LEWIS_NUMMBER & 1.000000 & non dimensional \\
\hline GAMMA & 1.400000 & non dimensional \\
\hline TAU_REACTION & \multicolumn{2}{|c|}{$3.983574597289894 \mathrm{e}-08$} \\
\hline MACH_NUMBER & 0.001010 & non dimensional \\
\hline MOLĀ̄_MASS_FUEL & 0.029000 & non dimensional \\
\hline MOLAR_MASS_BURNT & 0.029000 & non dimensional \\
\hline R_UNIV & 8.314340 & non dimensional \\
\hline CV_FUEL & 716.753448 & non dimensional \\
\hline CV_BURNT & 716.753448 & non dimensional \\
\hline E_FUEL & 1505182.241379 & non dimensional \\
\hline E_BURNT & 0.000000 & non dimensional \\
\hline E_ACTIVATION & 59863.248000 & non dimensional \\
\hline DENSITY_FUEL & 1.162650 & non dimensional \\
\hline FLAME_VELOCITY & 0.350478 & in SI units \\
\hline FLAME_THICKNESS & 4.171949526182 & in SI units \\
\hline NUMBËR_OF_SPECIES & 2 & \\
\hline LAMBDA & 2988.169192 & \\
\hline EPS_ACTIVATION & 4.000000 & \\
\hline NSTEP & 1000 & \\
\hline INIT_OUTPUT & 0 & \\
\hline NUMBER_OF_OUTPUT & 10000 & \\
\hline PCOEFF & 0.000000 & \\
\hline REACTION_ORDER & 1.000000 & \\
\hline
\end{tabular}




\section{APPENDIX C:}

Following (Akkerman et al., 2006; Bychkov et al., 2010), a laminar flame front propagating from the closed end of a semi-infinite cylinder of radius $R$ with non-slip wall is considered; see schematic in Fig. 1.3. The formulation is also relevant to the situation of two fronts propagating outwardly in a symmetric manner from an ignition point in the center of a tube with both ends open (Wu and Wang, 2011). The same scaled variables as in Chapter 2 are used, namely, $\eta=r / R, \xi=z / R, \mathbf{w}=\mathbf{u} / U_{f}, \Omega_{w}=U_{w} / U_{f}, \tau=t U_{f} / R$. Then the Navier-Stokes equation ahead of the flame reads

$$
\frac{\partial w_{a}}{\partial \tau}=\Pi(\tau)+\frac{1}{\operatorname{Re}} \frac{1}{\eta} \frac{\partial}{\partial \eta}\left(\eta \frac{\partial w_{a}}{\partial \eta}\right),
$$

where the term $\Pi=-\rho^{-1} \partial P / \partial \xi$ is produced by the flame front propagating with the scaled velocity $\Omega_{w}$ with respect to the fuel mixture. The subscript “a” designates the flow ahead of the flame front. The flame position is described by $\xi_{f}(\eta, \tau)=\xi_{\text {tip }}(\tau)-f(\eta, \tau)$, where $\xi_{\text {tip }} \equiv \xi_{f}(0, \tau)$ designates the flame tip, at the channel axis, and $f(\eta, \tau)$ describes the flame shape, with $f(0, \tau)=0$ by definition. The local burning rate is proportional to the local increase in the flame

surface area, $\sqrt{1+(\partial f / \partial \eta)^{2}}$. In addition, the flame is drifted by the flow. Consequently, with $(\partial f / \partial \eta)^{2}>>1$ everywhere except for the flat region close to the tube axis, the flame evolution and shape are described by

$$
\partial \xi_{f} / \partial \tau-w_{a}=\sqrt{1+(\partial f / \partial \eta)^{2}} \approx \partial f / \partial \eta, \quad \partial f / \partial \tau+\partial f / \partial \eta=w_{a}(0, \tau)-w_{a}(\eta, \tau)
$$


It is also defined that the spatially averaged flame position $\Sigma=\left\langle\xi_{f}\right\rangle$, which is related to the total flame velocity in the laboratory reference frame $U_{L}$ as $\dot{\Sigma}=U_{L} / U_{f}$. Thus averaging Eq. (A.2) yields

$$
\dot{\Sigma}=\langle w\rangle_{a}+\Omega_{w}=\langle w\rangle_{a}+\int_{0}^{1} \frac{\partial f}{\partial \eta} 2 \eta d \eta=\langle w\rangle_{a}+2\left[f(1, \tau)-\int_{0}^{1} f(\eta, \tau) d \eta\right] .
$$

Finally, similar to the analysis for the 2D planar geometry (Akkerman et al., 2006), the instantaneous average velocity of the flow generated by an accelerating flame front in a cylindrical tube is given by

$$
\langle w\rangle_{a}=\frac{\Theta-1}{\Theta}\left[\dot{\Sigma}-(\gamma-1) M_{0} \frac{\Theta-\tilde{m}}{\Theta^{2}}(\dot{\Sigma})^{2}-\frac{M_{0}}{\Theta} \ddot{\Sigma} \Sigma\right],
$$

where $M_{0} \equiv U_{f} / c_{0}<<1$ is the initial Mach number, with the initial sound speed in the fuel mixture being $c_{0}=c\left(T_{0}\right)$, the initial expansion factor $\Theta \equiv \rho_{0} / \rho_{b 0}$, and $\tilde{m} \equiv m_{u} / m_{b}$, where $m_{u}$ and $m_{b}$ are the average molecular weights of the unburned and burned gases, respectively. For a perfect gas one has

$$
\Theta \equiv \rho_{0} / \rho_{b 0}=\left(m_{u} / m_{b}\right)\left(T_{b 0} / T_{0}\right)=1+\tilde{m} Q / C_{P} T_{0},
$$

with the energy release in the reaction $Q$ and the heat capacity at constant pressure $C_{P}$. As such, within the $1^{\text {st }}$-order approximation in $M_{0}<<1$, Eqs. (A.1) - (A.4) describe the flame acceleration and flame-generated flow in a semi-infinite tube with non-slip, adiabatic wall. In the limit of an incompressible flow, $M_{0}=0$, we have $\langle w\rangle_{a}=(\Theta-1) \Omega_{w}, \dot{\Sigma}=\Theta \Omega_{w}$.

Within the $1^{\text {st }}$-order accuracy for $M_{0}<<1$, the solution to the set of Eqs. (A.1) - (A.4) yields the flow velocity profile, 


$$
\begin{gathered}
w_{a}(\eta, \tau) \approx(\Theta-1) \Omega_{s s} \frac{\mu}{\mu-2}\left(1-\frac{\exp [\mu(\eta-1)]}{\sqrt{\eta}}\right) \exp (\sigma \tau)- \\
M_{0} A(\Theta-1)^{2} \Omega_{s s}^{2} \frac{\mu}{\mu-\sqrt{2}}\left(1-\frac{\exp [\sqrt{2} \mu(\eta-1)]}{\sqrt{\eta}}\right) \exp (2 \sigma \tau),
\end{gathered}
$$

with the average velocity

$$
\langle w\rangle_{a}=(\Theta-1) \Omega_{s s} \exp (\sigma \tau)-M_{0} A(\Theta-1)^{2} \Omega_{s s}^{2} \exp (2 \sigma \tau) .
$$

The flame shape is described as

$$
\begin{aligned}
& f(\eta, \tau) \approx(\Theta-1) \Omega_{s s} \frac{\mu}{\mu-2}\left\{\frac{\exp [\mu(\eta-1)]}{(\mu+\sigma) \sqrt{\eta}}-\frac{\sqrt{2 \pi \mu \eta}}{\sigma} \exp (-\mu)[1-\exp (-\sigma \eta)]\right\} \exp (\sigma \tau)- \\
& M_{0} A \frac{(\Theta-1)^{2} \Omega_{s s}^{2} \mu}{\mu-\sqrt{2}}\left\{\frac{\exp [\sqrt{2} \mu(\eta-1)]}{(\sqrt{2} \mu+2 \sigma) \sqrt{\eta}}-\frac{1}{\sigma} \sqrt{\frac{\pi \mu \eta}{2}} \exp (-\sqrt{2} \mu)[1-\exp (-2 \sigma \eta)]\right\} \exp (2 \sigma \tau),
\end{aligned}
$$

such that the total burning rate is given by

$$
\Omega_{w}=2\left[f(1, \tau)-\int_{0}^{1} f(\eta, \tau) d \eta\right]=\Omega_{s s} \exp (\sigma \tau)-M_{0}(\Theta-1)^{2} \Omega_{s s}^{2} A B \exp (2 \sigma \tau),
$$

where

$$
B=[\sigma+(\mu / \sqrt{2})]^{-1}, \quad A=[(\gamma-1)(\Theta-\tilde{m})+\Theta]\left(\frac{1}{\Theta-1}-B\right) .
$$

Consequently, the total flame speed with respect to the laboratory reference frame read

$$
\dot{\Sigma}=\langle w\rangle_{a}+\Omega_{w}=\Theta \Omega_{s s} \exp (\sigma \tau)-M_{0}(\Theta-1)^{2} \Omega_{s S}^{2} A(1+B) \exp (2 \sigma \tau),
$$

with the average flame position,

$$
\Sigma=\frac{\Theta}{\sigma} \Omega_{s s} \exp (\sigma \tau)-M_{0} \frac{(\Theta-1)^{2}}{2 \sigma} \Omega_{s s}^{2} A(1+B) \exp (2 \sigma \tau),
$$

and its acceleration,

$$
\ddot{\Sigma}=\Theta \sigma \Omega_{s s} \exp (\sigma \tau)-2 M_{0} \sigma(\Theta-1)^{2} \Omega_{s s}^{2} A(1+B) \exp (2 \sigma \tau)
$$

Then the flame tip position is given by 


$$
\xi_{\text {tip }}=\Sigma+\langle f\rangle=\left(\frac{\Theta}{\sigma}+\frac{1}{\mu}\right) \Omega_{\text {ss }} \exp (\sigma \tau)-M_{0}(\Theta-1)^{2} \Omega_{s s}^{2} A\left(\frac{1+B}{2 \sigma}+\frac{B}{\sqrt{2} \mu}\right) \exp (2 \sigma \tau),
$$

and the flame tip velocity in the laboratory reference frame

$$
\begin{aligned}
\dot{\xi}_{\text {tip }} \equiv \frac{U_{\text {tip }}}{S_{L}}=\dot{\Sigma}+\langle\dot{f}\rangle & =\left(\Theta+\frac{\sigma}{\mu}\right) \Omega_{\text {ss }} \exp (\sigma \tau)- \\
& M_{0}(\Theta-1)^{2} \Omega_{s s}^{2} A\left[1+B\left(1+\sqrt{2} \frac{\sigma}{\mu}\right)\right] \exp (2 \sigma \tau) .
\end{aligned}
$$

In the $0^{\text {th }}$ order approximation in $M_{0}$, Eqs. (A.6) - (A.15) reproduce the incompressible theory of flame acceleration in cylindrical tubes (Akkerman et al., 2006), while the $1^{\text {st }}$ order corrections elucidate the moderation of the acceleration due to compressibility. It is also noted that Eqs. (A.7), (A.9) - (A.15) agree with the respective formulas obtained in the 2D geometry (Bychkov et al., 2010). However, even in terms of $\mu$ and $\sigma$, the factor $B$, Eq. (A.8), is twice the respective 2D factor (Bychkov et al., 2010). Furthermore, in the axisymmetric case, the factor $\mu$ is about twice that of the $2 \mathrm{D}$ case for the same Re. Consequently, the axisymmetric acceleration exponent $\sigma$ is about 4 times larger than the planar one, yielding considerably stronger flame acceleration, as well as the stronger relative effect of the moderation, in the cylindrical, axisymmetric geometry.

The moderating flame acceleration can be further demonstrated in the form of a differential equation for the average flame position. In the $0^{\text {th }}$ order approximation of $M_{0}$, the accelerating flame dynamics is determined by the differential equation

$$
\ddot{\Sigma}=\sigma \dot{\Sigma},
$$

which can be extended in the $1^{\text {st }}$ order approximation as

$$
\ddot{\Sigma}=\sigma \dot{\Sigma}\left(1-C_{1} \dot{\Sigma}\right)
$$


with $C_{1}=M_{0}\left(1-\Theta^{-1}\right)^{2} A(1+B)$. Integration of Eq. (A.17) with the initial condition

$$
\dot{\Sigma}(0)=\Theta \Omega_{s s}-M_{0}(\Theta-1)^{2} \Omega_{s s}^{2} A(1+B),
$$

yields the average flame front velocity in the laboratory frame and the average flame position,

$$
\dot{\Sigma}=\frac{\Theta \Omega_{s s} \exp (\sigma \tau)}{1+M_{0} C_{1} \Theta \Omega_{s s} \exp (\sigma \tau)}, \quad \Sigma=\frac{1}{\sigma M_{0} C_{1}} \ln \left[1+M_{0} C_{1} \Theta \Omega_{s s} \exp (\sigma \tau)\right] .
$$

The total burning rate and the flame tip evolution can be estimated in the similar manner. For the burning rate, the $0^{\text {th }}$ order relation, $\dot{\Omega}_{w}=\sigma \Omega_{w}$, extends to

$$
\dot{\Omega}_{w}=\sigma \Omega_{w}\left(1-\tilde{C} \Omega_{w}\right),
$$

with $\tilde{C}=M_{0}(\Theta-1)^{2} A B$, initial condition $\Omega_{w}(0)=\Omega_{s s}-M_{0}(\Theta-1)^{2} \Omega_{s s}^{2} A B$, and the solution

$$
\Omega_{w}=\frac{\Omega_{s s} \exp (\sigma \tau)}{1+\widetilde{C} \Omega_{s s} \exp (\sigma \tau)} .
$$

Similarly, the evolution of the flame tip is described by

$$
\begin{aligned}
& \ddot{\xi}_{\text {tip }}=\sigma \dot{\xi}_{\text {tip }}\left(1-C_{2} \dot{\xi}_{\text {tip }}\right), \\
& \dot{\xi}_{\text {tip }}=\frac{(\Theta+\sigma / \mu) \Omega_{s s} \exp (\sigma \tau)}{1+C_{2}(\Theta+\sigma / \mu) \Omega_{s s} \exp (\sigma \tau)}, \\
& \xi_{\text {tip }}=\frac{1}{\sigma C_{2}} \ln \left[1+C_{2}(\Theta+\sigma / \mu) \Omega_{s s} \exp (\sigma \tau)\right],
\end{aligned}
$$

where

$$
C_{2}=M_{0} \frac{(\Theta-1) A}{(\Theta+\sigma / \mu)^{2}}\left(\frac{1+B}{2 \sigma}+\frac{B}{\sqrt{2} \mu}\right) \approx M_{0} A\left(\frac{\Theta-1}{\Theta}\right)^{2} .
$$

Here we account for the effect of compressibility in the $1^{\text {st }}$ order approximation for the initial Mach number related to flame propagation, $M_{0} \equiv U_{f} / c_{0}<<1$. In order to describe a compression wave, we shall neglect the multi-dimensional flame structure approximating the flame front as a one-dimensional flat discontinuity surface in the locus $\Sigma=\Sigma(t)$. In the limit of 
weak compression, the isentropic approximation may be adopted. Then, the scaled density $\tilde{\rho}=\rho / \rho_{0}$, pressure $\tilde{p}=P / P_{0}$ and temperature $\tilde{T}=T / T_{0}$ just ahead of the flame are given by :

$$
\begin{aligned}
& \tilde{\rho}_{a}=\left(1+\frac{\gamma-1}{2} M_{0}\langle w\rangle_{a}\right)^{\frac{2}{\gamma-1}} \approx 1+M_{0}\langle w\rangle_{a}, \\
& \tilde{p}_{a}=\left(1+\frac{\gamma-1}{2} M_{0}\langle w\rangle_{a}\right)^{\frac{2 \gamma}{\gamma-1}} \approx 1+\gamma M_{0}\langle w\rangle_{a}, \\
& \tilde{T}_{a}=\left(1+\frac{\gamma-1}{2} M_{0}\langle w\rangle_{a}\right)^{2} \approx 1+(\gamma-1) M_{0}\langle w\rangle_{a},
\end{aligned}
$$

Matching conditions at the flame front take the form

$$
\begin{aligned}
& \tilde{\rho}_{a}\left(\dot{\Sigma}-\langle w\rangle_{a}\right)=\tilde{\rho}_{b f}\left(\dot{\Sigma}-\langle w\rangle_{b f}\right), \\
& \tilde{p}_{a}+\gamma M_{0}^{2} \tilde{\rho}_{a}\left(\dot{\Sigma}-\langle w\rangle_{a}\right)^{2}=\tilde{p}_{b f}+\gamma M_{0}^{2} \tilde{\rho}_{b f}\left(\dot{\Sigma}-\langle w\rangle_{b f}\right)^{2}, \\
& \tilde{T}_{a}+\varepsilon-1+\frac{\gamma-1}{2} M_{0}^{2}\left(\dot{\Sigma}-\langle w\rangle_{a}\right)^{2}=\tilde{T}_{b f}+\frac{\gamma-1}{2} M_{0}^{2}\left(\dot{\Sigma}-\langle w\rangle_{b f}\right)^{2},
\end{aligned}
$$

where the index "bf" denotes the burnt matter just behind the flame front and $\varepsilon$ stands for the initial temperature jump across the front, with $\varepsilon=\Theta / \tilde{m}$ in the perfect gas model, where $\tilde{m}$ is the ratio of the average molecular weights of unburned and burned gases, $\tilde{m}=m_{u} / m_{b}$. In the $1^{\text {st }}$ order approximation of $M_{0}$, Eqs. (A.30), (A.31) and the perfect gas law yield

$$
\tilde{p}_{b f}=\tilde{p}_{a}, \quad \tilde{T}_{b f}=\widetilde{T}_{a}+\varepsilon-1, \quad \tilde{\rho}_{b f}=\tilde{\rho}_{a} / \theta,
$$

with the instantanoues expansion factor accounting for compressibility being

$$
\theta \equiv \tilde{m} \tilde{T}_{b f} / \tilde{T}_{a}=\tilde{m}\left\{1+(\varepsilon-1) / T_{a}\right\} .
$$

Obviously, $\theta=\Theta$ when compression is negligible, $M_{0}\langle w\rangle_{a} \rightarrow 0$. But, as soon as the flame accelerates, $\tilde{T}_{a}$ increases and $\theta$ reduces, thereby moderating the flame acceleration. Let us next consider the flow in the burnt gas. From the continuity equation 


$$
\frac{\partial \tilde{\rho}_{b}}{\partial \tau}+\frac{\partial}{\partial \xi}\left(\tilde{\rho}_{b} w_{b}\right)=0
$$

and the condition of adiabatic compression, $p \propto \rho^{\gamma}$, we find

$$
\frac{\partial w_{b}}{\partial \xi}=-\frac{1}{\tilde{\rho}_{b}} \frac{d \tilde{\rho}_{b}}{d \tau}=-\frac{1}{\gamma \tilde{p}_{b}} \frac{d \tilde{p}_{b}}{d \tau},
$$

where the substantial derivative is taken for a particular gas parcel. For the same 1D model, we have one more equation for pressure, namely

$$
M_{0}^{2}\left(\frac{\partial w_{b}}{\partial \tau}+w_{b} \frac{\partial w_{b}}{\partial \xi}\right)=-\frac{1}{\tilde{\rho}_{b}} \frac{\partial \tilde{p}_{b}}{\partial \xi}
$$

Neglecting the second order term of $M_{0}$ in Eq. (A.35), we obtain uniform pressure distribution in the burnt gas, so pressure is a function of time only, $\tilde{p}_{b}=\tilde{p}_{b f}(\tau)=\tilde{p}_{a}(\tau)$. Then

$$
w_{b}=-\frac{1}{\gamma \tilde{p}_{a}} \frac{d \tilde{p}_{a}}{d \tau} \xi, \quad w_{b f}=-\frac{1}{\gamma \tilde{p}_{a}} \frac{d \tilde{p}_{a}}{d \tau} \Sigma .
$$

Finally, substituting Eqs. (A.27), (A.28), (A.32) and (A.35) into Eq. (A.29), within the $1^{\text {st }}$ order approximation of $M_{0}$, we find

$$
\frac{1}{\tilde{m}}\left(1+\frac{\varepsilon-1}{\tilde{T}_{a}}\right)\left(\dot{\Sigma}-\langle w\rangle_{a}\right)=\dot{\Sigma}+\frac{M_{0}}{1+\gamma M_{0}\langle w\rangle_{a}} \frac{d\langle w\rangle_{a}}{d \tau} \Sigma,
$$

with the final result

$$
\begin{gathered}
\langle w\rangle_{a} \approx\left[\frac{\Theta-1}{\Theta}-\frac{\varepsilon-1}{\Theta \varepsilon}(\gamma-1) M_{0}\langle w\rangle_{a}\right] \dot{\Sigma}-\frac{M_{0}}{\Theta}\left[1+\frac{\varepsilon-1}{\varepsilon}(\gamma-1) M_{0}\langle w\rangle_{a}\right] \frac{d\langle w\rangle_{a}}{d \tau} \Sigma \\
\approx \frac{\Theta-1}{\Theta}\left[\dot{\Sigma}-(\gamma-1) M_{0} \frac{\Theta-\tilde{m}}{\Theta^{2}}(\dot{\Sigma})^{2}-\frac{M_{0}}{\Theta} \ddot{\Sigma} \Sigma\right] .
\end{gathered}
$$

